\title{
Árvores $k$-restritas e aproximações para o Problema de Steiner em Grafos
}

Eduardo Kazuaki Gondo

\author{
DISSERTAÇÃO APRESENTADA AO \\ INSTITUTO DE MATEMÁTICA E ESTATÍSTICA DA \\ UNIVERSIDADE DE SÃO PAULO \\ PARA OBTENÇÃO DO GRAU DE \\ MESTRE EM \\ CIÊNCIA DA COMPUTAÇÃO
}

Orientadora: Prof ${ }^{a}$. Dr ${ }^{a}$. Cristina Gomes Fernandes

— São Paulo, março de 2002 - 


\title{
Árvores $k$-restritas e aproximações para o Problema de Steiner em Grafos
}

\author{
Este exemplar corresponde à redação final da dissertação, \\ devidamente corrigida, defendida por \\ Eduardo Kazuaki Gondo e aprovada pela comissão julgadora.
}

São Paulo, 10 de maio de 2002.

Banca examinadora:

- Prof ${ }^{a}$. Dr ${ }^{a}$. Cristina Gomes Fernandes (orientadora) - IME-USP

- Prof. Dr. Carlos Eduardo Ferreira - IME-USP

- Prof. Dr. Flávio Keidi Miyazawa - IC-UNICAMP 
Aos meus pais e

ao meu irmão, 


\section{Agradecimentos}

Os principais objetivos deste trabalho foram aprender todos os passos da pesquisa científica ou, como gosto de dizer, "aprender a aprender" e conseguir expressar as idéias e resultados dessa pesquisa de forma clara e precisa. Porém os primeiros passos de qualquer aprendizado não é feito solitariamente, é preciso a ajuda de várias pessoas. Para essas pessoas vão meus sinceros agradecimentos.

À minha orientadora, Professora Cristina Gomes Fernandes, pela excelente orientação, pelos ensinamentos, constante presença, dedicação, atenção, paciência, estímulo e amizade.

Aos meus pais e ao meu irmão pelo apoio, estímulo e amor.

Ao meu amigo Carlos que me incentivou ao ingresso do mestrado e pela sua compreensão nesta "reta final".

Aos professores, Ivan de Camargo, José Augusto Soares e Stavros Christodoulou por acreditarem na realização deste mestrado.

Aos Professores Paulo Feofiloff e Carlos Eduardo Ferreira pelas suas dicas no início deste trabalho.

Aos meus amigos do Dieese pelo estímulo e companheirismo.

Aos meus amigos do IME pelos momentos de descontração.

À Simone pela compreensão, paciência e estímulo. 


\title{
Resumo
}

O Problema de Steiner em Grafos consiste em encontrar, em um grafo com custos nas arestas, uma árvore de custo mínimo contendo um dado conjunto de vértices.

Esse problema tem um papel central na área de algoritmos de aproximação e diversas de suas variantes têm aplicações, por exemplo, em projeto de redes de comunicação.

Neste trabalho apresentamos um estudo de vários algoritmos de aproximação para este problema. Os algoritmos escolhidos baseiam-se na utilização de árvores k-restritas e formam uma série, cada um deles tendo explorado melhor uma idéia introduzida pelo anterior. Todos eles foram projetados durante a última década, sendo o mais recente, o melhor algoritmo de aproximação para o problema até o momento em termos de razão de aproximação.

\begin{abstract}
The Steiner Tree Problem consists in finding a minimum cost tree that spans a given subset of vertices. This problem plays a central role in approximation algorithms. Many of its variants have applications on network design.

In this work we present a study of several approximation algorithms for the Steiner Tree Problem. The selected algorithms are based on the use of k-restricted trees and form a series, each one exploring further an idea introduced by the previous one. All of them were developed during the last decade and the last of them has the best performance ratio known for the problem so far.
\end{abstract}




\section{Sumário}

Introdução 2

1 Definiçōes específicas e notação 5

1.1 Teoria dos grafos . . . . . . . . . . . . . . . . . 5

1.2 Problema de Steiner em Grafos . . . . . . . . . . . . . . . . . . 12

2 Algoritmo das árvores 3-restritas 20

2.1 Descrição do algoritmo . . . . . . . . . . . . . . . . . . . 20

2.2 Análise do algoritmo das árvores 3-restritas . . . . . . . . . . . . . . . 21

2.3 Algoritmo original de Zelikovsky . . . . . . . . . . . . . . . . . . . . . . 29

3 Algoritmo de Berman e Ramayer $\quad 31$

3.1 Descrição do algoritmo . . . . . . . . . . . . . . . . . . . . . 31

3.2 Análise do algoritmo de Berman e Ramayer . . . . . . . . . . . . . . . . 37

3.3 Comparação com o algoritmo das árvores 3 - restritas . . . . . . . . . . . . 39

4 Algoritmo do ganho relativo $\quad 40$

4.1 Ganho relativo . . . . . . . . . . . . . . . . . . . 40

4.2 Descrição do algoritmo . . . . . . . . . . . . . . . . . . . 40

4.3 Análise do algoritmo do ganho relativo . . . . . . . . . . . . . . . . 42

5 Algoritmo do pré-processamento $\quad 45$

5.1 Floresta de terminais e ganho ponderado . . . . . . . . . . . . . . 45

5.2 Descrição do algoritmo . . . . . . . . . . . . . . . . . . . . 47

5.3 Análise do algoritmo do pré-processamento . . . . . . . . . . . . . . . 49

6 Algoritmo de Robins e Zelikovsky 54

6.1 Contração de florestas de terminais e ganho relativo à perda . . . . . . . . 54

6.2 Descrição do algoritmo . . . . . . . . . . . . . . . . . . . 57

6.3 Análise do algoritmo de Robins e Zelikovsky . . . . . . . . . . . . . 58

$\begin{array}{ll}\text { Considerações finais } & 64\end{array}$ 


\section{Introdução}

Problemas de otimização são de interesse nas mais diversas áreas do conhecimento. Chamamos de Otimização Combinatória a área da Ciência da Computação que estuda esses tipos de problemas. Um problema de otimização combinatória pode ser definido basicamente da seguinte forma: dada uma função definida sobre um conjunto finito $A$, encontre, se existir, um elemento de A que minimize (maximize) a função dada.

Embora a definição seja simples, para muitos desses problemas não é fácil encontrar um elemento de $A$ que responda a pergunta do problema. Em geral, a dificuldade está no fato que $A$, mesmo sendo finito, pode ter um número muito grande de elementos. Isso torna inviável, do ponto de vista computacional, gerar e verificar todos os elementos de $A$ para encontrar um melhor. Vários desses problemas fazem parte do conjunto dos problemas $\mathcal{N} \mathcal{P}$-difíceis. Isso significa que, para vários desses problemas, não conhecemos e nem mesmo esperamos encontrar algoritmos eficientes para resolvê-los. Contudo não podemos simplesmente deixar de estudar tais problemas, porque eles possuem importantes aplicações práticas.

Cormen, Leiserson e Rivest [10] sugerem duas abordagens para problemas $\mathcal{N} \mathcal{P}$-difíceis:

- Algoritmos que produzam soluções ótimas mas que no pior caso consumam tempo exponencial no tamanho da entrada. (Estes algoritmos podem ser adequados apenas para instâncias pequenas ou particulares.)

- Algoritmos eficientes (isto é, que consomem tempo polinomial no tamanho da entrada) que produzam soluções aproximadas com uma garantia de qualidade, ou seja, um limite para o erro entre o custo da solução produzida pelo algoritmo e o custo de uma solução ótima do problema. Estes algoritmos são denominados algoritmos de aproximação e chamamos razão de aproximação a sua garantia de qualidade. (A definição precisa desses conceitos será dada no capítulo 1.)

O Problema de Steiner em Grafos é um dos exemplos de problema de otimização $\mathcal{N P}$-difícil [18]. Informalmente, ele pode ser assim definido: dado um grafo $G$ com custos não-negativos nas arestas, determinar um subgrafo conexo de $G$ de custo mínimo que contenha um dado conjunto de vértices. Esse problema aparece no contexto de projetos de circuitos VLSI [29], árvores filogenéticas [28], problemas de conexão [23], etc.

O surgimento do Problema de Steiner data do século XVII. Segundo Maculan [35], a primeira formulação do problema foi feita pelo matemático francês Pierre de Fermat. O 
problema era o seguinte: dadas três cidades, deseja-se construir uma rede de estradas de comprimento total minimo de forma que se possa ir de qualquer uma das três cidades a qualquer outra através dessas estradas.

Utilizando geometria, podemos-reescrever formalmente o problema acima: dados três pontos no plano euclidiano, determinar um conjunto de segmentos de reta que interligue estes pontos de tal forma que a soma dos comprimentos dos segmentos de reta seja mínima.

Ainda de acordo com Maculan, no século XIX, o geômetra alemão Jacob Steiner reuniu diversas soluções em um trabalho. Por causa deste trabalho, o problema, primeiramente proposto por Fermat, passou a ser conhecido como o Problema de Steiner.

Courant e Robbins [11] apresentaram uma maneira de determinar a solução deste problema através de uma construção utilizando régua e compasso. Sejam $A, B$ e $C$ os três pontos, sendo que $A$ é tal que o ângulo formado pelos segmentos $\overline{A B}$ e $\overline{A C}$ é máximo. Temos dois casos para analisar. Se esse ângulo é maior ou igual a $120^{\circ}$, então a solução é dada pelos segmentos $\overline{A B}$ e $\overline{A C}$. Caso contrário, seja $P$ o ponto do plano euclidiano tal que os segmentos $\overline{A P}, \overline{B P}$ e $\overline{C P}$ formam, dois a dois, um ângulo de $120^{\circ}$. Esses segmentos formam a solução do problema. Vejam as figuras abaixo.

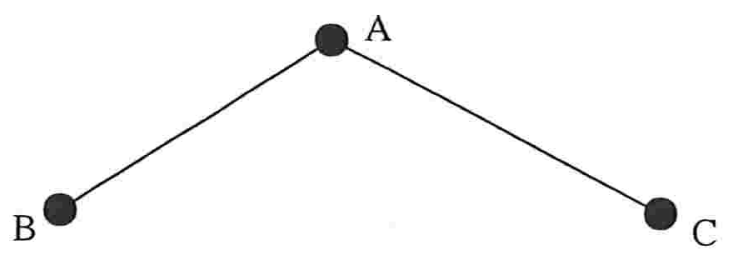

Figura 1: $\hat{\mathrm{A}} \geq 120^{\circ}$

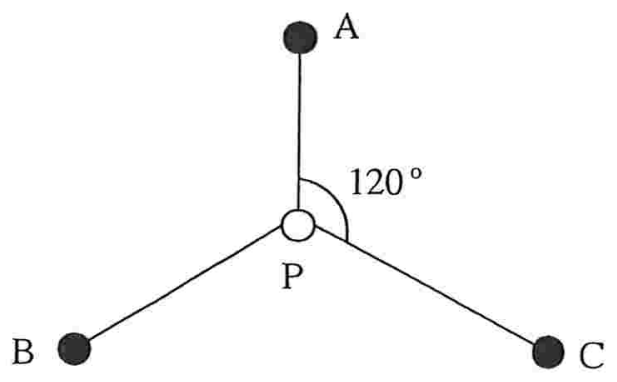

Figura 2: $\hat{\mathrm{A}}<120^{\circ}$

O Problema de Steiner em Grafos (PSG) é uma variante do Problema de Steiner. Neste trabalho, apresentaremos uma resenha dos algoritmos de aproximação para o PSG baseados em árvores $k$-restritas (definidas no capítulo 1). O uso de árvores $k$-restritas mostrou-se eficaz para o PSG - os algoritmos de aproximação com as melhores razões de aproximação conhecidas para o PSG utilizam essas árvores. 
Este trabalho está organizado da seguinte forma. No primeiro capítulo, definimos o Problema de Steiner em Grafos, discutimos a sua complexidade computacional e comentamos algumas técnicas de solução do problema. Além disso, estabelecemos a notação que será usada na apresentação dos algoritmos e apresentamos o primeiro algoritmo de aproximação para o problema - o ingênuo algoritmo da árvore geradora mínima com razão de aproximação 2.

No segundo capítulo, apresentamos o algoritmo das árvores 3-restritas, proposto por Zelikovsky [48] em 1993. Durante vários anos, 2 foi a melhor razão de aproximação constante conhecida para o problema. O algoritmo das árvores 3-restritas foi o primeiro a conseguir uma razão de aproximação constante menor que 2. O algoritmo é simples porém sua análise é complexa e o artigo original continha falhas que foram explicitamente consertadas por Gröpl, Hougardy, Nierhoff e Prömel [21].

No terceiro capítulo, apresentamos o algoritmo de Berman e Ramayer [6], que utiliza árvores $k$-restritas para uma constante $k \geq 3$. No quarto capítulo, descrevemos o algoritmo do ganho relativo, elaborado por Zelikovsky [49], que introduziu uma nova maneira de escolher árvores $k$-restritas. No quinto capítulo, temos o algoritmo do pré-processamento, proposto por Karpinski e Zelikovsky [31], que introduziu o conceito de perda de uma árvore de Steiner.

No sexto capítulo apresentamos o algoritmo de Robins e Zelikovsky [42], proposto em 2000, cuja razão de aproximação é a melhor conhecida para o PSG até o presente. O último capítulo contém algumas considerações finais.

Esses algoritmos evoluiram uns dos outros e mostram diferentes maneiras de se usar árvores $k$-restritas no projeto de algoritmos de aproximação para o PSG. É importante ressaltar que o nosso objetivo principal no estudo desses algoritmos é a demonstração de uma razão de aproximação e não a determinação precisa do tempo de execução desses algoritmos. Esperamos que esta dissertação facilite a compreensão desses interessantes algoritmos e sirva como ponto de partida e de apoio para o estudo do PSG e de problemas correlatos. 


\section{Capítulo 1}

\section{Definições específicas e notação}

Neste capítulo descrevemos alguns dos conceitos utilizados neste trabalho, definimos formalmente o Problema de Steiner em Grafos e discutimos algumas de suas principais características.

\subsection{Teoria dos grafos}

Os conceitos e definições abaixo foram retirados dos livros de Bondy e Murty [8] e de Diestel [12].

Um grafo $G=(V, E)$ consiste de um conjunto finito e não-vazio $V_{G}$ e de um conjunto $E_{G}$ de pares não-ordenados de elementos de $V_{G}$. Os elementos de $V_{G}$ são chamados de vértices de $G$ e os de $E_{G}$ são as arestas de $G$.

Se $e=\{u, v\} \in E_{G}$, então os vértices $u$ e $v$ são os extremos de $e$. Algumas vezes denotamos e simplesmente por $u v$. Dois vértices $u, v$ em $V_{G}$ são adjacentes se existir uma aresta com extremos $u$ e $v$. Duas arestas são paralelas se têm os mesmos extremos. Um laço é uma aresta que tem os extremos iguais.

Um grafo $G$ é simples se ele não tem arestas paralelas e nem laços. Se cada vértice de $G$ é adjacente a todos os outros, então $G$ é completo.

Se $A \subseteq V_{G}$, denotamos por $\delta(A)$ o conjunto de todas as arestas de $G$ que têm um extremo em $A$ e o outro em $V_{G} \backslash A$. Se $u \in A$ e $v \notin A$, dizemos que $\delta(A)$ separa $u$ e $v$. Chamamos tal conjunto de corte de $G$ definido por $A$. Se $A=\{v\}$, escrevemos $\delta(v)$ em vez de $\delta(\{v\})$. O número $|\delta(v)|$ é o grau de $v$.

Se os grafos $H$ e $G$ são tais que $V_{H} \subseteq V_{G}$ e $E_{H} \subseteq E_{G}$, então dizemos que $H$ é um subgrafo de $G$. Se $H$ é um subgrafo de $G$ e $E_{H}$ é o conjunto de todas as arestas de $G$ que têm ambos os extremos em $V_{H}$, então $H$ é um subgrafo induzido de $G$. Se $V_{H}=V_{G}$, dizemos que $H$ é um subgrafo gerador de $G$. Se $A$ é um conjunto de vértices de $G$, o subgrafo induzido de $G$ que tem $A$ por conjunto de vértices é denotado por $G[A]$.

Seja $F$ um conjunto de pares não-ordenados de vértices de $G$. Denotamos por $G+F$ o grafo $\left(V_{G}, E_{G} \cup F\right)$. De modo análogo, $G-F$ é o grafo $\left(V_{G}, E_{G} \backslash F\right)$. Se $F=\{f\}$, 
escrevemos $G+f$ e $G-f$, respectivamente, para $G+\{f\}$ e $G-\{f\}$.

Um passeio é uma seqüência $C=\left(v_{0}, e_{1}, v_{1}, e_{2}, v_{2}, \ldots, e_{k}, v_{k}\right)$ de elementos de um grafo $G$ onde $v_{0}, \ldots, v_{k}$ são vértices e cada $e_{i}$ é uma aresta com extremos $v_{i-1}$ e $v_{i}$. Os vértices $v_{0}$ e $v_{k}$ são os extremos de $C$. Para simplificar, podemos omitir as arestas $\mathrm{e}$ representar um passeio por sua seqüência de vértices, $\left(v_{0}, \ldots, v_{k}\right)$. Algumas vezes interpretamos $C$ como o conjunto de arestas de $C$. O passeio é fechado se $v_{0}=v_{k}$ e aberto caso contrário. Se as arestas de $C$ são todas distintas, então $C$ é chamado de trilha. Se os vértices de $C$ são todos distintos, então $C$ é chamado de caminho. Se $k \geq 1$ e os vértices $v_{1}, \ldots, v_{k}$ são todos distintos e $v_{0}=v_{k}$, então $C$ é um circuito.

Um grafo euleriano $G$ é um grafo onde o grau de todos os vértices é par. Uma trilha euleriana $P$ em $G$ é um passeio fechado que passa uma única vez em cada aresta de $G$. Um circuito hamiltoniano $C$ em um grafo $G$ é um passeio fechado que passa exatamente uma vez em cada vértice de $G$.

Um grafo $G$ é conexo se, para cada par $u, v$ de vértices, existe um caminho em $G$ com extremos $u$ e $v$. Um componente de $G$ é um subgrafo conexo maximal de $G$. Dizemos que dois vértices $u$ e $v$ de $G$ estão conectados ou que $u$ está conectado a $v$ em $G$ se $u$ e $v$ estão em um mesmo componente de $G$. De modo análogo, dizemos que um conjunto de vértices $X$ de $G$ está conectado, ou $G$ conecta $X$, se todos os vértices de $X$ estão em um mesmo componente de $G$. Uma floresta é um grafo simples sem circuitos. Uma árvore é uma floresta conexa. Algumas vezes confundiremos uma árvore com o seu conjunto de arestas e um componente de um grafo com seu conjunto de vértices. Uma árvore enraizada em $v$ é uma árvore onde todas as arestas da árvore têm uma orientação no sentido do vértice $v$. Esta orientação é obtida através de todos os caminhos em $M$ de um vértice $u$ ao vértice $v$, onde $u \neq v$. O vértice $v$ é denominado raiz da árvore. A figura 1.1 ilustra. algumas das definições acima mencionadas.

Dados um grafo $G$ e uma função $c$ que associa um número a cada aresta de $G$, denotamos por $c(F)$ a soma $\sum_{e \in F} c(e)$ para todo conjunto $F$ de arestas de $G$. Quando a função $c$ está subentendida, chamamos o número $c(F)$ de custo de $F$ e o número $c(e)$ de custo de e. Para um subgrafo $H$ de $G$, escrevemos $c(H)$ em vez de $c\left(E_{H}\right)$ e chamamos este número de custo de $H$.

Se $G$ é completo, dizemos que $c$ satisfaz a desigualdade triangular se

$$
c(i j) \leq c(i k)+c(k j)
$$

para quaisquer três vértices distintos $i, j$ e $k$ de $G$.

Uma árvore geradora mínima de $(G, c)$ é uma árvore geradora em $G$ de custo mínimo. Ou seja, $M^{*}$ é uma árvore geradora mínima se para toda árvore geradora $M$ em $G$ tem-se que $c\left(M^{*}\right) \leq c(M)$. Denotamos o custo de $M^{*}$ por $\operatorname{mst}(G, c)$.

Seja $W$ um conjunto de vértices de $G$. A redução de $c$ em $W$ é a função que coincide com $c$ exceto nas arestas cujas duas extremidade estão em $W$, onde ela vale zero. 


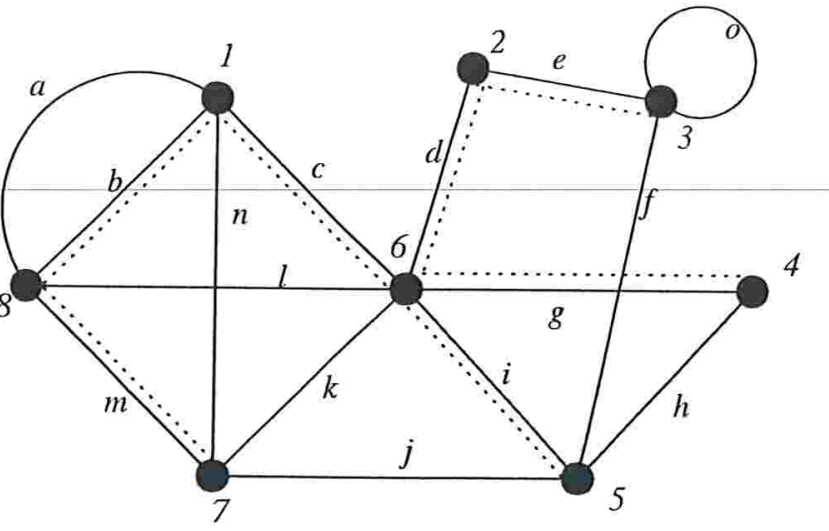

Figura 1.1: Sejam $G=(V, E)$, onde $V:=\{1,2,3,4,5,6,7,8\}$ e $E:=$ $\{a, b, c, d, e, f, g, h, i, j, k, l, m, n, o\}$. A aresta $o$ é um exemplo de laço e as arestas $a$ e $b$ são paralelas. Se $A:=\{4,5\}$, então $\delta(A):=\{f, g, i, j\}$. A seqüência $P:=(1, a, 8, m, 7, k, 6)$ é um exemplo de um caminho em $G$ de 1 a 6 . O grafo $G-\{a, o\}$ é simples e as arestas pontilhadas representam as arestas de uma árvore geradora de $G$.

Denotamos a redução de $c$ em $W$ por $c[W]$. Mais especificamente,

$$
c[W](e)= \begin{cases}c(e) & \text { se } e \notin E_{G[W]}, \\ 0 & \text { se } e \in E_{G[W]} .\end{cases}
$$

A restrição de $c$ a $G[W]$ é a função que coincide com $c$ exceto nas arestas que não estão em $E_{G[W]}$, onde ela não está definida. Mais especificamente, se $\dot{c}$ é a restrição de $c$ a $G[W]$, então

$$
\dot{c}(e)=c(e) \text { para toda aresta } e \text { em } E_{G[W]} .
$$

Algumas vezes usamos $c$ no lugar da restrição de $c$ a $G[W]$.

\section{Árvores de Steiner e árvores $k$-restritas}

Considere um grafo completo $G$, uma função $c$ de $E_{G}$ em $Q_{>0}$ satisfazendo a desigualdade triangular e um conjunto $R$ não-vazio de vértices de $G$. Chamamos os vértices de $R$ de terminais e os de $V_{G} \backslash R$ de vértices de Steiner. Uma árvore $T$ em $G$ é uma árvore de Steiner de $(G, R)$ se $R \subseteq V_{T}$. Uma árvore de Steiner mínima de $(G, c, R)$ é uma árvore de Steiner de $(G, R)$ de custo mínimo, ou seja, $T^{*}$ é uma árvore de Steiner mínima de $(G, c, R)$ se $c\left(T^{*}\right) \leq c(T)$ para toda árvore de Steiner $T$ de $(G, R)$. Denotamos por $\operatorname{smt}(G, c, R)$ o custo de uma árvore de Steiner mínima de $(G, c, R)$. 
Uma k-árvore de $(G, R)$ é uma árvore em $G$ com $k$ folhas cujos vértices internos são de Steiner (ou seja, não estão em $R$ ) e as folhas são terminais (estão em $R$ ). Para uma $k$ árvore $T_{k}$ de $(G, R)$, denotamos por $V T\left(T_{k}\right)$ o conjunto dos terminais de $T_{k}$ e por $V S\left(T_{k}\right)$ o conjunto dos vértices de Steiner de $T_{k}$.

Seja $T$ uma árvore de Steiner de $(G, R)$. Se $T^{\prime}$ é uma subárvore maximal de $T$ que é uma $i$-árvore de $(G, R)$ para algum $i$, dizemos que $T^{\prime}$ é um componente cheio de $T$. Se, para algum $k$, todo componente cheio de $T$ é uma $i$-árvore em $(G, R)$ com $i \leq k$, dizemos que $T$ é uma árvore $k$-restrita de $(G, R)$.

Uma árvore $k$-restrita minima de $(G, c, R)$ é uma árvore $k$-restrita de $(G, R)$ de custo mínimo, ou seja, $T^{*}$ é uma árvore $k$-restrita mínima de $(G, c, R)$ se $c\left(T^{*}\right) \leq c(T)$ para toda árvore $k$-restrita $T$ de $(G, R)$. Denotamos por $\operatorname{smt}_{k}(G, c, R)$ o custo de uma árvore $k$-restrita mínima de $(G, c, R)$. A figura 1.2 ilustra as definições acima.

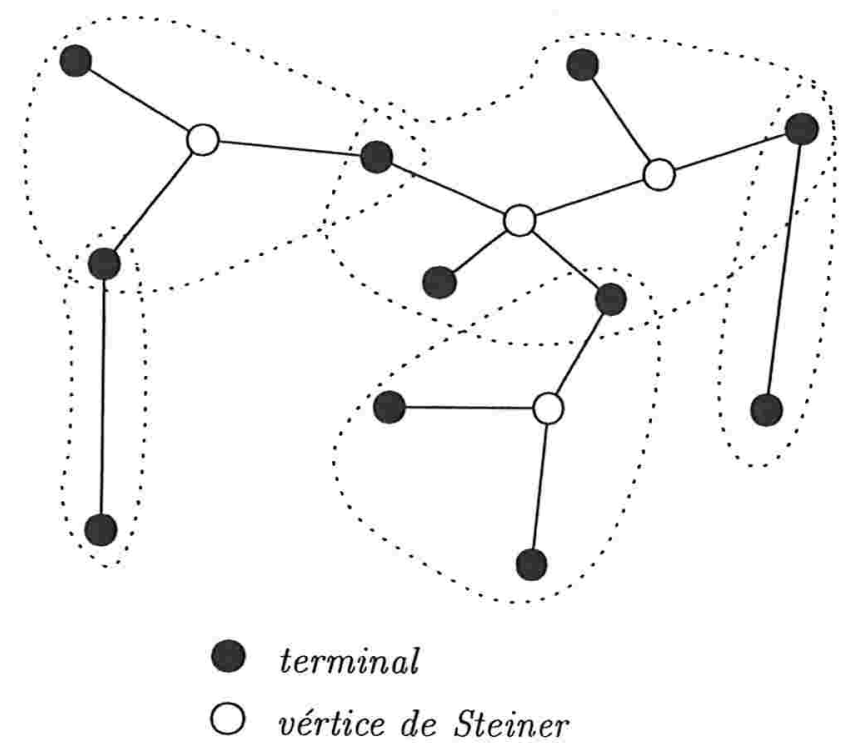

Figura 1.2: Exemplo de árvore de Steiner. Cada componente cheio está destacado pelas linhas pontilhadas. Note que, nesse exemplo, o número máximo de terminais de um componente cheio é 5. Portanto trata-se de uma árvore 5-restrita.

Note que, se uma árvore de Steiner mínima $T$ de $(G, c, R)$ é uma árvore $k$-restrita, então $T$ é uma árvore $k$-restrita mínima. Entretanto, o inverso não é verdadeiro. Por exemplo, uma árvore 2-restrita mínima, que é uma árvore geradora mínima no subgrafo induzido pelos terminais, nem sempre é uma árvore de Steiner mínima. Veja a figura 1.3.

\section{Conjunto remoção e função projeção}

Sejam $H$ um grafo e $\dot{c}$ uma função de $E_{H}$ em $Q_{>0}$. Considere uma árvore geradora mínima $M$ de $(H, \dot{c})$ e um conjunto $\tau$ de vértices de $H$. Um separador de $\tau$ em $M$ é 


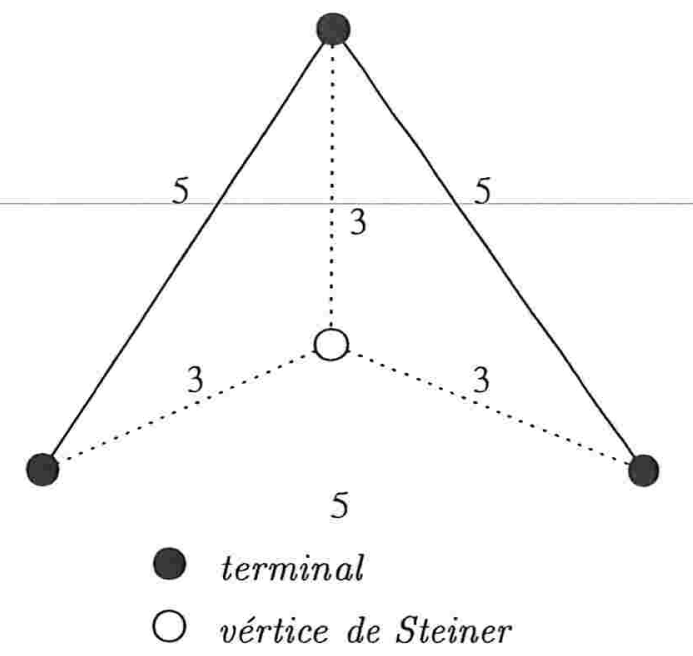

Figura 1.3: No grafo as linhas normais são arestas de uma árvore geradora mínima sobre os terminais e as linhas pontilhadas são as aresta de uma árvore de Steiner mínima.

um conjunto minimal de aresta de $M$ cuja remoção desconecta todo par de vértices de $\tau$. Um conjunto remoção $D$ de $\tau$ em $M$ é um separador de $\tau$ em $M$ com $\dot{c}(D)$ máximo. Denotamos por ind $(M, \dot{c}, \tau)$ o número $\dot{c}(D)$, onde $D$ é um conjunto remoção de $\tau$ em $M$.

Se $\tau=\{x, y\}$, então $\operatorname{ind}(M, \dot{c}, \tau)$ é o custo de uma aresta de custo máximo no caminho em $M$ entre os extremos de $x y$ e é denotado por $i n d(M, \dot{c}, x y)$. Neste caso, chamamos uma tal aresta de indice de $x y$ em $M$. Note que $\dot{c}(x y) \geq i n d(M, \dot{c}, x y)$ para qualquer aresta $x y$, porque, se $\dot{c}(x y)<i n d(M, \dot{c}, x y)$, então uma aresta de custo máximo no caminho em $M$ entre $x$ e $y$ poderia ser substituída pela aresta $x y$ resultando em uma árvore geradora $M^{\prime}$ de $(H, \dot{c})$ tal que $\dot{c}\left(M^{\prime}\right)<\dot{c}(M)$. Um absurdo já que $M$ é uma árvore geradora mínima de $(H, \dot{c})$.

$\mathrm{O}$ custo de um conjunto remoção de $\tau$ em $M$ também pode ser definido a partir da redução de $\dot{c}$ em $\tau$. Veja a seguinte expressão,

$$
\operatorname{ind}(M, \dot{c}, \tau)=\operatorname{mst}(H, \dot{c})-\operatorname{mst}(H, \dot{c}[\tau])
$$

Isso, em particular, mostra que a função índice na verdade não depende da árvore geradora mínima $M$. Porém, como em geral associamos o valor do índice ao custo de um conjunto remoção, é conveniente manter $M$ como parâmetro.

Seja $D$ um conjunto remoção $\tau$ em $M$. A função projeção $p$ de $D$ em $\tau$ relativa a $M$ é uma função que associa cada aresta de $D$ uma aresta de $H$, definida do seguinte modo. Para cada aresta $e$ de $D$, existem dois componentes de $M-D$, digamos $X_{e}$ e $Y_{e}$, cada um contendo um dos extremos de $e$. Sejam $x$ e $y$ os vértices de $\tau$ em $X_{e}$ e $Y_{e}$ respectivamente (cada componente de $M-D$ tem exatamente um vértice de $\tau$, pois $D$ é minimal). A função projeção associa à aresta $e$ a aresta $x y$. Observe que a imagem da função projeção 
é uma árvore geradora de $H[\tau]$. Veja na figura 1.4 um conjunto remoção $D$ de $\tau$ em $M$ e a imagem da função projeção $p$ de $D$ em $\tau$ relativa a $M$.

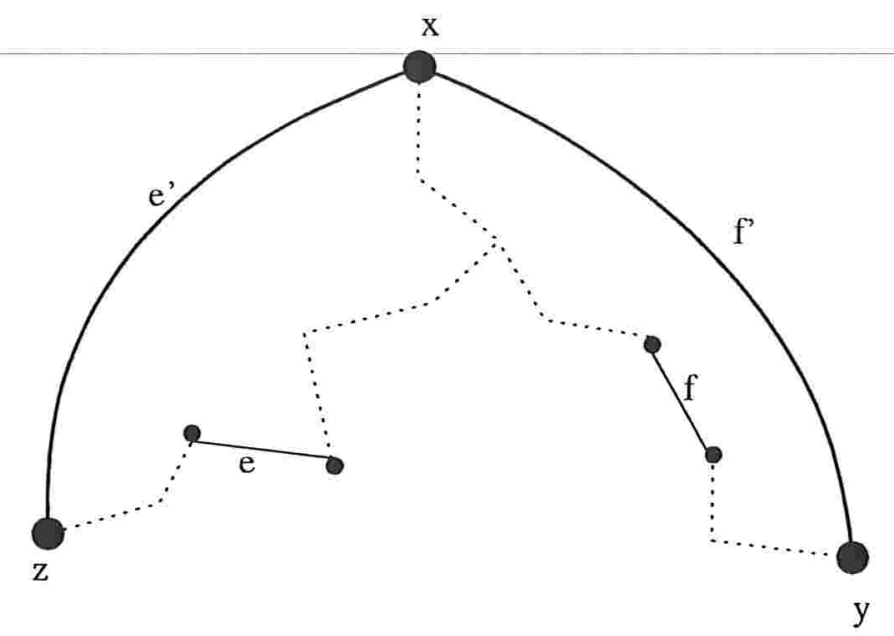

Figura 1.4: $O$ conjunto remoção $D:=\{e, f\}$ e a imagem da função projeção $p(D):=$ $\left\{e^{\prime}, f^{\prime}\right\}$ sobre $\tau:=\{x, y, z\}$ em $M$, onde $M$ são as arestas pontilhadas mais as arestas de $D$.

Vejamos algumas propriedades sobre o custo de um conjunto remoção.

Lema 1.1 Sejam $H$ um grafo, $\dot{c}$ uma função de $E_{H}$ em $Q_{>0}$ e $M$ uma árvore geradora mínima de $(H, \dot{c})$. Para todo conjunto $\tau$ de vértices de $H$ com $|\tau| \geq 3$, temos que

$$
\operatorname{ind}(M, \dot{c}, \tau) \leq \operatorname{ind}(M, \dot{c}, \tau \backslash\{v\})+\operatorname{ind}(M, \dot{c}, u v),
$$

para qualquer $v$ e $u$ em $\tau$, onde $u \neq v$. Além disso, a igualdade vale para algum $u$ em $\tau$, $u \neq v$.

Prova. Seja $D$ o conjunto remoção de $\tau$ em $M$. Em $M-D$ existem $|\tau|$ componentes, cada um deles contendo um vértice de $\tau$. Seja $A$ o componente que contém $v$. Seja $e$ uma aresta de custo mínimo em $D$ que conecta $A$ a um outro componente, digamos $B$. Note que $D \backslash\{e\}$ é um conjunto remoção de $\tau \backslash\{v\}$ em $M$. Assim, temos a seguinte igualdade

$$
\operatorname{ind}(M, \dot{c}, \tau)=\operatorname{ind}(M, \dot{c}, \tau \backslash\{v\})+\dot{c}(e) .
$$

Veja a figura 1.5. Note que, pela escolha de $e$, temos que $\dot{c}(e) \leq \operatorname{ind}(M, \dot{c}, u v)$ para todo $u$ em $\tau \backslash\{v\}$ (e a igualdade vale quando $u$ é o vértice de $\tau$ em $B$ ). De fato, $\dot{c}(e) \leq \dot{c}(f)$ onde $f$ é a aresta em $D \cap \delta(A)$ que separa $u$ de $v$, e portanto $\dot{c}(e) \leq \operatorname{ind}(M, \dot{c}, u v)$. Portanto

$$
\operatorname{ind}(M, \dot{c}, \tau) \leq \operatorname{ind}(M, \dot{c}, \tau \backslash\{v\})+\operatorname{ind}(M, \dot{c}, u v),
$$




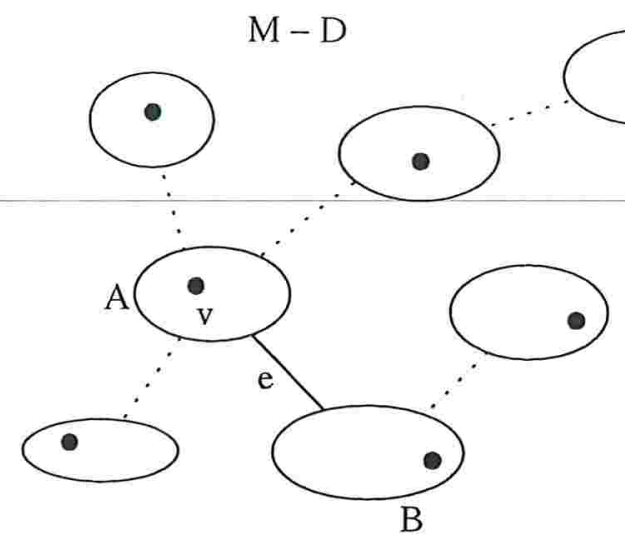

- Vértices de $\tau$

Figura 1.5: As elipses são os componentes de $M-D$ e as linhas pontilhadas são as arestas do conjunto $D$.

valendo a igualdade para pelo menos um $u$ em $\tau \backslash\{v\}$.

O lema e o corolário abaixo serão de grande importância na determinação de uma razão de aproximação dos algoritmos que veremos.

Lema 1.2 Sejam $\tau_{0}$ e $\tau_{1}$ conjuntos de vértices de $H$ com pelo menos dois terminais, $\dot{c}$ uma função de $E_{H}$ em $Q_{>0}, M$ uma árvore geradora mínima de $(H, \dot{c})$ e $D$ um conjunto remoção de $\tau_{0}$ em $M$ e sua respectiva função projeção $p$ relativa a $M$. Seja $\dot{c}^{\prime}$ uma função tal que, para toda aresta e de $H$, tem-se que $\dot{c}^{\prime}(e)=\dot{c}(e)$ se e $\notin p(D)$ e $\dot{c}^{\prime}(p(e)) \leq$ ind $(M, \dot{c}, e)$ se $e \in D$. Se $M^{\prime}:=M-D+p(D)$ então

$$
\operatorname{ind}\left(M, \dot{c}, \tau_{1}\right) \geq \operatorname{ind}\left(M^{\prime}, \dot{c}^{\prime}, \tau_{1}\right) \text {. }
$$

Prova. A prova é por indução em $\left|\tau_{1}\right|$. Primeiramente vamos mostrar que, se $\left|\tau_{1}\right|=2$, então o lema vale. Sejam $x$ e $y$ os elementos em $\tau_{1}$.

Sejam $P$ o caminho em $M$ de $x$ a $y$ e $P^{\prime}$ o caminho em $M^{\prime}$ de $x$ a $y$. Se $P \cap D=\emptyset$, então $P=P^{\prime}$ e não há nada a provar. Se $P \cap D \neq \emptyset$, sejam $e_{1}, \ldots, e_{k}$ as arestas de $P \cap D$ e $f_{j}:=p\left(e_{j}\right)$ para $j=1, \ldots, k$. Seja $C_{j}$ o único circuïto em $M+f_{j}$.

É fácil ver que $f_{j} \in P^{\prime}$ para todo $j$ e que $P^{\prime}$ consiste de trechos de $P$ e dos $C_{j}$, para $j=1, \ldots, k$. Seja $f$ uma aresta de custo máximo em $P^{\prime}$, ou seja, $f$ é tal que $\dot{c}^{\prime}(f)=\operatorname{ind}\left(M^{\prime}, \dot{c}^{\prime}, x y\right)$. Se $f \in P$, então ind $\left(M^{\prime}, \dot{c}^{\prime}, x y\right)=\dot{c}^{\prime}(f)=\dot{c}(f) \leq \operatorname{ind}(M, \dot{c}, x y)$. Se $f \notin P$, então $f \in C_{j}$ para algum $j$ e ind $\left(M^{\prime}, \dot{c}^{\prime}, x y\right)=\dot{c}^{\prime}(f) \leq \dot{c}\left(e_{j}\right) \leq \operatorname{ind}(M, \dot{c}, x y)$.

Agora, suponha que $\left|\tau_{1}\right|>2$. Pelo lema 1.1, existem $u$ e $v$ em $\tau_{1}$ onde $u \neq v$ tal que

$$
\operatorname{ind}\left(M, \dot{c}, \tau_{1}\right)=\operatorname{ind}\left(M, \dot{c}, \tau_{1} \backslash\{v\}\right)+\operatorname{ind}(M, \dot{c}, u v) \text {. }
$$


Pela hipótese de indução quando aplicada no lado direito da igualdade acima, temos que

$$
\begin{aligned}
\operatorname{ind}\left(M, \dot{c}, \tau_{1}\right) & \geq \operatorname{ind}\left(M^{\prime}, \dot{c}^{\prime}, \tau_{1} \backslash\{v\}\right)+\operatorname{ind}\left(M^{\prime}, \dot{c}, u v\right) \\
& \geq \operatorname{ind}\left(M^{\prime}, \dot{c}^{\prime}, \tau_{1}\right)
\end{aligned}
$$

onde a última desigualdade vale pelo lema 1.1.

Corolário 1.3 Sejam $\tau_{0}$ e $\tau_{1}$ conjuntos não-vazios de vértices de $H, \dot{c}$ uma função de $E_{H}$ em $Q_{>0}$ satisfazendo a desigualdade triangular e $M$ uma árvore geradora minima de $(H, \dot{c})$. Se $M^{\prime}$ é uma árvore geradora mínima de $\left(H, \dot{c}\left[\tau_{0}\right]\right)$ então

$$
\operatorname{ind}\left(M, \dot{c}, \tau_{1}\right) \geq \operatorname{ind}\left(M^{\prime}, \dot{c}\left[\tau_{0}\right], \tau_{1}\right) .
$$

\section{Ganho}

Considere novamente um grafo completo $G$, uma função $c$ de $E_{G}$ em $Q_{>0}$ satisfazendo a desigualdade triangular e um conjunto $R$ não-vazio de vértices de $G$. Seja $T_{\tau}^{*}$ uma árvore de Steiner mínima de $(G, c, \tau)$, onde $\tau$ é um subconjunto de $R, \dot{c}$ é a restrição de $c$ a $G[R]$ e $M$ uma árvore geradora mínima de $(G[R], \dot{c})$. O ganho de $\tau$ em $M$ indica se é ou não vantajoso utilizar $T_{\tau}^{*}$ para compor com $M$ uma árvore de Steiner de custo baixo. Matematicamente, o ganho de $\tau$ em $M$ é o número $g(M, \tau):=\operatorname{ind}(M, \dot{c}, \tau)-\operatorname{smt}(G, c, \tau)$. (Fica subentendida a dependência do ganho de $G$ das funções $c$ e $\dot{c}$.) Na figura 1.6 mostramos o ganho de $\tau$ em $M$.

Lema 1.4 Sejam $G$ um grafo completo, $c$ uma função de $E_{G}$ em $Q_{>0}$ satisfazendo a desigualdade triangular, $R$ um conjunto não-vazio de vértices de $G$ e $\tau$ um subconjunto de $R$. Considere também $\dot{c}$ e $\dot{c}^{\prime}$ funções de $E_{G}[R]$ em $Q_{>0}, M$ e $M^{\prime}$ árvores geradoras minimas de $(G[R], \dot{c})$ e de $\left(G[R], \dot{c}^{\prime}\right)$ respectivamente. Se ind $(M, \dot{c}, \tau) \geq \operatorname{ind}\left(M^{\prime}, \dot{c}^{\prime}, \tau\right)$, então

$$
g(M, \tau) \geq g\left(M^{\prime}, \tau\right) .
$$

Prova. Segue diretamente da definição de ganho.

\subsection{Problema de Steiner em Grafos}

Nesta seção, apresentamos o Problema de Steiner em Grafos, partindo de sua definição até o primeiro algoritmo de aproximação conhecido para o problema. Grande parte das informações desta seção foi retirada da dissertação de mestrado de Ferreira [15]. 


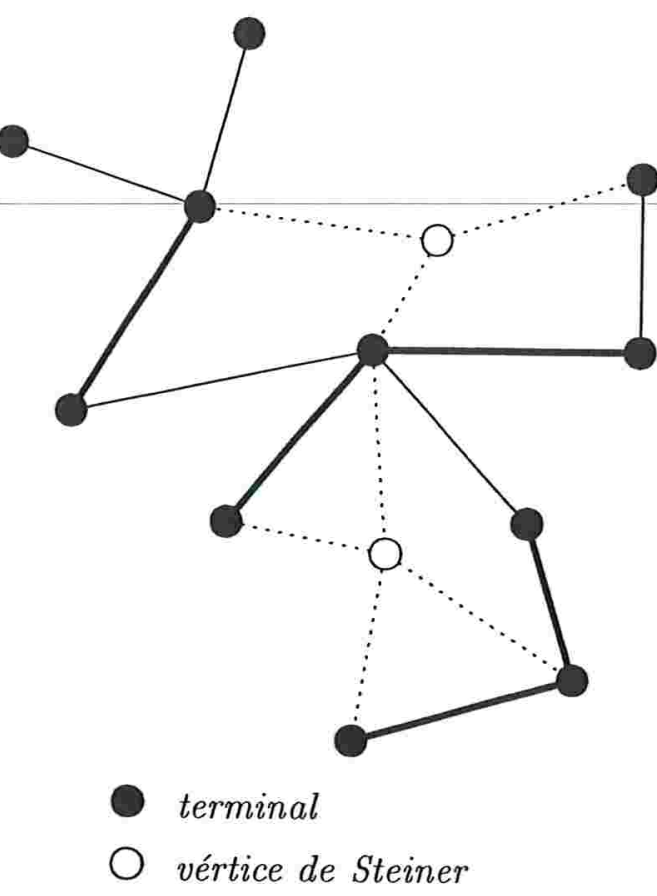

Figura 1.6: As linhas pontilhadas são as arestas de uma árvore de Steiner mínima $T^{*}$, as linhas finas juntamente com as grossas representam as arestas de uma árvore geradora $M$ e as linhas grossas representam um conjunto remoção $D$ de $V T\left(T^{*}\right)$ em $M$. O ganho de $V T\left(T^{*}\right)$ em $M$ é o custo de $D$ menos o custo de $T^{*}$.

\section{Definição}

Considere um grafo $G=(V, E)$ conexo, uma função $c$ de $E_{G}$ em $Q_{>0}$ e um conjunto $R$ não-vazio de vértices de $G$ - os terminais. O objetivo do Problema de Steiner em Grafos é encontrar uma árvore de Steiner mínima de $(G, c, R)$.

A primeira versão do Problema de Steiner apareceu como um problema de geometria [35]. A formulação utilizando os elementos da Teoria dos Grafos apareceu, pela primeira vez, em 1971. Ela foi proposta por Hakimi [24] e por Dreyfus e Wagner [14].

Algumas observações podem ser feitas sobre as instâncias do PSG. Se existir em $G$ arestas paralelas, podemos eliminá-las deixando apenas uma aresta de menor custo dentre as arestas paralelas. Laços também podem ser eliminados do grafo. Vértices de Steiner de grau 1 junto com a aresta incidente a este vértice podem ser eliminados.

O fecho métrico de $(G, c)$ é o par $(\bar{G}, \bar{c})$, onde $\bar{G}$ é um grafo completo com conjunto de vértices $V_{G}$ e, para qualquer par de vértices $u$ e $v$ de $V_{G}, \bar{c}(u v)$ é o custo de um caminho de $u$ até $v$ de custo mínimo de $(G, c)$, ou seja

$$
\bar{c}(u v):=\min \{c(P): P \text { é um caminho de } u \text { até } v \text { em } G\} \text {. }
$$


Note que, a função $\bar{c}$ satisfaz a desigualdade triangular. Além disso, se $\bar{T}$ é uma árvore de Steiner em $(\bar{G}, \bar{c}, R)$ e $T$ é uma árvore de Steiner de $(G, c, R)$ obtida através da substituição de cada aresta $u v$ de $\bar{T}$ pelas arestas de um caminho mínimo de $u$ a $v$ em $G$ e da remoção de uma aresta de cada eventual circuito criado a partir desta substituição, então $c(T) \leq c(\bar{T})$.

Com base nessas observações, podemos nos restringir a instâncias do PSG que consistam de um grafo completo e uma função custo nas arestas satisfazendo a desigualdade triangular.

\section{Complexidade computacional}

Nesta subseção comentaremos sobre a complexidade computacional do PSG e de algumas de suas variantes. Os conceitos sobre complexidade computacional utilizados nessa dissertação podem ser encontrados em [17, 38].

Segundo Karp [30], através do problema da cobertura exata consegue-se mostrar que o problema de decisão associado ao PSG é $\mathcal{N} \mathcal{P}$-completo portanto o PSG é $\mathcal{N} \mathcal{P}$-difícil.

Se $|R|=2$, o PSG é equivalente a encontrar um caminho de custo mínimo entre dois vértices num grafo com custos não-negativos nas arestas. Neste caso, existem algoritmos eficientes (veja, por exemplo [13]). Se $R=V_{G}$, então o PSG é equivalente a encontrar uma árvore geradora mínima de $(G, c)$. Os dois algoritmos mais conhecidos para encontrar uma árvore geradora mínima em um grafo são os algoritmos de Kruskal [33] e o de Prim [40]. Logo, podemos assumir no restante deste trabalho que $2<|R|<\left|V_{G}\right|$.

\section{Técnicas de solução}

Apesar do PSG ser $\mathcal{N} \mathcal{P}$-difícil, muitos algoritmos foram projetados para tentar encontrar uma solução ótima. Nesta subseção, comentaremos de forma bem sucinta alguns desses algoritmos e as técnicas envolvidas.

Um dos primeiros algoritmos para resolver o PSG foi elaborado por Hakimi [24]. Ele utilizou o que denominamos de enumeração explícita. Esta técnica se baseia em construir todas as árvores de Steiner e escolher uma de menor custo. A complexidade deste algoritmo é polinomial no número de terminais e exponencial no número de vértices de Steiner. Lawler [34], valendo-se do fato das propriedades do fecho métrico, apresentou um algoritmo cuja complexidade é polinomial no número de vértices de Steiner e exponencial no número de terminais. É importante notar que, se o número de terminais ou o número de vértices de Steiner está fixo, então os algoritmos acima resolvem o PSG em tempo polinomial.

Dreyfus e Wagner [14] utilizaram programação dinâmica para resolver o PSG. Shore, Foulds e Gibbons [43] também projetaram um algoritmo que resolve o PSG. Eles usaram busca exaustiva (branch and bound) na árvore de soluções.

Uma outra abordagem para resolver o PSG é formulá-lo como um problema de programação inteira $[1,3,4,35,37]$. Ferreira [15] mostra o resultado da aplicação de ferramentas 
da teoria dos poliedros ao PSG.

Uma abordagem que tem avançado muito nos últimos anos para problemas $\mathcal{N} \mathcal{P}$-difíceis é o projeto de algoritmos de aproximação. É na aplicação dessa abordagem ao PSG que estamos interessados.

\section{Algoritmos de aproximação}

Seja $(G, c, R)$ uma instância do Problema de Steiner em Grafos e $A$ um algoritmo que produz uma árvore de Steiner $T$ de $(G, R)$. Dizemos que $A$ é um algoritmo de aproximação para o PSG se $A$ consome tempo polinomial no tamanho da instância e $c(T) \leq \alpha \cdot \operatorname{smt}(G, c, R)$ para qualquer instância $(G, c, R)$ do PSG, onde $\alpha$ é um número maior do que 1. Neste caso, dizemos que $A$ é uma $\alpha$-aproximação para o PSG e o número $\alpha$ é uma razão de aproximação do algoritmo $A$ para o PSG. Em geral, $\alpha$ pode ser uma função dependente da instância do problema, entretanto, neste traballho, estamos interessados em razão de aproximação $O(1)$, ou seja, em razão de aproximação constante. Algumas vezes, para simplicar o texto, usamos o termo algoritmo como sinônimo para algoritmo de aproximação.

O primeiro algoritmo de aproximação para o PSG é atribuído a Moore (1968), de acordo com Gilbert e Pollack [19]. O algoritmo constrói uma árvore geradora mínima de $(G[R], \dot{c})$, onde $\dot{c}$ é a restrição de $c$ a $G[R]$. Este algoritmo, que denotamos por $M S T$, tem uma razão de aproximação de 2 (mais exatamente, de $2+\frac{1}{n}$, onde $n$ é o número de terminais do grafo). Entre este algoritmo e o algoritmo das árvores 3-restritas outros algoritmos foram projetados $[32,41,36]$, porém todos com razão de aproximação de 2 . Abaixo, mostramos uma prova da razão de aproximação do algoritmo MST baseada na demostração apresentada por Vazirani [45].

Lema 1.5 Sejam $G$ um grafo completo, c uma função de $E_{G}$ em $Q_{>0}$ satisfazendo a desigualdade triangular e $R$ um conjunto de vértices de $G$. Se ć é a restrição de c a $G[R]$, então

$$
\operatorname{mst}(G[R], \dot{c}) \leq 2 \operatorname{smt}(G, c, R)
$$

Prova. Seja $T$ uma árvore de Steiner mínima de $(G, c, R)$. Vamos construir, a partir de $T$, uma árvore geradora de $G[R]$ de custo no máximo $2 c(T)$.

Para isso, duplique as arestas de $T$, obtendo um grafo euleriano $L$. Seja $P$ uma trilha euleriana em $L$; o custo desta trilha é $2 \operatorname{smt}(G, c, R)$. Sem perda de generalidade, podemos assumir que $P:=\left(v_{0}, e_{1}, \ldots, e_{l}, v_{l}\right)$, onde $v_{0}=v_{l}$ é terminal e folha em $T$.

Utilizando $P$ obtemos um circuito hamiltoniano $C:=\left(c_{0}, e_{1}^{\prime}, c_{1}, \ldots, c_{k-1}, e_{k}^{\prime}, c_{k}\right)$ em $G[R]$. O circuito $C$ foi obtido de maneira que $c_{0}=c_{k}=v_{0}$ e para cada par de vértices $c_{i-1}$ e $c_{i}, 1 \leq i \leq k$, existem dois terminais $v_{x}$ e $v_{y}$ em $P$ tais que $0<x<y \leq l$. Além disso, se existe um terminal $v_{t}$ em $P$ entre $v_{x}$ e $v_{y}$, então $v_{t}=c_{j}$ para algum $0 \leq j<i-1$. Note que $C$ pode ser obtido em tempo polinomial. 
Por causa da desigualdade triangular, o custo do circuito hamiltoniano $C$ não é maior do que o custo da trilha euleriana $P$. Jogando fora uma aresta deste circuito, obtemos uma árvore geradora $M$ de $G[R]$ que tem custo no máximo $2 \operatorname{smt}(G, c, R)$. Portanto, $\operatorname{mst}(G[R], \dot{c}) \leq 2 \operatorname{smt}(G, c, R)$.

A análise da razão de aproximação do algoritmo $M S T$ é justa, ou seja, existe uma instância do PSG para o qual o custo da solução produzida pelo algoritmo MST dividido pelo custo da árvore de Steiner mínima é tão próximo de 2 quanto se queira.

Veja na figura 1.7 tal instância. O grafo é composto por $n$ terminais e um vértice de Steiner. As arestas que ligam o vértice de Steiner aos terminais têm custo 1, enquanto que as outras arestas têm custo 2 . O custo de uma árvore geradora mínima para essa instância do problema é $2(n-1)$ e o custo de uma árvore de Steiner ótima é $n$.

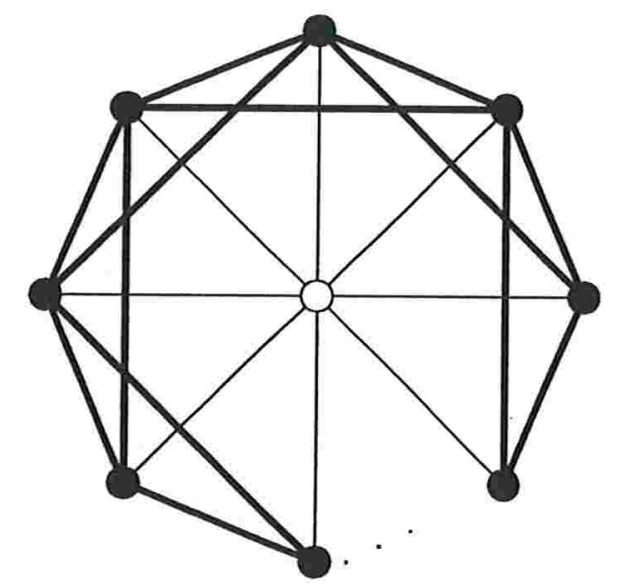

- Arestas de custo 2

- Arestas de custo 1

Figura 1.7: Grafo onde a razão de aproximação do algoritmo $M S T$ é $2+\frac{1}{n}$.

Lembrando que uma árvore geradora mínima de $(G[R], \dot{c})$ é uma árvore 2-restrita mínima de $(G, c, R)$, uma maneira de generalizar esse algoritmo, construindo boas aproximações para o PSG seria um algoritmo que devolvesse uma árvore $k$-restrita mínima para algum $k>2$ fixo (a figura 1.8 e o lema 1.6 abaixo comprovam a nossa afirmação). Borchers e Du [9] demonstraram uma fórmula para calcular o máximo da razão entre o custo de uma árvore $k$-restrita mínima e o custo de uma árvore de Steiner mínima para qualquer instância $(G, c, R)$ do PSG.

Lema 1.6 Seja $r_{k}$ o supremo da razão entre $\operatorname{smt}_{k}(G, c, R)$ e $\operatorname{smt}(G, c, R)$ para quais- 
quer $G$, c e $R$. Se $k=2^{l}+s$, ondel e s são naturais $e 0 \leq s<2^{l}$, então

$$
r_{k}=\frac{(l+1) 2^{l}+s}{l 2^{l}+s} .
$$

Para uma árvore 2-restrita mínima já vimos um algoritmo eficiente e, para encontrar uma árvore 3-restrita mínima existe uma $(1+\epsilon)$-aproximação probabilística polinomial [39], mas nenhuma demonstração de que o problema é $\mathcal{N} \mathcal{P}$-difícil. Em particular, pelo lema. 1.6, temos que $r_{2}=2$ e $r_{3}=\frac{5}{3}$.

Infelizmente, para $k \geq 4$, construir uma árvore $k$-restrita mínima é um problema $\mathcal{N} \mathcal{P}$ difícil [30]. Contudo, mesmo sendo difícil produzir tais árvores, elas serão utilizadas nos algoritmos e nas provas de razões de aproximações.

Com exceção do algoritmo de Berman e Ramayer os algoritmos que veremos são algoritmos gulosos (detalhes sobre algoritmos gulosos podem ser encontrados em [10]), ou seja, selecionam algumas árvores $k$-restritas, onde $k$ é uma constante fixa, cujos vértices de Steiner estarão presentes, junto com os terminais, na solução devolvida pelos algoritmos. Além disso, usaremos árvores $k$-restritas mínimas como limitantes superiores das soluções produzidas pelos algoritmos.

Vale ressaltar que o método guloso, apesar de ser o método utilizado pelo algoritmo com a menor razão de aproximação conhecida até o momento, não é o único para se construir soluções aproximadas para o PSG. Por exemplo, Williamson, Goemans, Mihail e Vazirani [46] projetaram um algoritmo para o PSG baseado no método primal-dual ${ }^{1}$.

\section{Inaproximabilidade}

A menor razão de aproximação conhecida para o PSG é 1,55. Uma pergunta natural é até que ponto podemos continuar procurando algoritmos que consigam melhorar essa razão de aproximação supondo que $\mathcal{P} \neq \mathcal{N} \mathcal{P}$. Nesta subseção mostraremos alguns resultados que impõe limites para uma razão de aproximação do PSG.

Arora, Lund, Motwani, Sudan e Szegedy [2] juntamente com uma redução, devida a Bern e Plassman [7], do problema da B-cobertura de vértices ${ }^{2}$ para o PSG mostram que existe uma constante $c>1$ tal que nenhum algoritmo de aproximação para o PSG tem razão de aproximação menor do que $c$, a menos que $\mathcal{P}=\mathcal{N} \mathcal{P}$. Denominamos uma tal constante $c$ de limite de aproximabilidade (approximation threshold).

Além disso, Bern e Plassman mostraram que, se existe um algoritmo com razão de aproximação $1+\epsilon$ para o PSG, então existe um algoritmo para o problema da $B$-cobertura de vértices com razão de aproximação $1+(1+B) \epsilon$. Portanto, bons algoritmos para o PSG implicam em bons algoritmos para o problema da $B$-cobertura de vértices. Com esse

\footnotetext{
${ }^{1}$ Detalhes sobre o método primal-dual podem ser encontrados em [16, 45].

${ }^{2} \mathrm{O}$ problema da $B$-cobertura de vértices consiste em descobrir um conjunto de vértices de custo mínimo tal que toda aresta do grafo tenha um dos extremos nesse conjunto onde o grau máximo dos vértices do grafo é menor ou igual a $B$.
} 
resultado, se conhecermos um limite de aproximabilidade para o problema da $B$-cobertura. de vértices, então temos um limite de aproximabilidade para o PSG.

Berman e Karpinski [5] mostraram que o melhor limite de aproximabilidade para o problema da 4-cobertura de vértices é de 1,0128. Logo temos um limite-de aproximabilidade de 1,0025 para o PSG. Recentemente Thimm [44], utilizando uma redução de Håstad [25] para o problema Max-E3-Lin-2, melhorou este resultado obtendo um valor de 1,0074 .

Podemos concluir que, mesmo com os avanços no projeto de algoritmos de aproximação, há uma grande diferença entre a razão de aproximação do melhor algoritmo conhecido até o presente e do melhor limite de aproximabilidade do PSG. 
Neste grafo completo apresentamos somente as arestas de custo 1. As demais arestas têm custo igual ao comprimento do menor caminho entre os dois vértices que elas ligam.

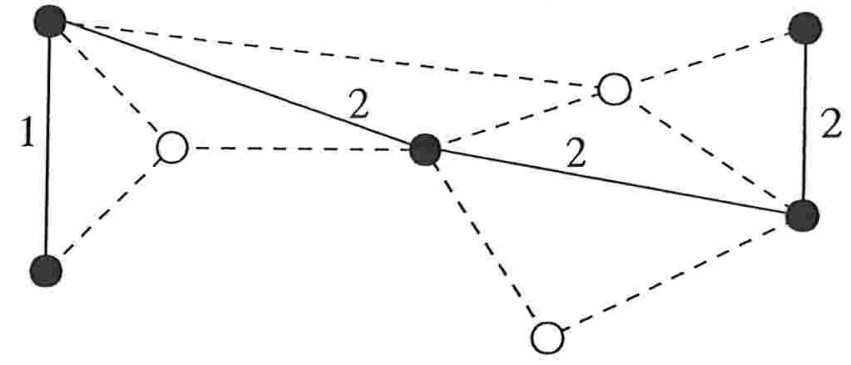

Árvore 2-restrita de custo mínimo Custo 7

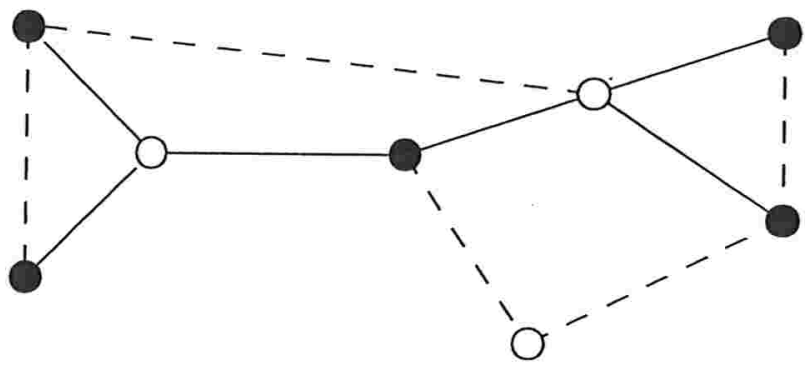

Árvore 3-restrita de custo mínimo Custo 6

Árvore 4-restrita de custo mínimo Árvore de Steiner ótima

Custo 5

$\bigcirc$ Vértices de Steiner

Arestas da árvore

Vértices Terminais

. . . . . Arestas de custo 1 não utilizadas

Figura 1.8: Exemplos de árvores k-restritas mínimas. 


\section{Capítulo 2}

\section{Algoritmo das árvores 3-restritas}

O algoritmo das árvores 3-restritas é um algoritmo guloso projetado por Zelikovsky [48] para o problema de Steiner em grafos. Este algoritmo foi o primeiro a superar o ingênuo algoritmo $M S T$, obtendo uma razão de aproximação de $\frac{11}{6}<1,834$. Zelikovsky utilizou árvores 3-restritas mínimas para inserir na solução produzida pelo seu algoritmo alguns vértices de Steiner. Esta abordagem inspirou vários outros algoritmos de aproximação para o PSG. A seguir, descrevemos o algoritmo das árvores 3-restritas.

\subsection{Descrição do algoritmo}

A entrada do algoritmo é um grafo completo $G$, uma função $c$ de $E_{G}$ em $Q_{>0}$ satisfazendo a desigualdade triangular e um conjunto $R$ de vértices - os terminais. Como saída, o algoritmo produz uma árvore de Steiner cujo custo é no máximo $\frac{11}{6}$ do custo de uma árvore de Steiner mínima de $(G, c, R)$.

Para um conjunto arbitrário $\tau$ de três terminais, que chamaremos de tripla, denote por $T_{\tau}$ uma árvore de Steiner mínima de $(G, c, \tau)$. Note que, neste caso, $T_{\tau}$ é também uma árvore 3-restrita mínima.

Vamos apresentar primeiramente uma variante do algoritmo de Zelikovsky. Depois comentaremos sobre o algoritmo original. O algoritmo começa com uma árvore geradora mínima $M$ de $(G[R], \dot{c})$, onde $\dot{c}$ é a restrição de $c$ a $G[R]$, e com um subgrafo $S$ de $G$. Inicialmente $S$ é a árvore $M$. Em cada iteração, o algoritmo escolhe uma tripla $\tau$ que maximiza $g(M, \tau)$. Se $g(M, \tau)$ é positivo, o algoritmo constrói uma árvore de Steiner mínima de $(G, c, \tau)$ e um conjunto remoção $D_{\tau}$ de $\tau$ em $M$. Então, o algoritmo diminui o custo $\dot{c}(e)$ de cada aresta $e$ em $G[R]$ que está na imagem da função projeção de $D_{\tau}$ em $\tau$. O custo de cada uma dessas arestas diminui de $g(M, \tau)$. Além disso, o algoritmo altera $M$ e $S$ adequadamente e pára quando não há mais triplas com ganho positivo. Neste ponto, o algoritmo devolve $S$. Veja a figura 2.1 .

Note que o algoritmo das árvores 3-restritas pode ser implementado de forma que seu consumo de tempo seja polinomial no tamanho da sua entrada. 
Algoritmo das árvores 3-restritas $(G, c, R)$

1 Seja $\dot{c}_{0}$ a restrição de $c$ a $G[R]$;

2 Seja $M_{0}$ uma árvore geradora mínima de $\left(G[R], \dot{c}_{0}\right)$;

$3 \quad S_{0}:=M_{0}$;

$4 \quad i:=1$;

5 Enquanto existir $\tau \subseteq R,|\tau|=3$, com $g\left(M_{i-1}, \tau\right)>0$ faça

$6 \quad$ Seja $\tau_{i} \subseteq R$ com $\left|\tau_{i}\right|=3$ e $g\left(M_{i-1}, \tau_{i}\right)$ máximo;

7 Seja $T_{i}$ uma árvore 3-restrita mínima de $\left(G, c, \tau_{i}\right)$;

8 Seja $D_{i}$ um conjunto remoção de $\tau_{i}$ em $M_{i-1}$;

9 Seja $p$ a função projeção de $D_{i}$ em $\tau_{i}$ relativa a $M_{i-1}$;

$10 \quad A_{i}:=\emptyset$;

$11 \quad c_{i}:=c_{i-1}$;

12 Para cada $e$ em $D_{i}$ faça

$13 \quad \dot{c}_{i}(p(e)):=\dot{c}_{i-1}(e)-g\left(M_{i-1}, \tau_{i}\right)$;

$14 \quad A_{i}:=A_{i} \cup\{p(e)\}$;

$15 \quad M_{i}:=M_{i-1}-D_{i}+A_{i}$;

$16 \quad S_{i}:=S_{i-1}-D_{i}+T_{i}$;

$17 \quad i:=i+1$;

$18 f:=i-1$;

19 Devolva $S_{f}$.

Figura 2.1: Algoritmo das árvores 3-restritas

\subsection{Análise do algoritmo das árvores 3-restritas}

No artigo original de Zelikovsky [48] havia um erro na análise do algoritmo. Esse erro foi explicitamente corrigido, apenas em 2001, no trabalho de Gröpl, Hougardy, Nierhoff e Prömel [21]. A nossa prova de uma razão de aproximação é baseada nesse trabalho.

A análise a seguir é longa e técnica. Os lemas 2.1 e 2.5 são utilizados na demonstração do teorema 2.6. Eles provam invariantes do algoritmo. O lema 2.2 é usado na demonstração do lema 2.3 e os lemas 2.3 e 2.4 são utilizado na demonstração do lema 2.5 .

Lema 2.1 Para todo $i=0, \ldots, f$, no algoritmo das árvores 3-restritas temos que $\dot{c}_{i}(e) \geq 0$ para toda aresta e de $G[R], M_{i}$ é uma árvore geradora mínima de $\left(G[R], \dot{c}_{i}\right)$ e $\dot{c}_{i}\left(M_{i}\right)=\dot{c}_{0}\left(M_{0}\right)-2 \sum_{j=1}^{i} g_{\tau_{j}}$, onde $g_{\tau_{j}}:=g\left(M_{j-1}, \tau_{j}\right)$.

Prova. A prova é por indução em $i$. Para $i=0$, o lema é trivial. Para $i>0$, suponha que $\dot{c}_{i-1}$ é não-negativo, que $M_{i-1}$ é uma árvore geradora mínima de $\left(G[R], \dot{c}_{i-1}\right)$ e que $\dot{c}_{i-1}\left(M_{i-1}\right)=\dot{c}_{0}\left(M_{0}\right)-2 \sum_{j=1}^{i-1} g_{\tau_{j}}$.

Vamos mostrar que $\dot{c}_{i}(e) \geq 0$ para toda aresta $e$ de $G[R]$. Note que as alterações de $\dot{c}_{i-1}$ para $\dot{c}_{i}$ ocorrem na linha 13 do algoritmo. Portanto basta mostrar que o novo custo atribuído é não-negativo. 
Digamos que $\tau_{i}=\{x, y, z\}, D_{i}=\{e, f\}$ e $p$ é a função projeção de $\tau_{i}$ em $D_{i}$ relativa a $M_{i-1}$. Sem perda de generalidade, suponha que $x$ é tal que $e$ é a aresta de custo máximo no caminho de $x$ a $y$ em $M_{i-1}$ e $f$ é a aresta de custo máximo no caminho de $x$ a $z$ em $M_{i-1}$. Se $v$ é o vértice de Steiner de $T_{i}$ (veja a figura 2.2 ), então

$$
c(x v)+c(v y) \geq c(x y) \geq \dot{c}_{i-1}(x y) \geq \dot{c}_{i-1}(e),
$$

onde a primeira desigualdade vale pela desigualdade triangular, a segunda pelo fato que $\dot{c}_{i-1}(e) \geq \dot{c}_{i}(e)^{1}$ para toda aresta $e$ de $G[R]$ e a terceira porque $M_{i-1}$ é uma árvore geradora mínima de $\left(G[R], \dot{c}_{i-1}\right)$. Utilizando a desigualdade acima podemos concluir que

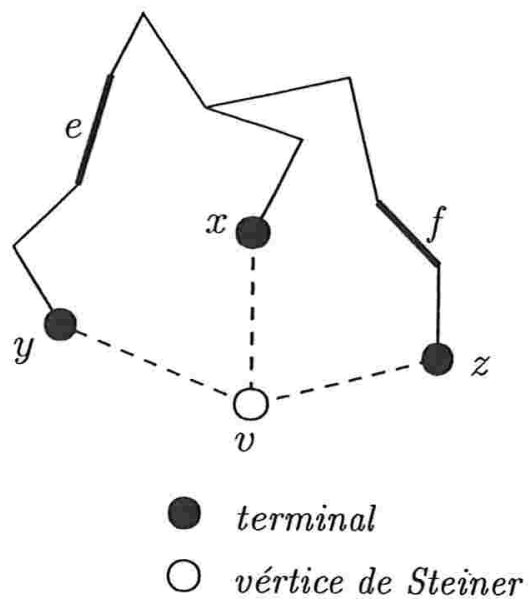

Figura 2.2: Linhas em negrito representam as arestas e e $f$, linhas normais são arestas de $M_{i-1}$ e linhas pontilhadas são as arestas de uma árvore de 3-retrita mínima de $(G, c, \tau)$.

$$
\begin{aligned}
\dot{c}_{i-1}(f)-g\left(M_{i-1}, \tau_{i}\right) & =\dot{c}_{i-1}(f)-\left(\dot{c}_{i-1}(e)+\dot{c}_{i-1}(f)-\operatorname{smt}\left(G, c, \tau_{i}\right)\right) \\
& =\operatorname{smt}\left(G, c, \tau_{i}\right)-\dot{c}_{i-1}(e) \\
& =c(x v)+c(v y)+c(v z)-\dot{c}_{i-1}(e)>0 .
\end{aligned}
$$

De forma análoga, podemos concluir que $\dot{c}_{i-1}(f)-g\left(M_{i-1}, \tau_{i}\right)>0$.

Pela definição de $D_{i}$ e $p, M_{i}$ é uma árvore. Além disso, como o custo $\dot{c}_{i}(p(e))<\dot{c}_{i-1}(e)$ para cada $e$ em $D_{i}$, temos que $p(e)$ é a aresta de custo mínimo de $\left(G[R], \dot{c}_{i}\right)$ no corte definido por uma das componentes de $M_{i-1}-e$. Portanto $M_{i}$ é uma árvore geradora mínima de $\left(G[R], \dot{c}_{i}\right)$. Quanto ao custo de $M_{i}$, temos que $\dot{c}_{i}\left(M_{i}\right)=\dot{c}_{i-1}\left(M_{i-1}\right)-2 g_{\tau_{i}}$. Assim, por indução,

$$
\dot{c}_{i}\left(M_{i}\right)=\dot{c}_{0}\left(M_{0}\right)-2 \sum_{j=1}^{i} g_{\tau_{j}}
$$

${ }^{1} \mathrm{Na}$ linha 13 do algoritmo note que $p(e)=x y$ não está em $M_{i-1}$ e $e$ é uma aresta de custo máximo no caminho de $x$ a $y$ em $M_{i-1}$, portanto $\dot{c}_{i-1}(p(e)) \geq \dot{c}_{i-1}(e)>\dot{c}_{i}(p(e))$ pois $g\left(M_{i-1}, \tau_{i}\right)>0$. Para as outras arestas temos que $\dot{c}_{i-1}(e) \geq \dot{c}_{i}(e)$. 
O próximo lema será usado na demonstração do lema 2.3.

Lema 2.2 No algoritmo das árvores 3-restritas, se $i \geq 1$, $\tau$ é uma tripla, $D$ é um separador de $\tau$ em $M_{i}$ e $\dot{c}_{i}(D)-\operatorname{smt}(G, c, \tau)>0$, então $D \cap A_{i}=\emptyset$.

Prova. Digamos que $A_{i}:=\left\{e^{\prime}, f^{\prime}\right\}$ e $D_{i}:=\{e, f\}$, onde $e^{\prime}:=p(e)$ e $f^{\prime}:=p(f)$. Suponha por contradição que $D \cap A_{i} \neq \emptyset$. Sem perda de generalidade, podemos assumir que $e^{\prime} \in D$. Se $f^{\prime} \in D$ também, então temos que

$$
\begin{aligned}
\dot{c}_{i}(D)-\operatorname{smt}(G, c, \tau) & =\dot{c}_{i}\left(e^{\prime}\right)+\dot{c}_{i}\left(f^{\prime}\right)-\operatorname{smt}(G, c, \tau) \\
& =\dot{c}_{i-1}(e)+\dot{c}_{i-1}(f)-\operatorname{smt}(G, c, \tau)-2 g\left(M_{i-1}, \tau_{i}\right) \\
& \leq g\left(M_{i-1}, \tau\right)-2 g\left(M_{i-1}, \tau_{i}\right) \\
& <0,
\end{aligned}
$$

pois e e $f$ são separadores de $\tau$ em $M_{i-1}, g\left(M_{i-1}, \tau_{i}\right) \geq g\left(M_{i-1}, \tau\right)$ e $g\left(M_{i-1}, \tau_{i}\right)>0$.

Por outro lado, se $f^{\prime} \notin D$, seja $h$ o elemento de $D$ distinto de $e^{\prime}$. Temos que

$$
\begin{aligned}
\dot{c}_{i}(D)-\operatorname{smt}(G, c, \tau) & =\dot{c}_{i}\left(e^{\prime}\right)+\dot{c}_{i}(h)-\operatorname{smt}(G, c, \tau) \\
& =\dot{c}_{i-1}(e)+\dot{c}_{i-1}(h)-\operatorname{smt}(G, c, \tau)-g\left(M_{i-1}, \tau_{i}\right) .
\end{aligned}
$$

Se $\{e, h\}$ é um separador de $\tau$ em $M_{i-1}$, então

$$
\dot{c}_{i}(D)-\operatorname{smt}(G, c, \tau) \leq g\left(M_{i-1}, \tau\right)-g\left(M_{i-1}, \tau_{i}\right) \leq 0 .
$$

Já se $\{e, h\}$ não é um separador de $\tau$ em $M_{i-1}$, então seja $C$ o único circuito em $M_{i}+f$. Considere o par de vértices $x$ e $y$ de $\tau$ tal que $h$ é a única aresta de $D$ no caminho $P$ de $x$ a $y$ em $M_{i}$. Se $\{e, h\}$ não é um separador então $P$ contém $f$; do contrário $P$ seria o caminho de $x$ a $y$ em $M_{i-1}$. O caminho $P^{\prime}$ de $x$ a $y$ em $M_{i-1}$ contém uma aresta de $C$ se e só se $P$ não a contém. Em particular, se $P^{\prime}$ não contém $h$ então $h$ está em $C$. Neste caso, $\{e, f\}$ é um separador de $\tau$ em $M_{i-1}$ e $\dot{c}_{i-1}(f) \geq \dot{c}_{i-1}(h)$. Veja a figura 2.3. Assim, temos que

$$
\begin{aligned}
\dot{c}_{i}(D)-\operatorname{smt}(G, c, \tau) & \leq \dot{c}_{i-1}(e)+\dot{c}_{i-1}(f)-\operatorname{smt}(G, c, \tau)-g\left(M_{i-1}, \tau_{i}\right) \\
& \leq g\left(M_{i-1}, \tau\right)-g\left(M_{i-1}, \tau_{i}\right) \\
& \leq 0
\end{aligned}
$$

Nos vários casos, chegamos a uma contradição da hipótese que $\dot{c}_{i}(D)-\operatorname{smt}(G, c, \tau)>0$. Portanto podemos concluir que $D \cap A_{i}=\emptyset$.

O lema 2.3 abaixo, mais técnico, é uma peça central na demonstração de que, em cada. iteração do algoritmo, todas as arestas do conjunto $D_{i}$ estão em $S_{i-1}$. Isso será usado na. prova de que $S_{f}$ contém uma árvore de Steiner de $(G, R)$. 


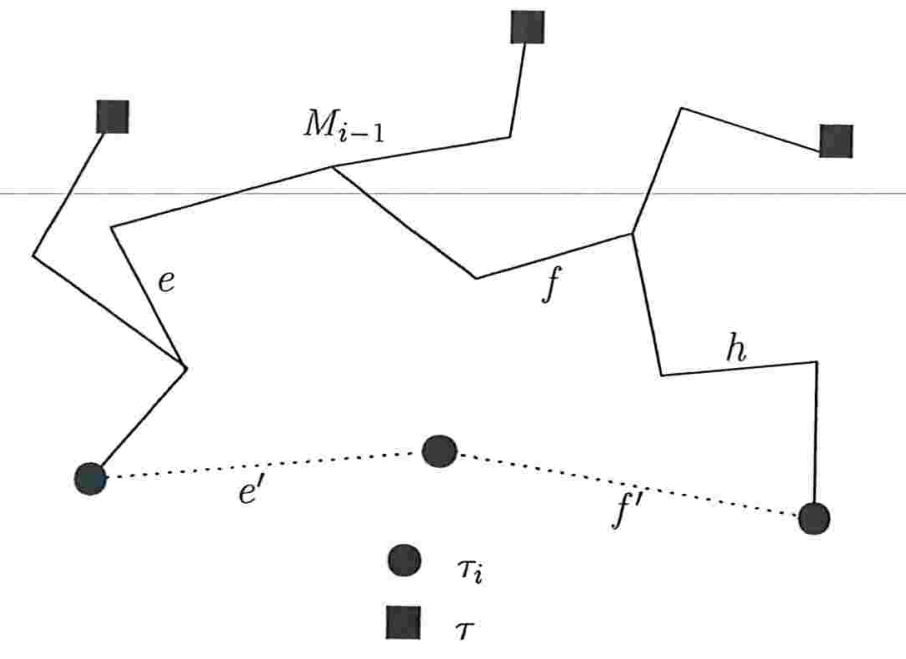

Figura 2.3: Temos que $M_{i}:=M_{i-1}-\{e, f\}+\left\{e^{\prime}, f^{\prime}\right\},\left\{e^{\prime}, h\right\}$ é um separador de $\tau$ em $M_{i}$, $\{e, h\}$ não é um separador de $\tau$ em $M_{i-1}$ mas $\{e, f\}$ é.

Lema 2.3 Sejam $i \geq 1$ e a em $M_{i}$. Se $\tau$ é uma tripla tal que $D:=\{a, b\}$ é um separador de $\tau$ em $M_{i}$ para algum $b$ e $\dot{c}_{i}(D)-\operatorname{smt}(G, c, \tau)>0$, então $a \in M_{i-1}$ e existe uma tripla $\tau^{\prime}$ e uma aresta $b^{\prime}$ em $M_{i-1}$ tal que $D^{\prime}:=\left\{a, b^{\prime}\right\}$ é um separador de $\tau^{\prime}$ em $M_{i-1} e$ $\dot{c}_{i-1}\left(D^{\prime}\right)-\operatorname{smt}\left(G, c, \tau^{\prime}\right) \geq \dot{c}_{i}(D)-\operatorname{smt}(G, c, \tau)$.

Prova. Sejam $D_{i}:=\{e, f\}$ um separador de $\tau_{i}$ em $M_{i-1}$ e $A_{i}:=\left\{e^{\prime}, f^{\prime}\right\}$ a imagem da função projeção de $D_{i}$ de $\tau_{i}$ relativa a $M_{i-1}$. Pelo lema $2.2, D \cap A_{i}=\emptyset$, ou seja, $\{a, b\} \subseteq M_{i-1}$. Sejam $A, B$ e $C$ os componentes de $M_{i}-\left\{e^{\prime}, f^{\prime}\right\}$ como na figura 2.4. Sejam $C_{1}$ o único circuito em $M_{i}+e$ e $C_{2}$ o único circuito em $M_{i}+f$.

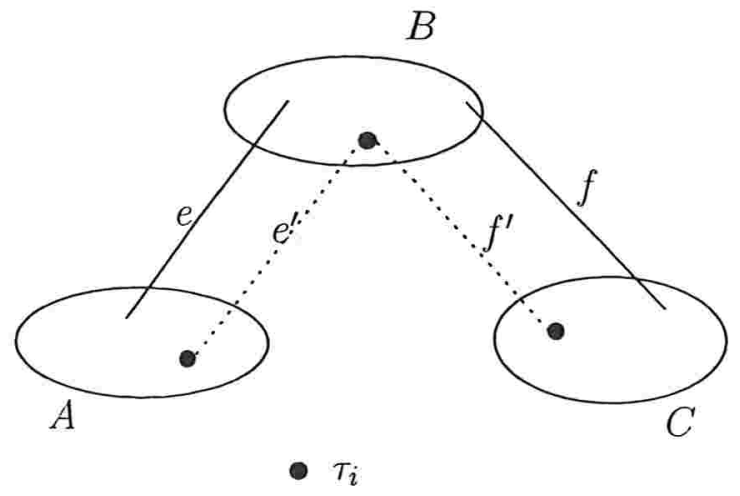

Figura 2.4: 
Caso 1: $a \notin C_{1} \cup C_{2}$

Sem perda de generalidade, suponha que $a \in E_{A}$ (de forma análoga vale para $E_{B}$ e $E_{C}$ ). Sejam $A^{\prime}$ e $A^{\prime \prime}$ os componentes de $A-a$ e suponha que $A^{\prime \prime}$ não contém nenhum extremo de $e^{\prime}$ e $f^{\prime}$. Note que $A^{\prime \prime}$ também não contém os extremos de $e$ ou $f$ e contém pelo menos um e no máximo dois elementos de $\tau$ pois $\{a, b\}$ é um separador de $\tau$ em $M_{i}$. Veja a figura 2.5. Se $A^{\prime \prime}$ contém dois elementos, então $\{a, b\}$

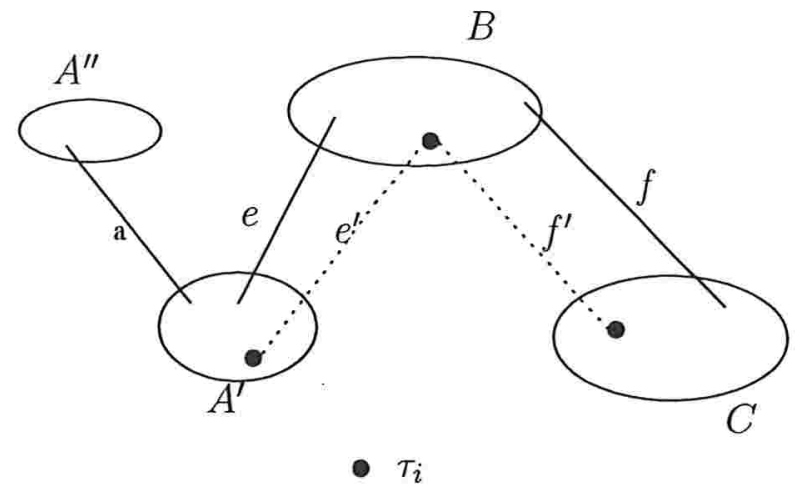

Figura 2.5:

é um separador de $\tau$ em $M_{i-1}$. Assim, tome $\tau^{\prime}:=\tau$ e $b^{\prime}:=b$ e vale o lema.

Se $A^{\prime \prime}$ contém exatamente um elemento de $\tau$ e os outros dois elementos de $\tau$ estão em $A^{\prime}$ ou $B$ ou $C$, então $\{a, b\}$ é um separador de $\tau$ em $M_{i-1}$ e o lema é verdadeiro. Senão, apenas um entre $A^{\prime}, B$ e $C$ não contém elementos de $\tau$. Neste caso, se $\{a, b\}$ não é um separador de $\tau$ em $M_{i-1}$, então ou $e$ ou $f$ ou ambos estão no caminho entre esses vértices. Tome $\tau^{\prime}:=\tau$ e $b^{\prime}$ de custo máximo entre os elementos de $\{e, f\}$ que estão no caminho entre esses vértices. Note que $\dot{c}\left(b^{\prime}\right) \geq \dot{c}(b)$, pois $b$ está em algum dos circuitos $C_{1}$ e $C_{2}$. Assim, temos que

$$
\begin{aligned}
\dot{c}_{i-1}\left(D^{\prime}\right)-\operatorname{smt}\left(G, c, \tau^{\prime}\right) & =\dot{c}_{i-1}(a)+\dot{c}_{i-1}\left(b^{\prime}\right)-\operatorname{smt}(G, c, \tau) \\
& \geq \dot{c}_{i-1}(a)+\dot{c}_{i-1}(b)-\operatorname{smt}(G, c, \tau) \\
& =\dot{c}_{i}+\dot{c}_{i}(b)-\operatorname{smt}(G, c, \tau) \\
& =\dot{c}_{i}(D)-\operatorname{smt}(G, c, \tau) .
\end{aligned}
$$

Caso 2: $a \in C_{1} \cup C_{2}$

Caso 2.1: $a \in E_{A}\left(a \in E_{C}\right.$ é análogo)

Sejam $A^{\prime}$ e $A^{\prime \prime}$ os componentes de $A-a$ onde $A^{\prime}$ contém um extremo de $e^{\prime}$. Note que $A^{\prime \prime}$ contém pelo menos um e no máximo dois elementos de $\tau$ pois $\{a, b\}$ é um separador de $\tau$ em $M_{i}$. Veja a figura 2.6. Se $A^{\prime \prime}$ tem dois elementos 


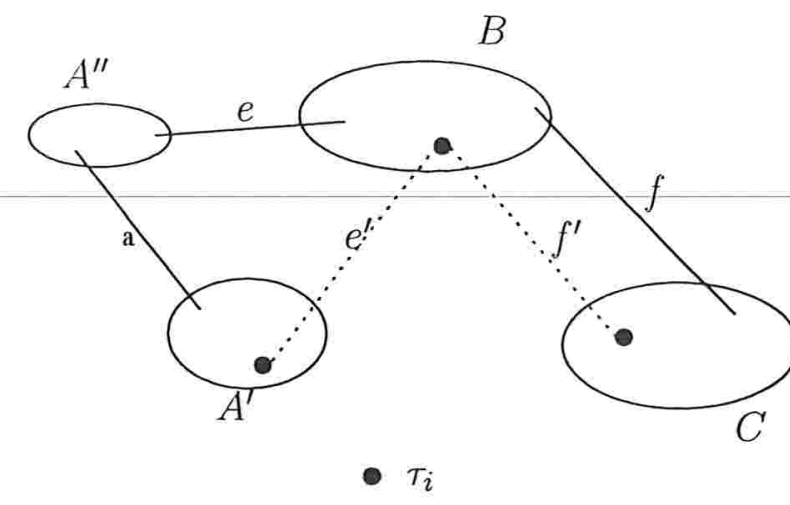

Figura 2.6:

de $\tau$ e $A^{\prime}$ tem um ou $A^{\prime}$ tem dois elementos de $\tau$, então $\{a, b\}$ é um separador de $\tau$ em $M_{i-1}$. Caso contrário há pelo menos um elemento de $\tau$ em $B \cup C$. Se $A^{\prime}$ contém um elemento de $\tau$, então, supondo que $b$ não separa $\tau$ em $M_{i-1}$, ou $e$ ou $f$ ou ambos separam em $M_{i-1}$ um par de elementos de $\tau$. Tome $\tau^{\prime}:=\tau$ e $b^{\prime}$ em $\{e, f\}$ que separa $\tau$ e tem custo máximo. De modo análogo ao caso 1 , temos que $\dot{c}\left(b^{\prime}\right) \geq \dot{c}(b)$ e o lema vale. Se $A^{\prime}$ não contém nenhum elemento de $\tau$, então $a$ não separa nenhum elemento de $\tau$ em $M_{i-1}$ e $\{e, b\}$ é um separador de $\tau$ em $M_{i-1}$. Assim, tome $\tau^{\prime}:=\tau_{i}$ e $b^{\prime}:=f$,

$$
\begin{aligned}
\dot{c}_{i-1}\left(D^{\prime}\right)-\operatorname{smt}\left(G, c, \tau^{\prime}\right) & =\dot{c}_{i-1}(a)+\dot{c}_{i-1}(f)-\operatorname{smt}\left(G, c, \tau_{i}\right)+\dot{c}_{i-1}(e)-\dot{c}_{i-1}(e) \\
& =\dot{c}_{i-1}\left(D_{i}\right)-\operatorname{smt}\left(G, c, \tau_{i}\right)+\dot{c}_{i}(a)-\dot{c}_{i-1}(e) \\
& =g\left(M_{i-1}, \tau_{i}\right)+\dot{c}_{i}(a)-\dot{c}_{i-1}(e) \\
& \geq g\left(M_{i-1}, \tau_{i}\right) \\
& \geq g\left(M_{i-1}, \tau\right) \\
& =\dot{c}_{i-1}(a)+\dot{c}_{i-1}(b)-\operatorname{smt}(G, c, \tau) \\
& =\dot{c}_{i}(a)+\dot{c}_{i}(b)-\operatorname{smt}(G, c, \tau) \\
& =\dot{c}_{i}(D)-\operatorname{smt}(G, c, \tau),
\end{aligned}
$$

onde a desigualdade (2.1) vale pois a está no caminho em $M_{i-1}$ entre o vértice de $\tau_{i}$ que está em $A^{\prime}$ e o vértice de $\tau_{i}$ que está em $B$ e $\dot{c}_{i-1}(a) \leq \dot{c}_{i-1}(e)$.

Caso 2.2: $a \in E_{B}$

Sejam $B^{\prime}$ e $B^{\prime \prime}$ os componentes de $B-a$ onde $B^{\prime}$ contém o extremo comum de $e^{\prime}$ e $f^{\prime}$. Note que $B^{\prime \prime}$ contém pelo menos um e no máximo dois elementos de $\tau$. Veja a figura 2.7. Se $B^{\prime \prime}$ tem dois elementos de $\tau$ e $B^{\prime}$ tem um elemento de $\tau$, então $\{a, b\}$ é um separador de $\tau$ em $M_{i-1}$. Do mesmo modo, se $B^{\prime \prime}$ tem um elemento de $\tau$ e $B^{\prime}$ ou $A$ ou $C$ tem dois elementos de $\tau$, então $\{a, b\}$ é um separador de $\tau$ em $M_{i-1}$. 


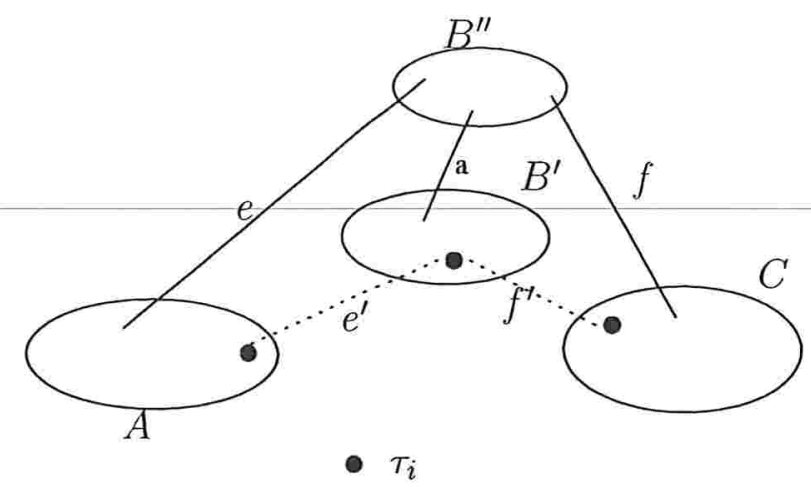

Figura 2.7:

Senão há pelo menos um elemento de $\tau$ em $A \cup C$. Se $A \cup C$ contém exatamente um elemento de $\tau$, então tome $\tau^{\prime}:=\tau$ e $b^{\prime}:=e$ ou $f$ dependendo desse elemento estar em $A$ ou $C$. Note que neste caso $b$ está em $C_{1}$ ou $C_{2}$, assim $\dot{c}_{i-1}\left(b^{\prime}\right) \geq$ $\dot{c}_{i-1}(b)$ e vale o lema (análoga à uma situação do caso 1 ).

Se $A \cup C$ contém dois elementos de $\tau$, então $a$ não separa nenhum elemento de $\tau$ em $M_{i-1}, \operatorname{logo}$ tome $\tau^{\prime}:=\tau_{i}$ e $b^{\prime}$ em $\{e, f\}$ de custo máximo em $M_{i-1}$. Como no caso 1 , o lema vale pois, como no caso 2.1 , temos que $\dot{c}_{i-1}\left(D^{\prime}\right)-\operatorname{smt}\left(G, c, \tau^{\prime}\right) \geq$ $\dot{c}_{i}(D)-\operatorname{smt}(G, c, \tau)$

Pelos casos 1 e 2 podemos concluir que o lema é verdadeiro.

Finalmente, o lema abaixo garante que as arestas dos conjuntos remoção escolhidos pelo algoritmo não tiveram o seu custo alterado durante a execução do algoritmo, ou seja, não fizeram parte de nenhum conjunto $A_{i}$ de uma iteração anterior.

Lema 2.4 Se $i \geq 0$, $\tau$ é uma tripla, $D$ é um conjunto separador de $\tau$ em $M_{i}$ e $\dot{c}_{i}(D)-$ $\operatorname{smt}(G, c, \tau)>0$, então $D \cap \bigcup_{j=1}^{i} A_{j}=\emptyset$.

Prova. A prova é por indução em $i$. Para $i=0$ o lema é trivial. Por indução, suponha que o lema vale para $i-1$. Agora provaremos que o lema vale para $i$.

Primeiramente, pelo lema 2.2, $D \cap A_{i}=\emptyset$. Agora, seja $a$ um elemento arbitrário de $D$. Vamos mostrar que $a \notin \bigcup_{j=1}^{i-1} A_{j}$. Como $i \geq 1, D$ é um separador de $\tau$ em $M_{i}, a \in D$ e $\dot{c}_{i}(D)-\operatorname{smt}(G, c, \tau)>0$, pelo lema 2.3, $a \in M_{i-1}$ e existe $b$ em $M_{i-1}$ e uma tripla $\tau^{\prime}$ tal que $D^{\prime}:=\left\{a, b^{\prime}\right\}$ é um separador de $\tau^{\prime}$ em $M_{i-1} \operatorname{com} \dot{c}_{i-1}\left(D^{\prime}\right)-\operatorname{smt}\left(G, c, \tau^{\prime}\right)>0$. Mas então, pela hipótese de indução, $D^{\prime} \cap \bigcup_{j=1}^{i-1} A_{j}=\emptyset$, ou seja, $a \notin \bigcup_{j=1}^{i-1} A_{j}$. Como isso vale para um elemento arbitrário de $D$, concluímos que $D \cap \bigcup_{j=1}^{i-1} A_{j}=\emptyset$ e portanto $D \cap \bigcup_{j=1}^{i} A_{j}=\emptyset$. 
A seguir mostraremos que $S_{f}$ contém uma árvore de Steiner de $(G, R)$ e que seu custo não passa de $c\left(S_{0}\right)-\sum_{j=1}^{f} g\left(M_{j-1}, \tau_{j}\right)$.

Para tanto, considere uma variante do algoritmo em que, para cada árvore $T_{i}$ escolhida pelo algoritmo na linha 7 , é feita uma cópia do vértice de Steiner de $T_{i}$. Com essas cópias, podemos assumir que $T_{i}$ utiliza um vértice de Steiner nunca antes utilizado (eventualmente tal vértice será uma cópia de um vértice utilizado anteriormente). Para essa variante do algoritmo, podemos mostrar o seguinte lema.

Lema 2.5 Para todo $i, 0 \leq i \leq f$, no algoritmo das árvores 3-restritas temos que $S_{i}$ é uma árvore de Steiner de $(G, R), c\left(S_{i}\right)=c\left(S_{0}\right)-\sum_{j=1}^{i} g\left(M_{j-1}, \tau_{j}\right)$ e cada aresta de $M_{i} \cap S_{i}$ separa os mesmos pares de terminais em $M_{i}$ e $S_{i}$.

Prova. A prova é por indução em $i$. Para $i=0$ o lema é trivial. Para $i>0$, suponha que $S_{i-1}$ é uma árvore de Steiner de $(G, R)$, que $c\left(S_{i-1}\right)=c\left(S_{0}\right)-\sum_{j=1}^{i-1} g\left(M_{j-1}, \tau_{j}\right)$ e que cada aresta de $M_{i-1} \cap S_{i-1}$ separa os mesmos pares de terminais em $M_{i-1}$ e $S_{i-1}$.

Pelo lema 2.4, $D_{i} \cap \bigcup_{j=1}^{i-1} A_{j}=\emptyset$. Isso implica que $D_{i} \subseteq\left(M_{i-1} \cap S_{i-1}\right)$. Assim, em $M_{i-1}-D_{i}$, dois terminais estão separados se e só se estão separados em $S_{i-1}-D_{i}$. Como $A_{i}$ é uma árvore geradora de $G\left[\tau_{i}\right]$ e $T_{i}$ é uma árvore de Steiner de $\left(G, \tau_{i}\right)$ que não usa vértices de Steiner de $S_{i-1}$, temos que $S_{i}$ é uma árvore de Steiner de $(G, R)$ e cada aresta de $M_{i} \cap S_{i}$ separa os mesmos pares de terminais em $M_{i}$ e $S_{i}$.

Quanto ao custo de $S_{i}$, temos que $c\left(S_{i}\right)=c\left(S_{i-1}\right)-c\left(D_{i}\right)+c\left(T_{i}\right)=c\left(S_{i-1}\right)-g\left(M_{i-1}, \tau_{i}\right)$ e, por indução, $c\left(S_{i}\right)=c\left(S_{0}\right)-\sum_{j=1}^{i} g\left(M_{j-1}, \tau_{j}\right)$.

Contraindo-se os vértices de Steiner de $S_{f}$ que são cópias de um mesmo vértice, obtém-se o $S_{f}$ devolvido pelo algoritmo das árvores 3-restritas. Tal operação de contração mantém $S_{f}$ conexo e não altera o custo já que não há custo nas arestas entre os vértices de Steiner duplicados. Podemos concluir então que o $S_{f}$ devolvido pelo algoritmo contém uma árvore de Steiner de $(G, R)$ e que $c\left(S_{f}\right)=c\left(S_{0}\right)-\sum_{j=1}^{f} g\left(M_{j-1}, \tau_{j}\right)$.

Finalmente temos o teorema mostrando uma razão de aproximação para o algoritmo das árvores 3-restritas.

Teorema 2.6 Sejam $G$ um grafo completo, c uma função de $E_{G}$ em $Q_{>0}$ satisfazendo a desigualdade triangular e $R$ um conjunto de vértices de $G$. $O$ custo da solução do algoritmo das árvores 3-restritas para $(G, c, R)$ é no máximo $\frac{11}{6}$ do custo de uma árvore de Steiner mínima de $(G, c, R)$.

Prova. Denote $g\left(M_{i-1}, \tau_{i}\right)$ por $g_{\tau_{i}}$. Como $\dot{c}_{0}\left(M_{0}\right)=c\left(S_{0}\right)$, pelos lemas 2.1 e 2.4, temos que,

$$
c\left(S_{f}\right) \leq c\left(S_{0}\right)-\sum_{i=0}^{f-1} g_{\tau_{i}}=c\left(S_{0}\right)-\left(\frac{\dot{c}_{f}\left(M_{f}\right)-\dot{c}_{0}\left(M_{0}\right)}{2}\right) \leq \frac{\dot{c}_{0}\left(M_{0}\right)+\dot{c}_{f}\left(M_{f}\right)}{2} .
$$


Agora mostraremos que $\dot{c}_{f}\left(M_{f}\right) \leq \operatorname{smt}_{3}(G, c, R)$. Seja $T^{*}$ uma árvore 3-restrita mínima de $(G, c, R)$ e $\tau_{1}^{*}, \ldots, \tau_{l}^{*}$ o conjunto de terminais de cada um dos componentes cheios de $T^{*}$ (note que $2 \leq\left|\tau_{i}^{*}\right| \leq 3$ para $1 \leq i \leq l$ ). Como $\operatorname{mst}\left(G[R], \dot{c}\left[\tau_{1}^{*}, \ldots, \tau_{l}^{*}\right]\right.$ ) $=0$, podemos escrever o custo de $M_{f}$ como

$$
\begin{aligned}
\dot{c}_{f}\left(M_{f}\right) & =m s t\left(G[R], \dot{c}_{f}\right)-\operatorname{mst}\left(G[R], \dot{c}_{f}\left[\tau_{0}^{*}, \ldots, \tau_{l}^{*}\right]\right) \\
& =\sum_{k=1}^{l}\left(\operatorname{mst}\left(G[R], \dot{c}_{f}\left[\tau_{1}^{*}, \ldots, \tau_{k-1}^{*}\right]\right)-\operatorname{mst}\left(G[R], \dot{c}_{f}\left[\tau_{1}^{*}, \ldots, \tau_{k}^{*}\right]\right)\right) \\
& \leq \sum_{k=1}^{l}\left(\operatorname{mst}\left(G[R], \dot{c}_{f}\right)-\operatorname{mst}\left(G[R], \dot{c}_{f}\left[\tau_{k}^{*}\right]\right)\right) \\
& =\sum_{k=1}^{l}\left(g\left(M_{f}, \tau_{k}^{*}\right)+\operatorname{smt}\left(G, c, \tau_{k}^{*}\right)\right) \\
& \leq \sum_{k=1}^{l} \operatorname{smt}\left(G, c, \tau_{k}^{*}\right)=c\left(T_{3}^{*}\right) .
\end{aligned}
$$

A desigualdade (2.3) vale por (1.1) e pelo corolário 1.3 e a desigualdade (2.4) vale pela linha 5 do algoritmo que garante que $g\left(M_{f}, \tau_{k}^{*}\right) \leq 0$ para todo $\tau_{k}^{*}$.

Como $\dot{c}_{0}\left(M_{0}\right)=\operatorname{smt}_{2}(G, c, R)$ e $\dot{c}_{f}\left(M_{f}\right) \leq \operatorname{smt}_{3}(G, c, R)$, podemos substituir esses valores na desigualdade (2.2) e dividir pelo custo de uma árvore de Steiner mínima de $(G, c, R)$, ficando com

$$
\begin{aligned}
\frac{c\left(S_{f}\right)}{\operatorname{smt}(G, c, R)} & \leq \frac{s m t_{2}(G, c, R)}{2 \operatorname{smt}(G, c, R)}+\frac{s m t_{3}(G, c, R)}{2 s m t(G, c, R)} \\
& \leq \frac{r_{2}+r_{3}}{2} \\
& =\frac{2}{2}+\frac{5 / 3}{2} \\
& =\frac{11}{6} .
\end{aligned}
$$

Onde (2.5) vem de $r_{k}:=\max \left\{\frac{\operatorname{smt} t_{k}(G, c, R)}{\operatorname{smt}(G, c, R)}\right\}$ e a igualdade (2.6) vale pelo lema 1.6.

Infelizmente, não existe na literatura uma família de instâncias tal que a aplicação deste algoritmo resulte na razão de aproximação anunciada. Logo existe a possibilidade deste algoritmo ter uma razão de aproximação menor do que $\frac{11}{6}$.

\subsection{Algoritmo original de Zelikovsky}

O algoritmo originalmente proposto por Zelikovsky diferia em vários pontos da versão apresentada na figura 2.1. Primeiramente, na linha $13, c_{i}(p(e))$ era zerado. O algoritmo 
não construía iterativamente as árvores $S_{0}, \ldots, S_{f}$. Ele apenas guardava em um conjunto $W$ os vértices de Steiner das árvores $\tau_{1}, \ldots, \tau_{f}$ e no fim devolvia uma árvore geradora mínima de $(G[R \cup W], c)$. Veja a figura 2.8.

A análise apresentada para a variante pode ser facilmente alterada para o algoritmo original. Basta notar que o lema 2.4 continua válido mesmo com a alteração na linha 13 e que $\operatorname{mst}\left(G\left[R \cup W_{f}\right], c\right) \leq c\left(S_{f}\right)$

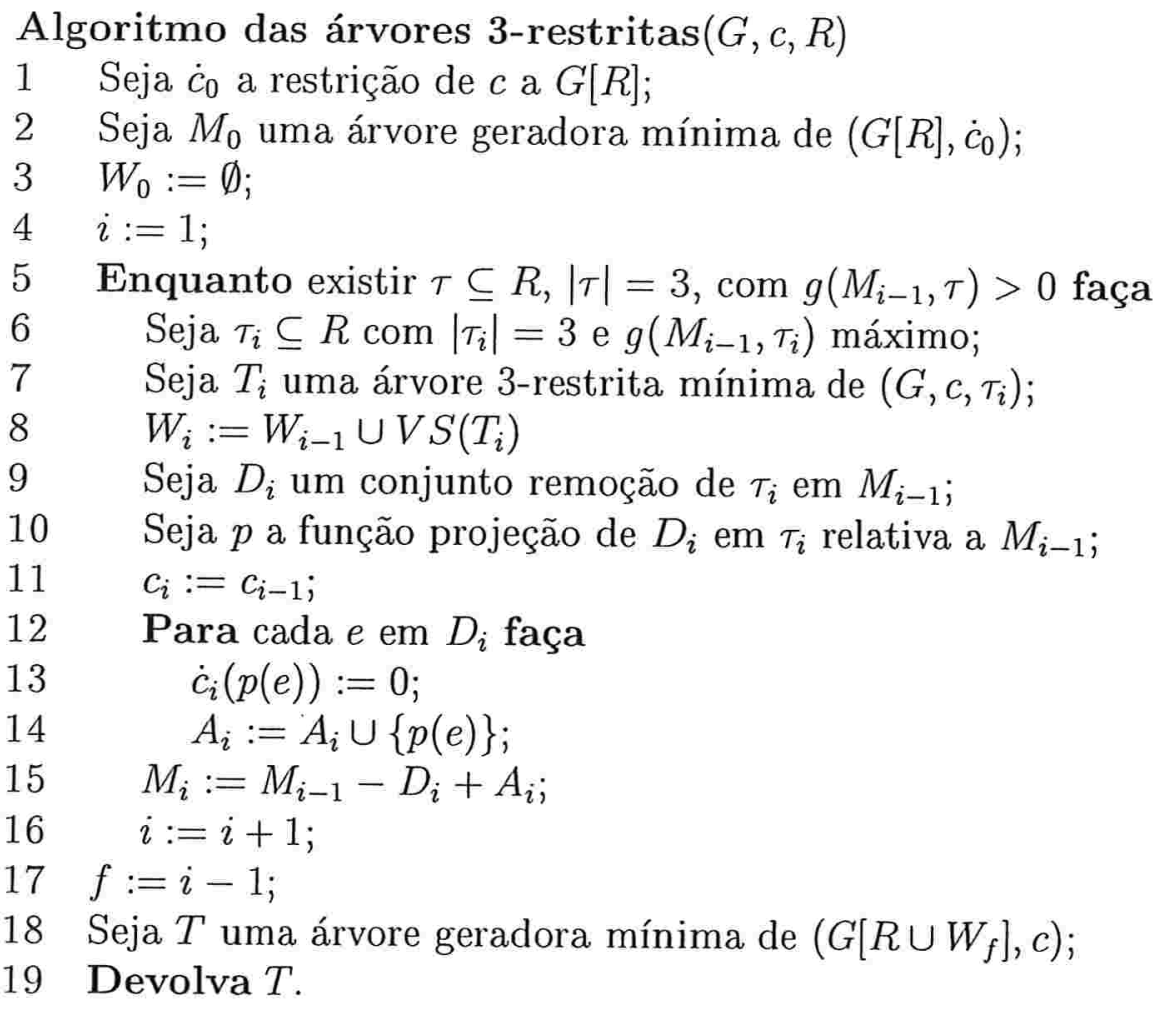

Figura 2.8: Algoritmo das árvores 3-restritas original 


\section{Capítulo 3}

\section{Algoritmo de Berman e Ramayer}

O algoritmo descrito no capítulo anterior utiliza árvores 3-restritas mínimas para escolher os vértices de Steiner que estarão na solução produzida pelo algoritmo. Uma idéia natural para melhorar esse algoritmo é a utilização de árvores de Steiner mínimas sobre conjuntos de até $k$ terminais, onde $k$ é uma constante maior ou igual a 3 .

Berman e Ramayer [6] apresentaram um algoritmo que utiliza esta idéia resultando em uma razão de aproximação menor que $\frac{11}{6}$ para o PSG.

\subsection{Descrição do algoritmo}

$\mathrm{Na}$ verdade, Berman e Ramayer descreveram uma família de algoritmos: um algoritmo para cada $k \geq 3$. A entrada de cada algoritmo é um grafo completo $G$, uma função $c$ de $E_{G}$ em $Q_{>0}$ satisfazendo a desigualdade triangular e um conjunto $R$ de vértices - os terminais. Como saída, o algoritmo produz uma árvore de Steiner de $(G, R)$ cujo custo dividido pelo custo de uma solução ótima do PSG para $(G, c, R)$ é no máximo

$$
r_{2}-\sum_{i=3}^{k} \frac{r_{i-1}-r_{i}}{i-1}
$$

Pelo lema 1.6, para $k$ suficientemente grande a razão de aproximação do algoritmo de Berman e Ramayer se aproxima de 1,734 .

Note que quando $k=3$ a expressão acima coincide com a razão demonstrada para o algoritmo das árvores 3-restritas. Embora neste caso a delimitação na razão de aproximação seja a mesma para os dois algoritmos, eles são diferentes, ou seja, para uma mesma instância, eles podem produzir soluções diferentes. Há no entanto uma forma de modificar o algoritmo de Berman e Ramayer de forma que, para $k=3$, ele funcione exatamente como o algoritmo das árvores 3-restritas.

O algoritmo de Berman e Ramayer, para um certo $k$, é dividido em duas fases: a fase de avaliação e a fase de construção, que passamos a descrever. 


\section{Fase de avaliação}

Seja $\dot{c}$ a restrição de $c$ a $G[R]$. Esta fase inicia com uma árvore geradora mínima $M$ de $(G[R], \dot{c})$ e pilhas $\pi_{j}(3 \leq j \leq k)$ inicialmente vazias. Esta fase processa, em ordem crescente de cardinalidade, todos conjuntos $\tau$ de terminais que têm cardinalidade entre 3 e $k$.

Em cada iteração, esta fase toma um conjunto $\tau$ de terminais e constrói uma árvore de Steiner mínima $T_{\tau}^{*}$ de $(G, c, \tau)$, um conjunto remoção $D$ de $\tau$ em $M$ e a função projeção $p$ de $D$ em $\tau$ relativa a $M$.

Se o ganho de $\tau$ em $M$ é positivo, empilha-se algumas informações para usar na fase de construção, altera-se convenientemente o custo $\dot{c}$ na imagem de $p$ e modifica-se a árvore $M$, removendo cada aresta $e$ de $D$ e adicionando $p(e)$. A fase termina quando todos os conjuntos são analisados e ela devolve uma árvore geradora mínima de $(G[R], \dot{c})$ e as pilhas $\pi_{j}$ para $3 \leq j \leq k$. Abaixo segue a descrição precisa da fase de avaliação.

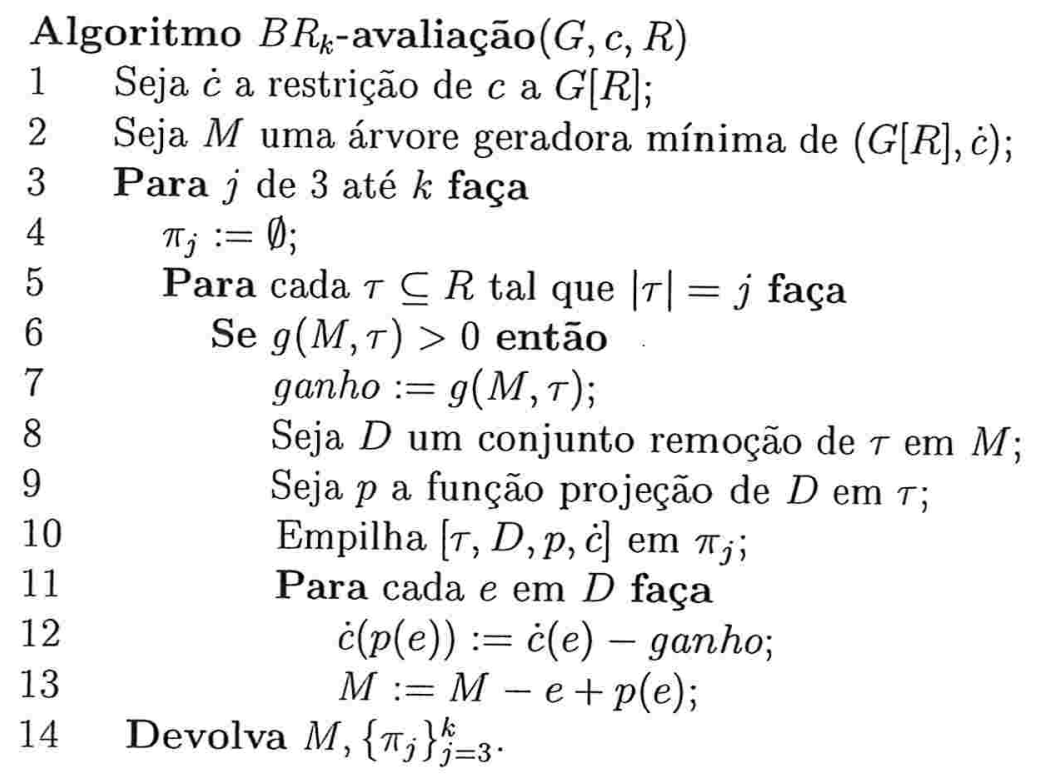

Figura 3.1: Fase de avaliação

Sejam $M_{2}$ e $\dot{c}_{2}$ os valores iniciais de $M$ e $\dot{c}$ e, para $3 \leq j \leq k$, sejam $M_{j}$ e $\dot{c}_{j}$ os valores de $M$ e $\dot{c}$ após o processamento de todos os conjuntos $\tau$ onde $|\tau|=j$, ou seja, ao final da iteração $j$ do para da linha 3. Denotando por $Q(j)$ o conjunto dos $\tau$ armazenados em $\pi_{j}$ e por $g_{\tau}$ o valor de $g(M, \tau)$ na linha 6 da iteração em que $\tau$ é processado, temos o seguinte invariante do algoritmo $B R_{k}$-avaliação.

Lema 3.1 Para todo $j, 2 \leq j \leq k, M_{j}$ é uma árvore geradora mínima de $\left(G[R], \dot{c}_{j}\right)$. 
Além disso,

$$
\dot{c}_{j}\left(M_{j}\right)= \begin{cases}\operatorname{mst}\left(G[R], \dot{c}_{j}\right) & \text { se } j=2, \\ \dot{c}_{j-1}\left(M_{j-1}\right)-(j-1) \sum_{\tau \in Q(j)} g_{\tau} & \text { se } 3 \leq j \leq k .\end{cases}
$$

Prova. A prova é por indução em $j$. Para $j=2$, o lema é trivial pois $M_{2}$ é uma árvore geradora mínima de $(G[R], \dot{c})$ e $\dot{c}_{2}=\dot{c}$. Suponha agora que as afirmações do lema valham para $j$ tal que $2 \leq j<k$.

Queremos mostrar que $M_{j+1}$ é uma árvore geradora mínima de $\left(G[R], \dot{c}_{j+1}\right)$ e que $\dot{c}_{j+1}\left(M_{j+1}\right)=\dot{c}_{j}\left(M_{j}\right)-j \sum_{\tau \in Q(j+1)} g_{\tau}$.

Por indução $M_{j}$ é uma árvore geradora mínima de $\left(G[R], \dot{c}_{j}\right)$. Note que $M_{j}$ é o valor de $M$ e $\dot{c}_{j}$ é o valor de $\dot{c}$ no início da iteração $j+1$ do para da linha 3 . Em cada iteração do para da linha 11 modificamos $\dot{c}$ e $M$. Reduzimos o custo de uma aresta $p(e)$ e trocamos a aresta $e$ por $p(e)$ em $M$. Por causa da redução do custo de $p(e)$ e, segundo as definições de conjunto remoção e função projeção, é fácil ver que no fim do para $M$ é uma árvore geradora mínima de $(G[R], \dot{c})$. Portanto, podemos concluir que $M_{j}$ é uma árvore geradora mínima de $\left(G[R], \dot{c}_{j}\right)$.

Note que, em cada iteração do para da linha 11, o custo de $M$ diminui de $g_{\tau}$. Logo, após o processamento de todas as arestas de $D$, o custo de $M$ diminui de $|D| g_{\tau}=(|\tau|-1) g_{\tau}$. Observe que, entre $M_{j}$ e $M_{j+1}$, são processados todos os subconjuntos de $R$ com $j+1$ terminais. Sejam $\tau_{1}, \ldots, \tau_{p}$ os subconjuntos de $R$ em $Q(j+1)$, ou seja, aqueles conjuntos cujo ganho é positivo. A diferença entre $M_{j}$ e $M_{j+1}$ é de $\left(\left|\tau_{1}\right|-1\right) g_{\tau_{1}}+\cdots+\left(\left|\tau_{p}\right|-1\right) g_{\tau_{p}}$. Como $\left|\tau_{1}\right|=\cdots=\left|\tau_{p}\right|=j+1$, temos que,

$$
\dot{c}_{j}\left(M_{j}\right)-\dot{c}_{j+1}\left(M_{j+1}\right)=j \sum_{\tau \in Q(j+1)} g_{\tau}
$$

Um outro invariante do algoritmo $B R_{k}$-avaliação é dado pelo lema abaixo.

Lema 3.2 Para qualquer subconjunto $\tau$ de $R$ tal que $|\tau| \leq j$, temos que $g\left(M_{j}, \tau\right) \leq 0$, onde $3 \leq j \leq k$.

Prova. Primeiramente, vamos mostrar que, ao fim da iteração em que $\tau$ foi processado pelo algoritmo, $g(M, \tau) \leq 0$. Depois vamos mostrar que $g(M, \tau)$ jamais cresce durante 0 algoritmo.

Seja $\tau$ um conjunto com no máximo $j$ terminais e $M$ e $\dot{c}$ no início da iteração em que $\tau$ foi processado pelo algoritmo. Se $g(M, \tau) \leq 0$, não há nada a demonstrar. Se $g(M, \tau)>0$, denote por $M^{\prime}$ e $\dot{c}^{\prime}$ os valores de $M$ e $\dot{c}$ ao final desta iteração. Seja $A$ a imagem da função $p$ definida na linha 9 do algoritmo. Como $A$ é uma árvore geradora $G[\tau]$ e as arestas de $A$ estão em $M^{\prime}$, o único conjunto remoção de $\tau$ em $M^{\prime}$ é $A$. Lembrando que 
$g\left(M^{\prime}, \tau\right)=\operatorname{ind}\left(M^{\prime}, \dot{c}^{\prime}, \tau\right)-\operatorname{smt}(G, c, \tau)$ e que $\dot{c}(A)=\operatorname{ind}\left(M^{\prime}, \dot{c}, \tau\right)$, podemos reescrever o ganho como sendo

$$
\begin{aligned}
g\left(M^{\prime}, \tau\right) & =\dot{c}(A)-\operatorname{smt}(G, c, \tau) \\
& =(\dot{c}(D)-(|\tau|-1) g(M, \tau))-\operatorname{smt}(G, c, \tau) \\
& =(\operatorname{ind}(M, \dot{c}, \tau)-(|\tau|-1) g(M, \tau))-(\operatorname{ind}(M, \dot{c}, \tau)-g(M, \tau)) \\
& =(2-|\tau|) g(M, \tau)
\end{aligned}
$$

que é negativo, pois $|\tau| \geq 3$ e $g(M, \tau)$ é positivo.

Para provar que $g(M, \tau)$ jamais cresce durante o algoritmo, basta mostrar que $g(M, \tau) \geq g\left(M^{\prime}, \tau\right)$. Note que $M$ e $M^{\prime}$ são, respectivamente, árvores geradoras mínimas de $(G[R], \dot{c})$ e $\left(G[R], \dot{c}^{\prime}\right)$. Além disso, pelas linhas 6 a 13 da fase de avaliação, temos que $M^{\prime}:=M-D+p(D)$ e, para toda aresta $x y$ em $D, \dot{c}^{\prime}(p(x y)) \leq i n d(M, \dot{c}, x y)$, onde $D$ é um conjunto remoção de $\tau$ em $M$ e $p$ a função projeção de $\bar{D}$ em $\tau$. Pelo lema 1.2 , temos que $\operatorname{ind}(M, \dot{c}, \tau) \geq \operatorname{ind}\left(M^{\prime}, \dot{c}^{\prime}, \tau\right)$. Agora, utilizando o lema 1.4 concluímos que $g(M, \tau) \geq g\left(M^{\prime}, \tau\right)$. Assim, $g(M, \tau)$ jamais cresce durante o algoritmo $B R_{k}$-avaliação.

Com isso podemos concluir a prova do lema. Para qualquer conjunto $\tau$ com no máximo $j$ terminais temos que $g\left(M_{j}, \tau\right) \leq 0$ no fim da iteração $j$ do algoritmo $B R_{k^{-}}$ avaliação.

\section{Fase de construção} $\left\{\pi_{j}\right\}_{j=3}^{k}$.

Recorde que a fase de avaliação devolve uma árvore geradora $M$ de $G[R]$ e as pilhas

A fase de construção utiliza as informações armazenadas nas pilhas para construir uma árvore de Steiner de $(G, R)$. A partir de $M$, as informações nas pilhas são processadas em ordem reversa, isto é, começamos a retirar as informações armazenadas a partir da pilha $\pi_{k}$ até $\pi_{3}$.

Inicialmente começamos com a árvore $S:=M$. A cada iteração, desempilha-se um conjunto remoção $D$, sua correspondente função projeção $p$ e um conjunto $\tau$ de terminais. Seja $A$ a imagem de $p$. Restauramos $M$, removendo $A$ e incluindo $D$. Alteramos $S$ de forma que seu custo não suba muito e, no fim da iteração, este não contenha nenhuma aresta de $A$.

Após o processamento de todas as informações das pilhas, o algoritmo devolve $S$ como solução. Veja na figura 3.2 a descrição precisa da fase de construção.

Denote por $S_{j}$ os valores de $S$ no início do para da linha 2 do algoritmo para $j=$ $k, \ldots, 3$.

Seja $c^{+}$uma função custo sobre as arestas de $S$ definida do seguinte modo: 


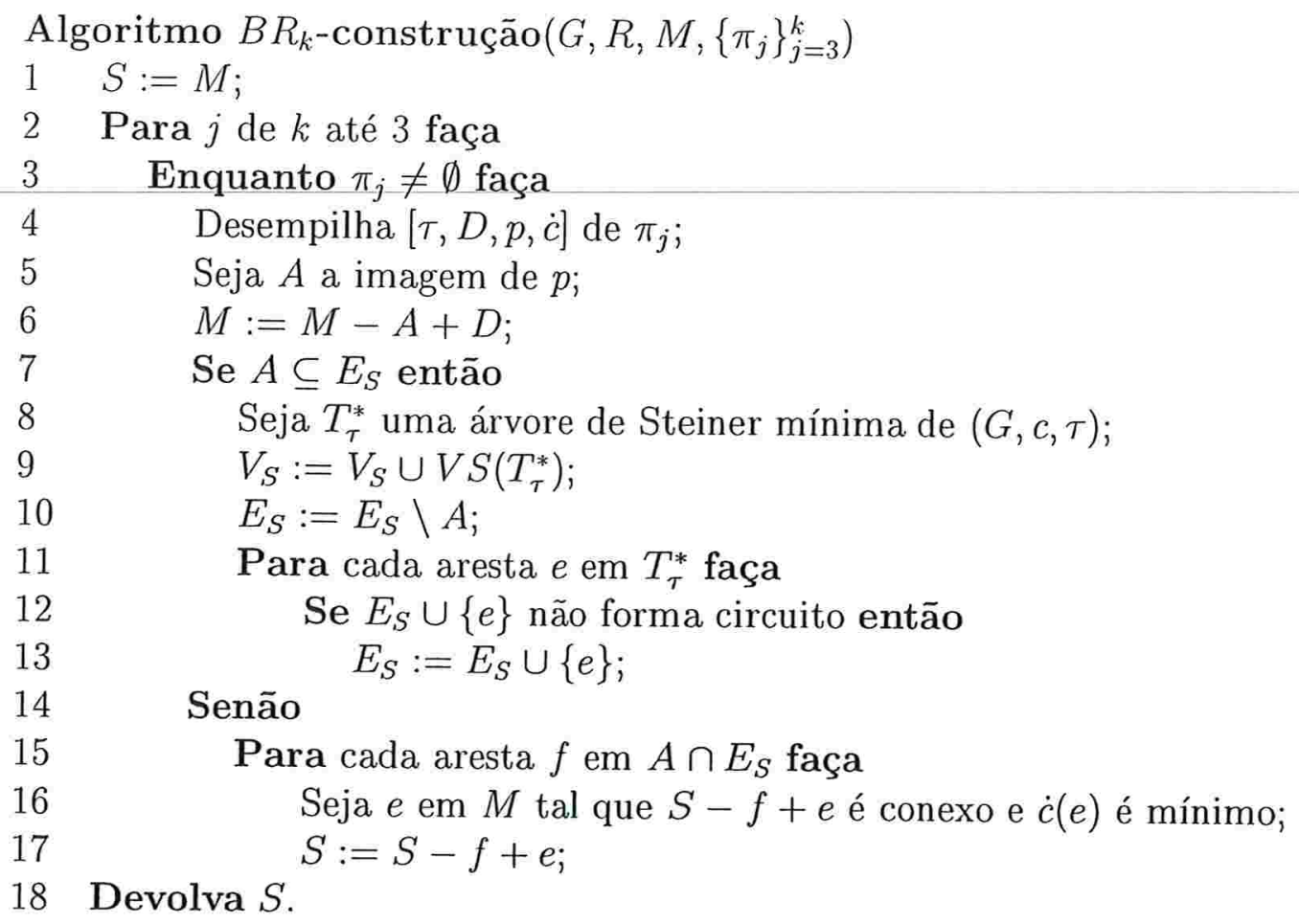

Figura 3.2: Fase de construção

$$
c^{+}(e)=\left\{\begin{array}{l}
\dot{c}(e) \quad \text { se } e \in E_{G[R]}, \\
c(e) \quad \text { caso contrário. }
\end{array}\right.
$$

Note que na linha 6 o algoritmo está desfazendo as alterações realizadas em $M$ e $\dot{c}$ na fase de avaliação. Assim, considerando que $\dot{c}$ no início da fase de construção é $\dot{c}_{k}$, os valores de $M$ e $\dot{c}$ no início do para da linha 2 são, respectivamente, $M_{j}$ e $\dot{c}_{j}$.

O seguinte invariante vale no algoritmo $B R_{k}$-construção.

Lema 3.3 Para todo $j, k \geq j \geq 2, S_{j}$ é uma árvore de Steiner de $(G, R)$ e o seu custo satisfaz o seguinte

$$
c_{j}^{+}\left(S_{j}\right) \leq \begin{cases}\dot{c}_{k}\left(M_{k}\right) & \text { se } j=k, \\ c_{j+1}^{+}\left(S_{j+1}\right)+(j-1) \sum_{\tau \in Q(j+1)} g_{\tau} & \text { se } k>j \geq 2 .\end{cases}
$$

Prova. A prova é por indução em $j$. 
Para $j=k$ as afirmações do lema são triviais pois no início do algoritmo $B R_{k}$ construção $S_{k}$ é igual a $M_{k}$. Suponha que as afirmações do lema valham para $j$ tal que $k \geq j>2$.

Vamos provar que $S_{j-1}$ é uma árvore de Steiner de $(G, R)$ e que seu custo satisfaz $c_{j-1}^{+}\left(S_{j-1}\right) \leq c_{j}^{+}\left(S_{j}\right)+(j-2) \sum_{\tau \in Q(j)} g_{\tau}$.

Para isso vamos analisar as alterações realizadas em $S$ e em seu custo no processamento de uma quádrupla $[\tau, D, p, \dot{c}]$ desempilhada.

Seja $S^{\prime}$ o valor de $S$ na linha 3 do algoritmo. Mostraremos que mesmo após as modificações de $S^{\prime}$ o grafo resultante, que chamaremos de $S^{\prime \prime}$, é uma árvore de Steiner de $(G, R)$.

Temos dois trechos do algoritmo que modificam $S^{\prime}$ (linhas 8 a 13 e 15 a 17). Mostraremos que ao término dessas modificações $S^{\prime}$ continua uma árvore de Steiner de $(G, R)$.

Seja $T_{\tau}^{*}$ uma árvore de Steiner mínima de $(G, c, \tau)$. Nas linhas 8 a 13 inserimos os vértices de Steiner de $T_{\tau}^{*}$ em $S^{\prime}$ e substituímos as arestas de $A$ em $S^{\prime}$ por arestas de $T_{\tau}^{*}$ tomando o cuidado de não deixar formar circuitos. Note que ao fim dessas instruções o grafo resultante é uma árvore.

Nas linhas 15 a 17 removemos cada aresta em $A \cap E_{S}^{\prime}$ por uma aresta $e$ em $M$ de tal modo que $S^{\prime}-f+e$ é conexo. Como $S^{\prime}$ é uma árvore então $S^{\prime}-f+e$ também é uma árvore.

Pelas observações acima e como $S^{\prime}$ é uma árvore de Steiner de $(G, R)$ pode-se concluir que após processarmos uma quádrupla o grafo $S^{\prime \prime}$ é uma árvore de Steiner de $(G, R)$.

Agora vamos analisar o que acontece $\operatorname{com} c^{+}\left(S^{\prime}\right)$ em uma iteração do enquanto na linha 3. Se todas as arestas de $A$ estão em $S^{\prime}$, substituímos $A$ por "algumas" arestas de $T_{\tau}^{*}$. Assim, o novo custo de $S^{\prime}$ é, no máximo, $c^{+}\left(S^{\prime}\right)-\dot{c}(A)+\operatorname{smt}(G, c, \tau)$. Pela definição de ganho temos que

$$
\begin{aligned}
c^{+}\left(S^{\prime}\right) & \leq c^{+}\left(S^{\prime}\right)-\left(\dot{c}(D)-(|\tau|-1) g_{\tau}\right)+\left(\dot{c}(D)-g_{\tau}\right) \\
& \leq c^{+}\left(S^{\prime}\right)+(|\tau|-1) g_{\tau}-g_{\tau} \\
& \leq c^{+}\left(S^{\prime}\right)+(|\tau|-2) g_{\tau} .
\end{aligned}
$$

Agora vamos analisar o caso em que nem todas as arestas de $A$ estão em $S^{\prime}$. Nas linhas 13 a 15 do algoritmo, substitui-se todas as arestas que estão em $A$ e em $S^{\prime}$ por arestas de $M$. Seja $f:=u v$ uma dessas arestas, onde $u, v \in \tau$. Pela fase de avaliação, $\dot{c}(f)=\operatorname{ind}(M, \dot{c}, u v)-g_{\tau}$. Seja $e$ a aresta em $M$ escolhida na linha 14 . Note que $e$ está no caminho em $M$ entre $u$ e $v$, logo $\dot{c}(e) \leq \operatorname{ind}(M, \dot{c}, u v)$. Portanto $\dot{c}(e)-\dot{c}(f) \leq$ $\dot{c}(e)-i n d(M, \dot{c}, u v) \leq g_{\tau}$. Como $\left|A \cap E_{S}^{\prime}\right| \leq|\tau|-2$, então após processarmos todas as arestas, o custo de $S^{\prime}$ aumenta de no máximo $(|\tau|-2) g_{\tau}$.

Portanto, em ambos os casos, o custo de $S^{\prime}$ aumenta de no máximo $(|\tau|-2) g_{\tau}$ para cada quádrupla desempilhada.

Agora estamos prontos para provar que o lema é verdadeiro para $j-1$. Observe que, entre $S_{j}$ e $S_{j-1}$, são processadas todas as quádruplas armazenadas em $\pi_{j-1}$. 
Logo, a diferença entre os custos de $S_{j-1}$ e $S_{j}$ é no máximo o somatório dos aumentos no custo de $S$ para cada quádrupla analisada. Lembrando que $Q(j-1)$ é o conjunto de todos os $\tau$ armazenados em $\pi_{j-1}$, temos que $c_{j-1}^{+}\left(S_{j-1}\right)-c_{j}^{+}\left(S_{j}\right) \leq(j-2) \sum_{\tau \in Q(j)} g_{\tau}$.

O algoritmo de Berman e Ramayer consiste da chamada dos algoritmos $B R_{k}$-avaliação e $B R_{k}$-construção. Pelo lema 3.3 é fácil ver que a solução produzida pelo algoritmo é uma árvore de Steiner em $(G, R)$ além disso as duas fases do algoritmo de Berman e Ramayer podem ser implementadas de forma que o consumo de tempo delas seja polinomial.

\subsection{Análise do algoritmo de Berman e Ramayer}

Sejam $m_{j}$ e $s_{j}$ os valores de $\dot{c}_{j}\left(M_{j}\right)$ e $c_{j}^{+}\left(S_{j}\right)$ nos algoritmos $B R_{k^{-}}$avaliação e $B R_{k^{-}}$ construção respectivamente, para $j=2, \ldots, k$.

Lema 3.4 Sejam $m_{j}$ o valor de $M_{j}$ na fase de avaliação e $t_{j}$ o valor de uma árvore $j$-restrita minima $T_{j}^{*}$ de $(G, c, R)$. Então $m_{j} \leq t_{j}$ para $j=2, \ldots, k$.

Prova. Sejam $\tau_{1}, \ldots, \tau_{l}$ o conjunto de terminais de cada um dos $l$ componentes cheios de $T_{j}^{*}$. Podemos escrever o custo $m_{j}$ como

$$
\begin{aligned}
m_{j} & =\operatorname{mst}\left(G[R], \dot{c}_{j}\right)-\operatorname{mst}\left(G[R], \dot{c}_{j}\left[\tau_{1}, \ldots, \tau_{l}\right]\right) \\
& =\sum_{i=1}^{l}\left(\operatorname{mst}\left(G[R], \dot{c}_{j}\left[\tau_{1}, \ldots, \tau_{i-1}\right]\right)-\operatorname{mst}\left(G[R], \dot{c}_{j}\left[\tau_{1}, \ldots, \tau_{i}\right]\right)\right) \\
& \leq \sum_{i=1}^{l}\left(\operatorname{mst}\left(G[R], \dot{c}_{j}\right)-\operatorname{mst}\left(G[R], \dot{c}_{j}\left[\tau_{i}\right]\right)\right) \\
& =\sum_{i=1}^{l}\left(g\left(M_{j}, \tau_{i}\right)+\operatorname{smt}\left(G, c, \tau_{i}\right)\right) \\
& \leq \sum_{i=1}^{l} \operatorname{smt}\left(G, c, \tau_{i}\right)=c\left(T_{j}^{*}\right)=t_{j} .
\end{aligned}
$$

A desigualdade (3.1) vale pelo corolário 1.3 e pela equação (1.1), e a desigualdade (3.2) vale pelo lema 3.2 , que garante que $g\left(M_{j}, \tau_{i}\right) \leq 0$.

A seguir mostramos a prova de uma razão de aproximação para o algoritmo.

Teorema 3.5 O algoritmo de Berman e Ramayer tem uma razão de aproximação de, no máximo,

$$
r_{2}-\sum_{i=3}^{k} \frac{r_{i-1}-r_{i}}{i-1}
$$

onde $r_{k}:=\sup \left\{\frac{\operatorname{srnt}_{k}(G, c, R)}{\operatorname{smt}(G, c, R)}\right\}$. 
Prova. Pelos lemas 3.1 e 3.3 , temos que

$$
\begin{aligned}
s_{j-1}-s_{j} & \leq(j-2) \sum_{\tau \in Q(j)} g_{\tau} \quad \text { para } j=k, \ldots, 3 \mathrm{e} \\
m_{j-1}-m_{j} & =(j-1) \sum_{\tau \in Q(j)} g_{\tau} \quad \text { para } j=3, \ldots, k .
\end{aligned}
$$

Substituindo a segunda expressão na primeira obtemos que, para $j=3, \ldots, k$,

$$
\begin{aligned}
s_{j-1}-s_{j} & \leq \frac{j-2}{j-1}\left(m_{j-1}-m_{j}\right) \\
& =\left(m_{j-1}-m_{j}\right)-\frac{1}{j-1}\left(m_{j-1}-m_{j}\right) .
\end{aligned}
$$

Somando-se para todo $j$, obtemos

$$
s_{2}-s_{k} \leq m_{2}-m_{k}-\sum_{j=3}^{k} \frac{1}{j-1}\left(m_{j-1}-m_{j}\right)
$$

e como $S_{k}=M_{k}$, concluímos que

$$
\begin{aligned}
s_{2} & \leq m_{2}-\sum_{j=3}^{k} \frac{1}{j-1}\left(m_{j-1}-m_{j}\right) \\
& =\sum_{j=2}^{k-1} m_{j}\left(\frac{1}{j-1}-\frac{1}{j}\right)+\frac{1}{k-1} m_{k} .
\end{aligned}
$$

Agora, considere uma árvore $j$-restrita mínima $T_{j}$ de $(G, c, R)$ e seja $t_{j}$ o seu custo. Pelo lema $3.4, m_{j} \leq t_{j}$ para $2 \leq j \leq k$. Então, usando a equação (3.4), deduzimos que

$s_{2} \leq \sum_{j=2}^{k-1} m_{j} \underbrace{\left(\frac{1}{j-1}-\frac{1}{j}\right)}_{\geq 0}+\frac{1}{k-1} m_{k} \leq \sum_{j=2}^{k-1} t_{j}\left(\frac{1}{j-1}-\frac{1}{j}\right)+\frac{1}{k-1} t_{k}=t_{2}-\sum_{j=3}^{k} \frac{t_{j-1}-t_{j}}{j-1}$.

Dividindo tudo pelo custo de uma árvore de Steiner mínima de $(G, R, c)$, obtemos

$$
\frac{s_{2}}{\operatorname{smt}(G, c, R)} \leq \frac{t_{2}}{\operatorname{smt}(G, c, R)}-\sum_{j=3}^{k} \frac{t_{j-1}-t_{j}}{\operatorname{smt}(G, c, R)(j-1)}=r_{2}-\sum_{j=3}^{k} \frac{r_{j-1}-r_{j}}{j-1}
$$




\subsection{Comparação com o algoritmo das árvores 3 - res- tritas}

Se, na fase-de avaliação do-algoritmo de Berman e Ramayer para $k=3$, escolhemos as triplas em ordem do ganho - as de ganho maior primeiro - então o algoritmo de Berman e Ramayer reduz-se ao algoritmo das árvores 3-restritas.

O lema 2.2 no fundo garante que apenas as triplas escolhidas pelo algoritmo das árvores 3-restritas são empilhadas e que, na fase de construção, a condição da linha 7 está sempre satisfeita, ou seja, uma árvore de Steiner de uma tripla empilhada na fase de avaliação será incluída na árvore final. 


\section{Capítulo 4}

\section{Algoritmo do ganho relativo}

Os algoritmos das árvores 3-restritas e o de Berman e Ramayer utilizam a função ganho para escolher árvores $k$-restritas mínimas, onde $k=3$ para o primeiro algoritmo e $k$ é uma constante fixa maior ou igual a 3 para o segundo. Os vértices de Steiner dessas árvores escolhidas compõem, junto com os terminais, uma árvore de Steiner produzida por esses dois algoritmos como solução do PSG.

O algoritmo do ganho relativo, também proposto por Zelikovsky [49], é guloso e utiliza a mesma idéia de escolher árvores $k$-restritas mínimas para melhorar o custo da solução corrente. A diferença fundamental entre este algoritmo e os anteriores está no processo de escolha das árvores $k$-restritas.

Novamente, o algoritmo que apresentaremos consiste na verdade em uma família de algoritmos - um algoritmo para cada valor de $k$. Assim, assumimos no restante do capítulo que $k$ é uma constante fixa maior ou igual a 3 .

\subsection{Ganho relativo}

Sejam $G$ um grafo completo, $c$ uma função de $E_{G}$ em $Q_{>0}$ satisfazendo a desigualdade triangular e $R$ um conjunto de vértices - os terminais. Considere também a restrição $\dot{c}$ de $c$ a $G[R]$, uma árvore geradora mínima $M$ de $(G[R], \dot{c})$ e um subconjunto $\tau$ de $R$. O ganho relativo de $\tau$ em $M$, denotado por $g_{r}(M, \tau)$, é a razão entre o custo de uma árvore de Steiner mínima de $(G, c, \tau)$ e o custo de um conjunto remoção de $\tau$ em $M$. Em fórmula,

$$
g_{r}(M, \tau):=\frac{\operatorname{smt}(G, c, \tau)}{\operatorname{ind}(M, \dot{c}, \tau)} .
$$

Note que ficou implícita a dependência do ganho relativo das funções $c$ e $\dot{c}$.

\subsection{Descrição do algoritmo}

A entrada do algoritmo é um grafo completo $G$, uma função $c$ de $E_{G}$ em $Q_{>0}$ sa- 
tisfazendo a desigualdade triangular e um conjunto $R$ de vértices de $G$. Como saída, o algoritmo produz uma árvore de Steiner de $(G, R)$ cujo custo dividido pelo custo de uma solução ótima do PSG para $(G, c, R)$ é no máximo $\left(1+\ln \left(\frac{r_{2}}{r_{k}}\right)\right) r_{k}$. Quando $k$ cresce, essa delimitação se aproxima de $1+\ln \left(r_{2}\right)=1+\ln (2)<1,694$.

Seja $\dot{c}$ a restrição de $c$ a $G[R]$ e, para um conjunto $\tau$ de no máximo $k$ terminais, denote por $T_{\tau}$ uma árvore de Steiner mínima de $(G, c, \tau)$.

$\mathrm{O}$ algoritmo começa com uma árvore geradora mínima $M$ de $(G[R], \dot{c})$ e um conjunto $W$ de vértices, inicialmente vazio. Em cada iteração, o algoritmo escolhe um conjunto $\tau$ e constrói $T_{\tau}$ e um conjunto remoção $D$ de $\tau$ em $M$ que minimiza $g_{r}(M, \tau)$. O algoritmo adiciona os vértices de Steiner de $T_{\tau}$ a $W$ e zera o custo $\dot{c}(e)$ de cada aresta $e$ em $G[R]$ que está na imagem da função projeção de $D$ em $\tau$. Além disso, o algoritmo altera $M$ de forma a mantê-la uma árvore geradora mínima de $(G[R], \dot{c})$. O algoritmo pára quando o custo de $M$ é igual a zero. Neste ponto o algoritmo devolve uma árvore geradora mínima em $(G[R \cup W], c)$. Na figura 4.1 está a descrição precisa do algoritmo do ganho relativo.

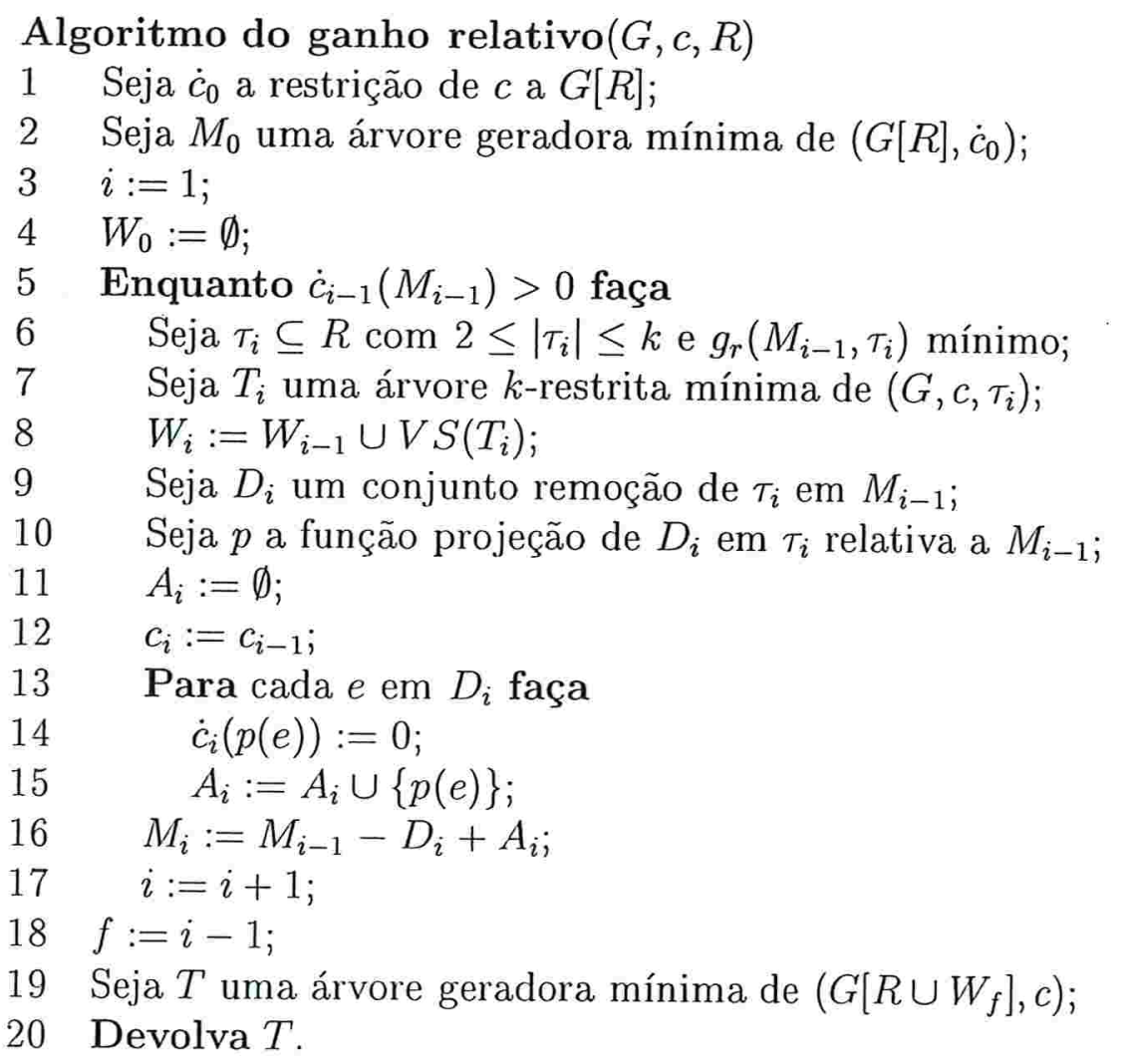

Figura 4.1: Algoritmo do ganho relativo 
Note que

$$
g_{r}\left(M_{i-1}, \tau_{i}\right):=\frac{\operatorname{smt}\left(G, c, \tau_{i}\right)}{\operatorname{ind}\left(M_{i-1}, \dot{c}_{i-1}, \tau_{i}\right)} \leq 1,
$$

pois existe $\tau$ tal que $g_{r}\left(M_{i-1}, \tau\right)=1$. Basta tomar como $\tau$ os extremos de uma aresta de $M_{0} \cap M_{i-1}$. (Se não há nenhuma aresta em $M_{0} \cap M_{i-1}$, então o custo de $M_{i-1}$ é nulo e o algoritmo teria parado.) Além disso, note que o algoritmo do ganho relativo pode ser implementado de forma que seu consumo de tempo seja polinomial no tamanho da sua entrada.

\subsection{Análise do algoritmo do ganho relativo}

O lema a seguir mostra a relação entre a árvore devolvida como solução pelo algoritmo e as árvores de Steiner escolhidas durante o algoritmo.

Lema 4.1 Seja $T$ a solução produzida pelo algoritmo do ganho relativo e $T_{1}, \ldots, T_{f}$ as árvores de Steiner escolhidas. Temos que

$$
c(T) \leq \sum_{j=1}^{f} c\left(T_{j}\right) .
$$

Prova. Seja $T^{\prime}:=\bigcup_{j=1}^{f} T_{j}$. Para provar o lema é suficiente mostrar que $T^{\prime}$ é conexo e que seu conjunto de vértices é igual ao conjunto de vértices de $T$.

Suponha por absurdo que $T^{\prime}$ não é conexo. Logo existem pelo menos dois componentes $A$ e $B$ em $T^{\prime}$. Como $T^{\prime}$ é formado pela união de $T_{j}$ para $j=1, \ldots, f$ então em cada um dos componentes $A$ e $B$ existe pelo menos um terminal. Seja $x y$ uma aresta de $M_{0}$ tal que $x$ está em $A$ e $y$ está em $B$. Note que não existe nenhum $T_{j}, j=1, \ldots, f$, que contenha $x$ e $y$, logo $x y$ está em $M_{f}$ e $g_{r}\left(M_{f},\{x, y\}\right)=1$. Portanto $c_{f}(x y)=c_{0}(x y)>0$ contrariando a condição de parada do algoritmo. Assim, podemos concluir que $T^{\prime}$ é conexo.

A união dos vértices de Steiner das árvores de Steiner escolhidas pelo algoritmo é igual a $W_{f}$. Suponha por absurdo que existe um vértice $v$ em $R$ tal que $v$ não está em $T^{\prime}$. Note que existe uma aresta $u v$ em $M_{0}$ para algum $u$ em $R$ e tal aresta está em $M_{f}$ já que $v$ não está em nenhum $T_{j}$. Além disso $\dot{c}_{f}(u v)=\dot{c}_{0}(u v)>0$, ou seja, $\dot{c}\left(M_{f}\right)>0$, uma contradição. Logo o conjunto de vértices de $T$ é igual ao conjunto de vértices de $T^{\prime}$.

O próximo lema mostra um delimitador superior da razão entre o custo da solução produzida pelo algoritmo do ganho relativo e do custo de uma árvore $k$-restrita mínima de $(G, c, R)$.

Lema 4.2 Sejam $G$ um grafo completo, c uma função de $E_{G}$ em $Q_{>0}$ satisfazendo a desigualdade triangular e $R$ um conjunto de vértices de $G$. O custo da solução do algoritmo 
do ganho relativo sobre o custo de uma árvore $k$-restrita mínima de $(G, c, R)$ é no máximo $\left(1+\ln \frac{r_{2}}{r_{k}}\right)$.

Prova. Seja $T^{*}$ uma árvore $k$-restrita mínima em $(G, c, R)$ e, para cada componente cheio $T_{j}^{*}$ de $T^{*}$, considere $\tau_{j}^{*}$ os vértices terminais de $T_{j}^{*}$ para $1 \leq j \leq l$.

Em cada iteração, o algoritmo escolhe um conjunto $\tau_{i}$ de no máximo $k$ terminais que minimiza o ganho relativo. Assim,

$$
g_{r}\left(M_{i-1}, \tau_{i}\right) \leq \min _{j}\left\{g_{r}\left(M_{i-1}, \tau_{j}^{*}\right)\right\} .
$$

Sabemos que, para números não-negativos $a_{j}$ e $b_{j}, 1 \leq j \leq l$,

$$
\min _{j} \frac{a_{j}}{b_{j}} \leq \frac{\sum_{j} a_{j}}{\sum_{j} b_{j}}
$$

Tomando $a_{j}=c\left(T_{j}^{*}\right)$ e $b_{j}=\operatorname{ind}\left(M_{i-1}, \dot{c}_{i-1}, \tau_{j}^{*}\right)$ e, utilizando (4.1) e (4.3), temos que

$$
g_{r}\left(M_{i-1}, \tau_{i}\right) \leq \min _{j}\left\{\frac{c\left(T_{j}^{*}\right)}{\operatorname{ind}\left(M_{i-1}, \dot{c}_{i-1}, \tau_{j}^{*}\right)}\right\} \leq \frac{\sum_{j} c\left(T_{j}^{*}\right)}{\sum_{j} \operatorname{ind}\left(M_{i-1}, \dot{c}_{i-1}, \tau_{j}^{*}\right)} .
$$

Pelo corolário 1.3, o denominador do lado direito da desigualdade acima pode ser substituído por

$$
\sum_{j=1}^{l} i n d\left(M_{j}^{\prime}, \dot{c}_{i-1}\left[\tau_{1}^{*}, \ldots, \tau_{j-1}^{*}\right], \tau_{j}^{*}\right)
$$

onde $M_{j}^{\prime}$ é uma árvore geradora mínima de $\left(G[R], \dot{c}_{i-1}\left[\tau_{1}^{*}, \ldots, \tau_{j-1}^{*}\right]\right)$ e que, pela definição de índice, é uma soma telescópica. Assim

$$
\begin{aligned}
g_{r}\left(M_{i-1}, \tau_{i}\right) & \leq \frac{\operatorname{smt}_{k}(G, c, R)}{m s t\left(G[R], \dot{c}_{i-1}\right)-m s t\left(G[R], \dot{c}_{i-1}\left[\tau_{1}^{*}, \ldots, \tau_{l}^{*}\right]\right)} \\
& =\frac{\operatorname{smt}_{k}(G, c, R)}{\operatorname{mst}\left(G[R], \dot{c}_{i-1}\right)},
\end{aligned}
$$

já que $\operatorname{mst}\left(G[R], \dot{c}_{i-1}\left[\tau_{1}^{*}, \ldots, \tau_{l}^{*}\right]\right)=0$ pois $\bigcup_{j=1}^{l} T_{j}^{*}=T^{*}$ e conecta $R$.

Sejam $m_{i}:=\operatorname{mst}\left(G[R], \dot{c}_{i}\right), \operatorname{smt}_{k}:=\operatorname{smt}_{k}(G, c, R)$ e $\operatorname{smt}:=\operatorname{smt}(G, c, R)$. Usando a definição de ganho relativo, temos que

$$
g_{r}\left(M_{i-1}, \tau_{i}\right)=\frac{c\left(T_{i}\right)}{\operatorname{ind}\left(M_{i-1}, \dot{c}_{i-1}, \tau_{i}\right)}=\frac{c\left(T_{i}\right)}{m_{i-1}-m_{i}} .
$$

Portanto, aplicando as desigualdades (4.2) e (4.5),

$$
\begin{aligned}
\sum_{i=1}^{f} c\left(T_{i}\right) & =\sum_{i=1}^{f} g_{r}\left(M_{i-1}, \tau_{i}\right)\left(m_{i-1}-m_{i}\right) \\
& \leq \sum_{i=1}^{f} \min \left\{1, \frac{s m_{k}}{m_{i-1}}\right\}\left(m_{i-1}-m_{i}\right) .
\end{aligned}
$$


Note que a seqüência $m_{0}, m_{1}, \ldots, m_{f}$, que são os custos das árvores geradoras mínimas em cada iteração do algoritmo, é monotonicamente decrescente, sendo que $m_{f}=0$ e $m_{0}=m s t(G[R], \dot{c})$. Assim, estimamos o somatório em (4.6) por uma integral do seguinte modo

$$
\begin{aligned}
\sum_{i=1}^{f} \min \left\{1, \frac{s m t_{k}}{m_{i-1}}\right\}\left(m_{i-1}-m_{i}\right) & \leq \sum_{i=1}^{f} \int_{m_{i}}^{m_{i-1}} \min \left\{1, \frac{s m t_{k}}{x}\right\} d x \\
& \leq \int_{m_{f}}^{m_{0}} \min \left\{1, \frac{s m t_{k}}{x}\right\} d x \\
& =\int_{0}^{s m t_{k}} d x+s m t_{k} \int_{s m t_{k}}^{m_{0}} \frac{1}{x} d x \\
& =s m t_{k}+s m t_{k}\left(\ln \frac{m_{0}}{s m t_{k}}\right) \\
& =s m t_{k}\left(1+\ln \frac{m_{0}}{s m t} \frac{s m t}{s m t_{k}}\right) \\
& =s m t_{k}\left(1+\ln \frac{r_{2}}{r_{k}}\right) .
\end{aligned}
$$

Do lema 4.1 e das desigualdades (4.6) e (4.7), temos que

$$
c(T) \leq \sum_{i=1}^{f} c\left(T_{i}\right) \leq\left(1+\ln \frac{r_{2}}{r_{k}}\right) s m t_{k}(G, c, R) .
$$

Abaixo segue a prova de uma razão de aproximação para o algoritmo do ganho relativo.

Teorema 4.3 Sejam $G$ um grafo completo, c uma função de $E_{G}$ em $Q_{>0}$ satisfazendo a desigualdade triangular e $R$ um conjunto de vértices de $G$. O custo de uma solução do algoritmo do ganho relativo para $(G, c, R)$ sobre o custo de uma árvore de Steiner mínima de $(G, c, R)$ é no máximo $\left(1+\ln \frac{r_{2}}{r_{k}}\right) r_{k}$.

Prova. Seja $T$ a solução produzida pelo algoritmo do ganho relativo. Pelo lema 4.2, temos que

$$
\frac{c(T)}{s m t(G, c, R)}=\frac{c(T)}{s m t_{k}(G, c, R)} \cdot \frac{s m t_{k}(G, c, R)}{s m t(G, c, R)}=\frac{c(T)}{s m t_{k}(G, c, R)} r_{k} \leq\left(1+\ln \frac{r_{2}}{r_{k}}\right) r_{k} .
$$

Para $k$ suficientemente grande, a delimitação acima se aproxima de $1+\ln 2<1,694$.

Similarmente ao algoritmo de Berman e Ramayer, não se conhece uma instância do PSG que resulte exatamente na razão de aproximação demonstrada para o algoritmo do ganho relativo. A instância que resultou na maior razão de aproximação conhecida para o algoritmo do ganho relativo foi exibida por Gröpl, Hougardy, Nierhoff e Prömel [22]. A razão de aproximação obtida foi de 1,33 para $k$ suficientemente grande. 


\section{Capítulo 5}

\section{Algoritmo do pré-processamento}

Com exceção do algoritmo de Berman e Ramayer, os algoritmos das árvores 3-restritas e do ganho relativo são gulosos. Em cada iteração eles escolhem um conjunto $\tau$ de no máximo $k$ terminais e uma árvore de Steiner sobre $\tau$ que sejam ótimos segundo algum critério de escolha e acrescentam os vértices de Steiner dessa árvore a um conjunto $W$. Se considerarmos o valor de $\operatorname{smt}(G, c, R \cup W)$ durante a execução de cada um desses algoritmos, esse número pode aumentar quando o algoritmo escolhe um vértice de Steiner "errado" para incluir em $W$.

Karpinski e Zelikovsky [31] apresentam uma maneira de levar em conta esse aumento. O algoritmo proposto por eles utiliza o que chamaremos de floresta de terminais mínima. Além disso, o algoritmo do pré-processamento também depende de um parâmetro fixo $k$ que delimita o número de terminais das árvores $k$-restritas que o algoritmo seleciona.

\subsection{Floresta de terminais e ganho ponderado}

Sejam $G$ um grafo e $R$ um conjunto de vértices de $G$ - os terminais. Seja $T$ uma árvore de Steiner de $(G, R)$. Lembrando que $V S(T)$ é o conjunto de vértices de Steiner de $T$, um subgrafo $F$ de $T$ é uma floresta de terminais de $(T, R)$ se, para qualquer $v$ em $V S(T)$, existe um caminho em $F$ que conecta $v$ a algum vértice de $R$. Veja a figura 5.1.

Uma floresta de terminais mínima $F^{*}$ de $(T, c, R)$ é uma floresta de terminais tal que, para qualquer floresta de terminais $F$ de $(T, R)$, temos que $c\left(F^{*}\right) \leq c(F)$. O custo de $F^{*}$ é o que chamamos de perda de $(T, c, R)$ e denotamos por $\operatorname{perda}(T, c, R)$. Note que $\operatorname{perda}(T, c, R)=\sum_{i} \operatorname{perda}\left(T_{i}, c, \tau_{i}\right)$, onde $T_{i}$ é um componente cheio de $T$ e $\tau_{i}:=V T\left(T_{i}\right)$.

O lema seguinte nos dá um importante limitante superior para a perda de uma árvore de Steiner.

Lema 5.1 Para qualquer árvore de Steiner $T$ de $(G, c, R)$, cujos vértices de Steiner têm grau pelo menos 3 , temos que

$$
\operatorname{perda}(T, c, R) \leq \frac{1}{2} c(T)
$$




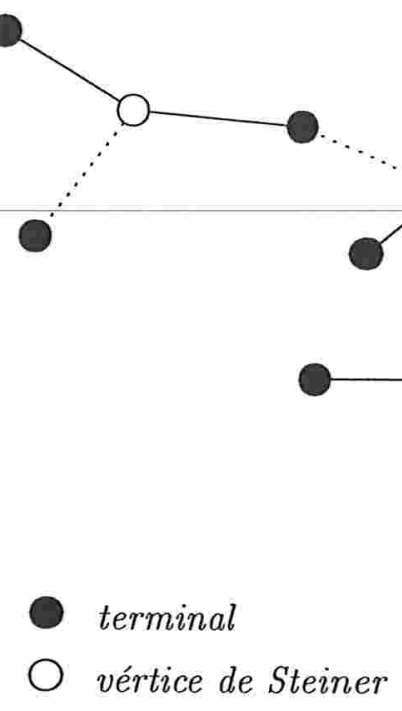

Figura 5.1: Uma árvore de Steiner onde as linhas pontilhadas representam uma floresta de terminais da árvore.

Prova. Para provar este lema, basta mostrar que para qualquer componente cheio $T_{\tau}$ de $T$ vale $\operatorname{perda}\left(T_{\tau}, c, \tau\right) \leq \frac{1}{2} c\left(T_{\tau}\right)$, onde $\tau=V T\left(T_{\tau}\right)$.

Seja $r$ um terminal de $T_{\tau}$. Oriente as arestas de $T_{\tau}$ de forma que $T_{\tau}$ torne-se uma árvore enraizada em $r$.

Agora vamos construir uma floresta de terminais. Para cada vértice de Steiner $s$, escolha uma das arestas de custo mínimo, digamos $f$, entrando em $s$ (note que todo vértice de Steiner tem pelo menos uma aresta entrando nele). Seja $F$ o conjunto das arestas escolhidas - $F$ é uma floresta de terminais. De fato, todo vértice de Steiner está conectado em $F$ a uma folha da árvore, que é um terminal. O custo de $F$ é não mais que $\frac{1}{2} c\left(T_{\tau}\right)$ pois, para cada aresta e em $F$, há pelo menos uma aresta de $T_{\tau}$ fora de $F$ de custo pelo menos $c(e)$, já que cada vértice de Steiner em $T_{\tau}$ tem grau pelo menos 3 . Veja a figura 5.2 .

Note que toda árvore de Steiner $T$ pode ser transformada em uma árvore de Steiner com custo no máximo $c(T)$ onde cada vértice de Steiner tem grau no mínimo três. A primeira observação é que podemos remover de $T$ os vértices de Steiner de grau zero ou um. Se um vértice de Steiner $v$ tem grau dois, exclua $v$ e as arestas incidentes a $v$ de $T$ e insira a aresta em $G$ que conecta os dois vértices adjacentes a $v$ em $T$. Por causa da desigualdade triangular, se denotarmos por $T^{\prime}$ a árvore resultante, temos que $c\left(T^{\prime}\right) \leq c(T)$.

Sejam $G$ um grafo completo, $c$ uma função de $E_{G}$ em $Q_{>0}$ satisfazendo a desigualdade triangular e $R$ um conjunto de vértices - os terminais. Dados uma função $\dot{c}$ de $E_{G[R]}$ em $Q_{>0}$, uma árvore geradora mínima $M$ de $(G[R], \dot{c})$ e um conjunto $\tau$ de terminais, o 


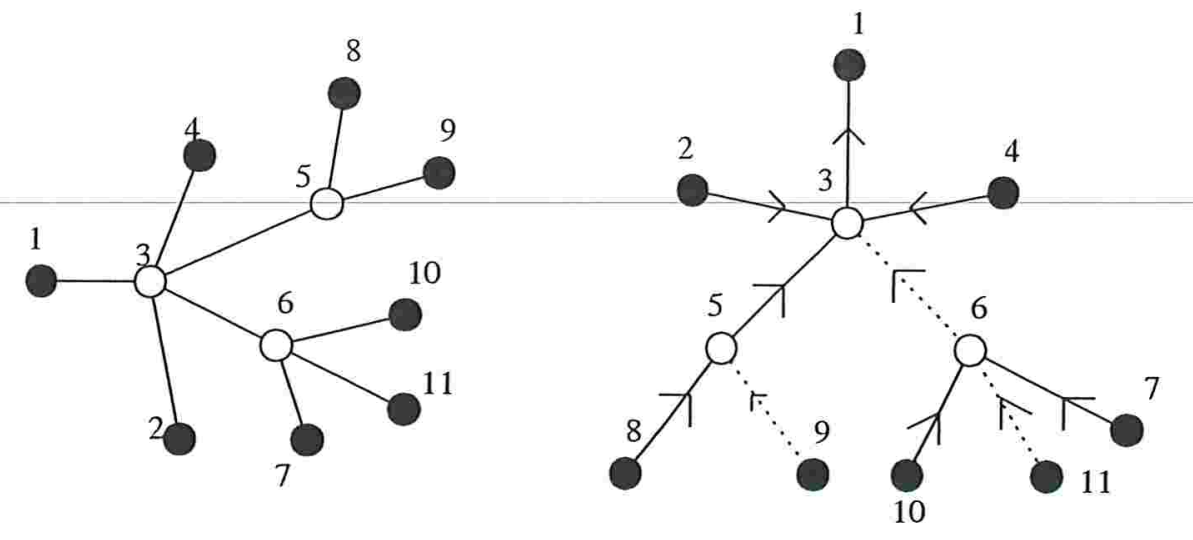

(a)

(b)

terminal

- vértice de Steiner

Figura 5.2: (a) Um componente cheio $T_{\tau}$ de $T$. (b) Uma árvore enraizada $T_{\tau}$ com raiz no vértice 1 . Linhas pontilhadas representam arestas da floresta de terminais de $T_{\tau}$.

ganho ponderado de $\tau$ em $M$, denotado por $g_{p}(M, \tau)$, é dado por

$$
g_{p}(M, \tau):=\min _{T_{\tau}}\left\{\frac{c\left(T_{\tau}\right)+\frac{1}{2} \operatorname{perda}\left(T_{\tau}, c, \tau\right)}{\operatorname{ind}(M, \dot{c}, \tau)}\right\},
$$

onde o mínimo é tomado dentre todas as árvores $k$-restritas $T_{\tau}$ de $(G, \tau)$.

Note que está implícita a dependência do ganho ponderado das funções $c$ e $\dot{c}$.

\subsection{Descrição do algoritmo}

A entrada do algoritmo é um grafo completo $G$, uma função $c$ de $E_{G}$ em $Q_{>0}$ satisfazendo a desigualdade triangular e um conjunto $R$ de vértices de $G$. Como saída, o algoritmo produz uma árvore de Steiner de $(G, R)$ cujo custo dividido pelo custo de uma solução ótima do PSG, para $k$ suficientemente grande, é no máximo 1,645.

Seja $\dot{c}$ a restrição de $c$ a $G[R]$. O algoritmo começa com uma árvore geradora mínima $M$ de $(G[R], \dot{c})$ e um conjunto $W$ de vértices, inicialmente vazio. Em cada iteração, o algoritmo escolhe uma árvore $k$-restrita de $(G, c, \tau) \operatorname{com} \tau \subseteq R$ e $|\tau| \leq k$ (note que $k$ está fixo durante o algoritmo) que minimize $g_{p}(M, \tau)$ e constrói um conjunto remoção $D_{\tau}$ de $\tau$ em $M$. O algoritmo adiciona os vértices de Steiner de $T_{\tau}$ a $W$ e zera o custo $\dot{c}(e)$ de cada aresta e em $G[R]$ que está na imagem da função projeção de $D_{\tau}$ em $\tau$. Além disso, o algoritmo altera $M$ de forma a mantê-la uma árvore geradora mínima de $(G[R], \dot{c})$. O 
algoritmo pára quando $\dot{c}(M)=0$. Neste ponto, o algoritmo executa o algoritmo do ganho relativo para $(G, c, R \cup W)$. Na figura 5.3 , está a descrição precisa do algoritmo do pré-processamento.

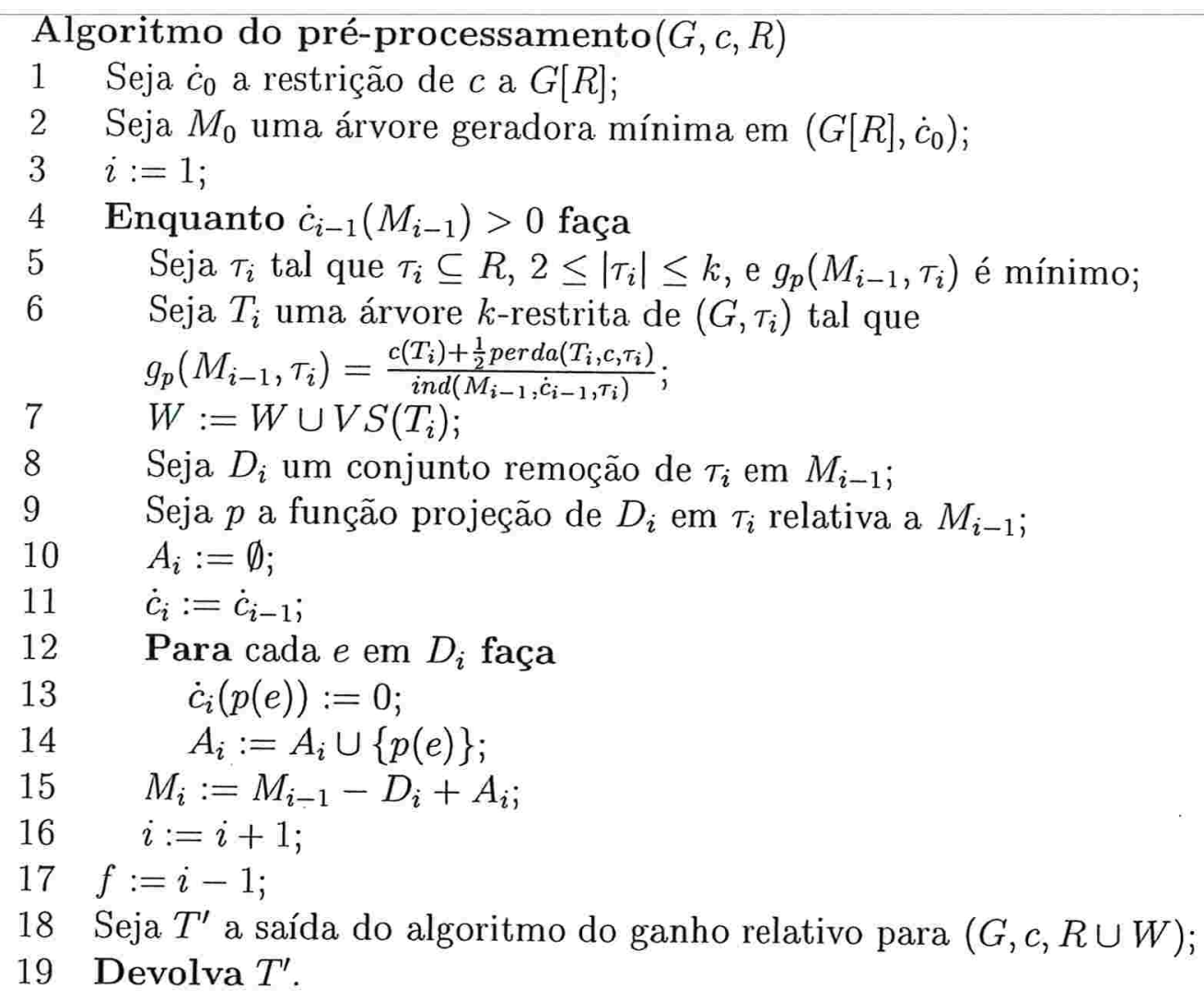

Figura 5.3: Algoritmo do pré-processamento

Note que o algoritmo do pré-processamento pode ser implementado de forma que seu consumo de tempo seja polinomial.

Como

$$
\frac{c\left(T_{\tau}\right)+\frac{1}{2} \operatorname{perda}\left(T_{\tau}, c, \tau\right)}{\operatorname{ind}\left(M_{i-1}, \dot{c}_{i-1}, \tau\right)}=1
$$

quando $\tau$ é o conjunto dos extremos de uma aresta de $M_{i-1}$ com custo diferente de zero e $T_{\tau}$ é uma árvore $k$-restrita de $(G, \tau)$, temos que

$$
g_{p}\left(M_{i-1}, \tau_{i}\right)=\frac{c\left(T_{i}\right)+\frac{1}{2} \operatorname{perda}\left(T_{i}, c, \tau_{i}\right)}{\operatorname{ind}\left(M_{i-1}, \dot{c}_{i-1}, \tau_{i}\right)} \leq 1 .
$$




\subsection{Análise do algoritmo do pré-processamento}

O algoritmo será analisado em duas fases. A primeira fase compreende as linhas 1 a 17 do algoritmo e a segunda fase corresponde à execução do algoritmo do ganho relativo (linha 18). O seguinte lema corresponde à análise da primeira fase.

Lema 5.2 Sejam $G$ um grafo completo, c uma função de $E_{G}$ em $Q_{>0}$ satisfazendo a desigualdade triangular e $R$ um conjunto de vértices de $G$. Se $T_{1}, \ldots, T_{f}$ são as árvores $k$ restritas escolhidas pelo algoritmo do pré-processamento para $(G, c, R)$ e $\tau_{1}, \ldots, \tau_{f}$ seus respectivos conjuntos de terminais, então

$$
\sum_{i=1}^{f}\left(c\left(T_{i}\right)+\frac{1}{2} \operatorname{perda}\left(T_{i}, c, \tau_{i}\right)\right) \leq \frac{5}{4} \operatorname{smt}_{k}(G, c, R)\left(1+\ln \frac{4}{5} \frac{m s t(G[R], c)}{s m t_{k}(G, c, R)}\right) .
$$

Prova. Seja $T^{*}$ uma árvore $k$-restrita mínima de $(G, c, R)$ e, para cada componente cheio $T_{j}^{*}$ de $T^{*}$, considere $\tau_{j}^{*}$ os terminais de $T_{j}^{*}$ para $1 \leq j \leq l$. Por $(5.1)$, temos que

$$
g_{p}\left(M_{i-1}, \tau_{j}^{*}\right) \leq \frac{c\left(T_{j}^{*}\right)+\frac{1}{2} \operatorname{perda}\left(T_{j}^{*}, c, \tau_{j}^{*}\right)}{\operatorname{ind}\left(M_{i-1}, \dot{c}_{i-1}, \tau_{j}^{*}\right)} .
$$

Pela escolha de $\tau_{i}$ na linha 5 do algoritmo, temos que

$$
g_{p}\left(M_{i-1}, \tau_{i}\right) \leq \min _{j}\left\{g_{p}\left(M_{i-1}, \tau_{j}^{*}\right)\right\} \leq \min _{j}\left\{\frac{c\left(T_{j}^{*}\right)+\frac{1}{2} \operatorname{perda}\left(T_{j}^{*}, c, \tau_{j}^{*}\right)}{i n d\left(M_{i-1}, \dot{c}_{i-1}, \tau_{j}^{*}\right)}\right\} .
$$

Sabemos que, para números não-negativos $a_{j}$ e $b_{j}, 1 \leq j \leq l$,

$$
\min _{j} \frac{a_{j}}{b_{j}} \leq \frac{\sum_{j} a_{j}}{\sum_{j} b_{j}}
$$

Tomando-se $a_{j}=c\left(T_{j}^{*}\right)+\frac{1}{2} \operatorname{perd} a\left(T_{j}^{*}, c, \tau_{j}^{*}\right)$ e $b_{j}=i n d\left(M_{i-1}, \dot{c}_{i-1}, \tau_{j}^{*}\right)$, por (5.3), temos que

$$
g_{p}\left(M_{i-1}, \tau_{i}\right) \leq \frac{\sum_{j}\left(c\left(T_{j}^{*}\right)+\frac{1}{2} \operatorname{perda}\left(T_{j}^{*}, c, \tau_{j}^{*}\right)\right)}{\sum_{j} \operatorname{ind}\left(M_{i-1}, \dot{c}_{i-1}, \tau_{j}^{*}\right)} .
$$

Pelo corolário 1.3, o denominador do lado direito da desigualdade acima pode ser substituído. Assim, obtemos

$$
g_{p}\left(M_{i-1}, \tau_{i}\right) \leq \frac{\sum_{j=1}^{l}\left(c\left(T_{j}^{*}\right)+\frac{1}{2} \operatorname{perda}\left(T_{j}^{*}, c, \tau_{j}^{*}\right)\right)}{\sum_{j=1}^{l} \operatorname{ind}\left(M_{j}^{\prime}, \dot{c}_{i-1}\left[\tau_{1}^{*}, \ldots, \tau_{j-1}^{*}\right], \tau_{j}^{*}\right)},
$$

onde $M_{j}^{\prime}$ é uma árvore geradora mínima de $\left(G[R], \dot{c}_{i-1}\left[\tau_{1}^{*}, \ldots, \tau_{j-1}^{*}\right]\right)$. Pelo lema 5.1 e por que $\sum_{j=1}^{l} c\left(T_{j}^{*}\right)=c\left(T^{*}\right)=\operatorname{smt}_{k}(G, c, R)$, temos que

$$
g_{p}\left(M_{i-1}, \tau_{i}\right) \leq \frac{\operatorname{smt}_{k}(G, c, R)+\frac{1}{2} \cdot \frac{1}{2} \operatorname{smt}_{k}(G, c, R)}{\sum_{j=1}^{l} \operatorname{ind}\left(M^{\prime}, \dot{c}_{i-1}\left[\tau_{1}^{*}, \ldots, \tau_{j-1}^{*}\right], \tau_{j}^{*}\right)} .
$$


Pela definição de índice, o denominador do lado direito da desigualdade acima é uma soma telescópica. Então

$$
\begin{aligned}
g_{p}\left(M_{i-1}, \tau_{i}\right) & \leq \frac{\frac{5}{4} \operatorname{smt}_{k}(G, c, R)}{m s t\left(G[R], \dot{c}_{i-1}\right)-m s t\left(G[R], \dot{c}_{i-1}\left[\tau_{1}^{*}, \ldots, \tau_{l}^{*}\right]\right)} \\
& =\frac{5}{4} \frac{s m t_{k}(G, c, R)}{m s t\left(G[R], \dot{c}_{i-1}\right)},
\end{aligned}
$$

já que $\operatorname{mst}\left(G[R], \dot{c}_{i-1}\left[\tau_{1}^{*}, \ldots, \tau_{l}^{*}\right]\right)=0$ pois $\bigcup_{j=1}^{l} T_{j}^{*}=T^{*}$ e conecta $R$.

Para simplificar as fórmulas, sejam $m_{i}:=\operatorname{mst}\left(G[R], \dot{c}_{i}\right)$, perda $a_{i}:=$ $\operatorname{perda}\left(T_{i}, c, \tau_{i}\right), \operatorname{smt}_{k}:=\operatorname{smt}_{k}(G, c, R)$ e $\operatorname{smt}:=\operatorname{smt}(G, c, R)$. Usando a definição de ganho ponderado, temos que

$$
g_{p}\left(M_{i-1}, \tau_{i}\right)=\frac{c\left(T_{i}\right)+\frac{1}{2} \operatorname{perd}_{i}}{\operatorname{ind}\left(M_{i-1}, \dot{c}_{i-1}, \tau_{i}\right)}=\frac{c\left(T_{i}\right)+\frac{1}{2} \operatorname{perd}_{i}}{m_{i-1}-m_{i}}
$$

Portanto, aplicando-se a igualdade acima e as desigualdades (5.2) e (5.4),

$$
\begin{aligned}
\sum_{i=1}^{f}\left(c\left(T_{i}\right)+\frac{1}{2} \operatorname{per} a_{i}\right) & =\sum_{i=1}^{f} g_{r}\left(M_{i-1}, \tau_{i}\right)\left(m_{i-1}-m_{i}\right) \\
& \leq \sum_{i=1}^{f} \min \left\{1, \frac{5}{4} \frac{s m t_{k}}{m_{i-1}}\right\}\left(m_{i-1}-m_{i}\right) .
\end{aligned}
$$

Note que a seqüência $m_{0}, m_{1}, \ldots, m_{f}$, que são os custos das árvores geradoras mínimas em cada iteração do algoritmo, é monotonicamente decrescente, sendo que $m_{f}=0$ e $m_{0}=$ $m s t(G[R], \dot{c})$. Assim, podemos limitar o somatório em (5.5) por uma integral do seguinte modo.

$$
\begin{aligned}
\sum_{i=1}^{f} \min \left\{1, \frac{5}{4} \frac{s m t_{k}}{m_{i-1}}\right\}\left(m_{i-1}-m_{i}\right) & \leq \sum_{i=1}^{f} \int_{m_{i}}^{m_{i-1}} \min \left\{1, \frac{5}{4} \frac{s m t_{k}}{x}\right\} d x \\
& =\int_{m_{f}}^{m_{0}} \min \left\{1, \frac{5}{4} \frac{s m t_{k}}{x}\right\} d x \\
& =\int_{0}^{\frac{5}{4} s m t_{k}} d x+\frac{5}{4} s m t_{k} \int_{\frac{5}{4} s m t_{k}}^{m_{0}} \frac{1}{x} d x \\
& =\frac{5}{4} s m t_{k}+\frac{5}{4} s m t_{k}\left(\ln \frac{m_{0}}{\frac{5}{4} s m t_{k}}\right) \\
& =\frac{5}{4} s m t_{k}\left(1+\ln \frac{4}{5} \frac{m_{0}}{s m t_{k}}\right)
\end{aligned}
$$

e com isso concluímos a prova do lema.

A seguir vamos nos dedicar à análise da segunda fase do algoritmo do pré-processamento. Primeiramente mostraremos um delimitador superior no custo de uma árvore $k$-restrita mínima de $(G, c, R \cup W)$. 
Lema 5.3 Sejam $G$ um grafo completo, $c$ uma função de $E_{G}$ em $Q_{>0}$ satisfazendo a desigualdade triangular, $R$ um conjunto de vértices de $G$ e $W$ o conjunto de vértices de $G$ construído na primeira fase do algoritmo do pré-processamento. Se $T_{1}, \ldots, T_{f}$ são as árvores $k$-restritas selecionadas pelo algoritmo do pré-processamento para $(G, c, R)$ e $\tau_{1}, \ldots, \tau_{f}$ seus respectivos conjuntos de terminais, então

$$
\operatorname{smt}_{k}(G, c, R \cup W) \leq \operatorname{smt}_{k}(G, c, R)+\sum_{i=1}^{f} \operatorname{perda}\left(T_{i}, c, \tau_{i}\right) .
$$

Prova. Seja $T^{*}$ uma árvore $k$-restrita mínima de $(G, c, R)$ e $F_{i}$ uma floresta de terminais mínima de $\left(T_{i}, c, \tau_{i}\right)$ para $1 \leq i \leq f$. Para provar o lema, basta mostrar que o grafo $T^{*}+F_{1}+\cdots+F_{f}$ contém uma árvore $k$-restrita de $(G, R \cup W)$.

Primeiro note que $T^{*}+F_{1}+\cdots+F_{f}$ é conexo, pois $\bigcup_{i=1}^{f} \tau_{i}=R$, todo componente conexo de $F_{i}$ contém pelo menos um terminal de $\tau_{i}$ e $T^{*}$ conecta todos os terminais. Assim, seja $T^{\prime}$ uma árvore geradora de $T^{*}+F_{1}+\cdots+F_{f}$. Basta mostrar que $T^{\prime}$ é uma árvore $k$-restrita de $(G, R \cup W)$.

Note que toda aresta de $F_{i}$ que está em $T^{\prime}$ é um componente cheio de $T^{\prime}$, pois todo vértice de $F_{i}$ é terminal em $T^{\prime}$. (Para todo $1 \leq i \leq f$, temos que $V S\left(T_{i}\right) \subseteq W$.) Os demais componentes cheios de $T^{\prime}$ são subárvores de componentes cheios de $T^{*}$ e portanto têm no máximo o mesmo número de folhas que o correspondente componente cheio de $T^{*}$, ou seja, são também $k$-árvores. Logo $T_{k}^{\prime}$ é uma árvore $k$-restrita de $(G, R \cup W)$ e de fato $T^{*}+F_{1}+\cdots+F_{f}$ contém uma árvore $k$-restrita de $(G, R \cup W)$. Como $\operatorname{smt}_{k}(G, c, R \cup W) \leq c\left(T^{\prime}\right) \leq \operatorname{smt}_{k}(G, c, R)+\sum_{i=1}^{f} \operatorname{perda}\left(T_{i}, c, \tau_{i}\right)$, o lema segue.

O teorema abaixo mostra uma razão de aproximação para o algoritmo do pré-processamento.

Teorema 5.4 Sejam $G$ um grafo completo, c uma função de $E_{G}$ em $Q_{>0}$ satisfazendo a desigualdade triangular e $R$ um conjunto de vértices de $G$. A razão entre o custo da solução do algoritmo do pré-processamento e o custo de uma árvore de Steiner mínima de $(G, c, R)$ é menor que 1,645 para $k$ suficientemente grande.

Prova. Considere o conjunto $W$ de vértices de Steiner construído pelo algoritmo e sejam $T_{1}, \ldots, T_{f}$ as árvores $k$-restritas mínimas selecionadas pelo algoritmo para $(G, c, R)$ e $\tau_{1}, \ldots, \tau_{f}$ seus respectivos conjuntos de terminais. Para simplicar, denotemos $\operatorname{mst}(G[R], c)$ por $m s t, s m t(G, c, R)$ por $s m t, s m t_{k}(G, c, R)$ por $s m t_{k}$ e $s m t_{k}(G, c, R \cup W)$ por $s m t_{k}^{\prime}$.

Pelo lema 4.2 do algoritmo do ganho relativo, temos que

$$
c\left(T^{\prime}\right) \leq\left(1+\ln \frac{m s t}{s m t_{k}^{\prime}}\right) s m t_{k}^{\prime} \leq\left(1+\ln \frac{\sum_{i=1}^{f} c\left(T_{i}\right)}{s m t_{k}^{\prime}}\right) s m t_{k}^{\prime},
$$

pois $m s t \leq \sum_{i=1}^{f} c\left(T_{i}\right)$ 
Seja

$$
B_{k}:=\frac{5}{4} s m t_{k}\left(1+\ln \frac{4}{5} \frac{m s t}{s m t_{k}}\right)
$$

Pelo lema 5.2, temos que

$$
\sum_{i=1}^{f} c\left(T_{i}\right) \leq B_{k}-\frac{1}{2} \sum_{i=1}^{f} \operatorname{perd} a\left(T_{i}, c, \tau_{i}\right)=\frac{1}{2}\left(2 B_{k}-\sum_{i=1}^{f} \operatorname{perd} a\left(T_{i}, c, \tau_{i}\right)\right)
$$

e, combinando o lema 5.3, concluímos que

$$
\sum_{i=1}^{f} c\left(T_{i}\right) \leq \frac{1}{2}\left(2 B_{k}+s m t_{k}-s m t_{k}^{\prime}\right)
$$

Aplicando-se (5.7) em (5.6), deduzimos que

$$
\begin{aligned}
c\left(T^{\prime}\right) & \leq\left(1+\ln \frac{2 B_{k}+s m t_{k}-s m t_{k}^{\prime}}{2 s m t_{k}^{\prime}}\right) s m t_{k}^{\prime} \\
& =f\left(\frac{a_{k}-s m t_{k}^{\prime}}{2}\right)
\end{aligned}
$$

onde $a_{k}:=2 B_{k}+s m t_{k} \mathrm{e}$

$$
f(x)=\left(1+\ln \frac{x}{a_{k}-2 x}\right)\left(a_{k}-2 x\right) .
$$

Mas o máximo da função $f(x)$ é atingido quando a sua derivada é nula, ou seja, quando

$$
\frac{a_{k}-2 x}{2 x}=\ln \frac{x}{a_{k}-2 x}
$$

Definindo $y=\frac{a_{k}-2 x}{2 x}$, a equação acima corresponde a $y=\ln \frac{1}{2 y}$, cuja solução é $y_{0}:=$ $0,3515 \ldots$.... Portanto, como a solução de (5.9) é $x_{0}:=\frac{a_{k}}{2\left(1+y_{0}\right)}$, então o máximo da função $f(x)$ é

$$
f\left(x_{0}\right)=\left(1+\ln \frac{x_{0}}{2 x_{0} y_{0}}\right) 2 x_{0} y_{0}=\left(1+y_{0}\right) 2 y_{0} \frac{a_{k}}{2\left(1+y_{0}\right)}=a_{k} y_{0}=\left(2 B_{k}+s m t_{k}\right) y_{0}
$$

pois $a_{k}-2 x_{0}=2 x_{0} y_{0}$ e $y_{0}=\ln \frac{1}{2 y_{0}}$.

Assim, de (5.8), concluímos que

$$
c\left(T^{\prime}\right) \leq f\left(x_{0}\right)=\left(2 B_{k}+s m t_{k}\right) y_{0}
$$


e o limite da razão de aproximação do algoritmo do pré-processamento quando $k$ tende a infinito é

$$
\begin{aligned}
\lim _{k \rightarrow \infty} \frac{c\left(T^{\prime}\right)}{s m t} & \leq \lim _{k \rightarrow \infty} \frac{\left(s m t_{k}+2 B_{k}\right) y_{0}}{s m t} \\
& =y_{0}\left(\lim _{k \rightarrow \infty} \frac{s m t_{k}}{s m t}+2 \lim _{k \rightarrow \infty} \frac{B_{k}}{s m t}\right) \\
& \leq y_{0}\left(1+\frac{5}{2}\left(1+\ln \frac{8}{5}\right)\right) \\
& \leq 1,6443 \ldots
\end{aligned}
$$

Portanto, para $k$ suficientemente grande, temos que

$$
\frac{c\left(T^{\prime}\right)}{s m t}<1,645
$$

A escolha de $1 / 2$ como fator multiplicativo da perda na definição do ganho ponderado resulta na melhor razão de aproximação obtida por esta análise e, como nos algoritmos anteriores, não se conhece instância do PSG que, quando executado o algoritmo do préprocessamento, resulte na razão de aproximação demonstrada.

Hougardy e Prömel [27] generalizaram o algoritmo do pré-processamento. Ao invés de duas fases, o algoritmo proposto por eles é composto por $n$ fases. Basicamente, em cada uma delas o algoritmo escolhe algumas árvores $k$-restritas e adiciona seus vértices de Steiner ao conjunto de terminais formando um novo conjunto de terminais para a fase seguinte processar. Eles mostraram que este algoritmo atinge uma razão de aproximação de no máximo 1,598 para $n$ e $k$ suficientemente grandes. 


\section{Capítulo 6}

\section{Algoritmo de Robins e Zelikovsky}

Este algoritmo, projetado por Robins e Zelikovsky [42], é o melhor algoritmo de aproximação conhecido para o PSG até o momento. Como os algoritmos apresentados nos capítulos anteriores, ele também utiliza árvores $k$-restritas, onde $k$ é uma constante fixa maior ou igual a 3, para produzir como solução uma árvore de Steiner.

A novidade deste algoritmo em relação aos anteriores está na utilização do ganho relativo à perda e da contração de florestas de terminais. A contração de florestas de terminais de $\left(T_{\tau}, \tau\right)$ em contraposição à contração de $T_{\tau}$ possibilita a escolha, em uma iteração futura, de uma árvore $k$-restrita que contenha mais de um terminal de uma árvore $k$-restrita já escolhida. Com exceção do algoritmo de Berman e Ramayer, essa possibilidade não existe nos algoritmos anteriores.

\subsection{Contração de florestas de terminais e ganho re- lativo à perda}

Sejam $G$ um grafo completo, $c$ uma função de $E_{G}$ em $Q_{>0}$ satisfazendo a desigualdade triangular e $R$ um conjunto de vértices - os terminais. Considere também uma função $\dot{c}$ de $E_{G[R]}$ em $Q_{>0}$, um conjunto de pelo menos dois terminais $\tau$, uma árvore de Steiner $T_{\tau}$ de $(G, \tau)$ e uma floresta de terminais mínima $F$ de $\left(T_{\tau}, c, \tau\right)$.

Para cada aresta $e$ de $T_{\tau}-F$ seja $q(e)$ a aresta de $G[R]$ cujos extremos são os terminais de cada um dos componentes de $F$ conectados pela aresta $e$. Se $|\tau|=2$ então $e=q(e)$. A contração de $F$ em $\dot{c}$ é a função que denotamos por $\dot{c}\left[T_{\tau}, F\right]$ de $E_{G[R]}$ em $Q_{>0}$, definida. por

$$
\dot{c}\left[T_{\tau}, F\right](f)= \begin{cases}\dot{c}(f) & \text { se } f \neq q(e) \text { para todo } e \text { em } E_{T_{\tau}-F} \\ \min \{\dot{c}(f), c(e)\} & \text { se } f=q(e) \text { para algum } e \text { em } E_{T_{\tau}-F} .\end{cases}
$$

Para simplificar, denotamos $\dot{c}\left[T_{1}, F_{1}\right]\left[T_{2}, F_{2}\right] \cdots\left[T_{i}, F_{i}\right]$ por $\dot{c}\left[T_{1} \ldots T_{i}, F_{1} \ldots F_{i}\right]$. 
A função $q$ definida nas arestas de $T_{\tau}-F$ é denominada de projeção da contração de $F$ em $T_{\tau}$ relativa a $G[R]$. Denotamos por $Q\left(T_{\tau}, F\right)$ a imagem da função $q$. Veja na figura 6.1 um exemplo de contração de florestas de terminais. Seja $T$ o componente cheio da figura. As linhas grossas representam as arestas de uma floresta de terminais mínima $F$ de $T$. Para cada aresta $e$ (linha normal) de $T-F$, o custo da aresta $q(e)$ (linha pontilhada) é alterado para o $\min \{c(e), \dot{c}(q(e))\}$.

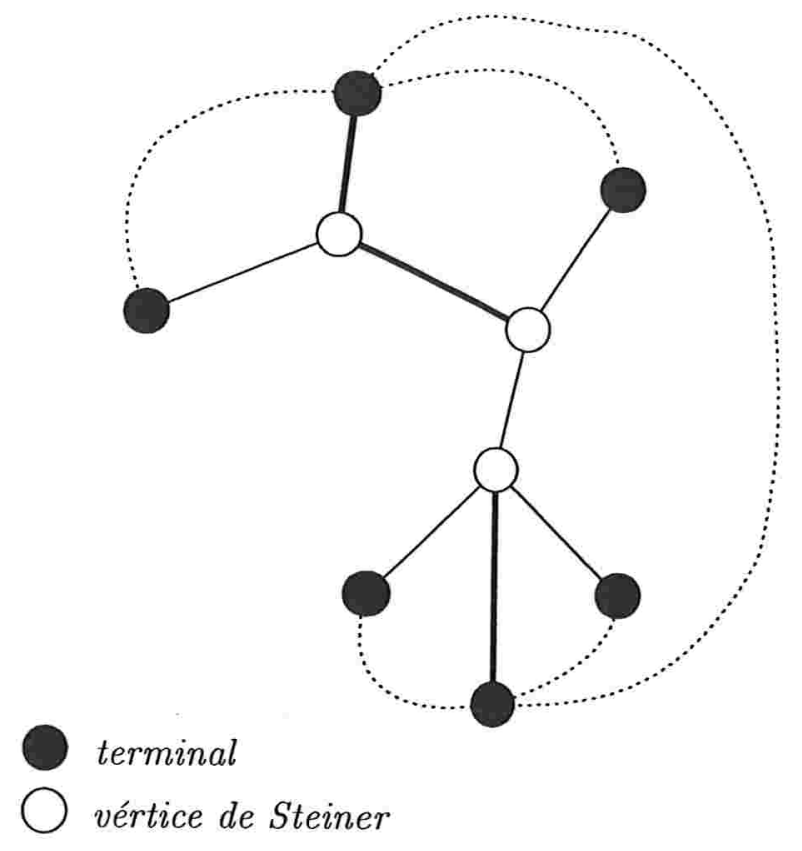

Figura 6.1: Exemplo de contração de uma floresta de terminais.

Nosso objetivo, através do lema a seguir, é mostrar uma desigualdade envolvendo floresta de terminais e contração de florestas de terminais muito semelhante a uma propriedade de conjunto remoção provada no lema 1.2.

Lema 6.1 Sejam $G$ um grafo, c uma função de $E_{G}$ em $Q_{>0}, R$ um conjunto de vértices de $G, \dot{c}$ a restrição de c a $G[R], \tau_{1}$ um conjunto de vértices de $R, T_{1}$ uma árvore de Steiner de $\left(G, \tau_{1}\right)$ e $F_{1}$ uma floresta de terminais mínima de $\left(T_{1}, c, \tau_{1}\right)$. Se $\dot{c}^{\prime}$ é uma função em $E_{G[R]}$ que coincide com $\dot{c}$ exceto em uma aresta e de $G[R]$ onde $\dot{c}^{\prime}(e) \leq \dot{c}(e)$, então

$$
\operatorname{mst}(G[R], \dot{c})-\operatorname{mst}\left(G[R], \dot{c}\left[T_{1}, F_{1}\right]\right) \geq \operatorname{mst}\left(G[R], \dot{c}^{\prime}\right)-\operatorname{mst}\left(G[R], \dot{c}^{\prime}\left[T_{1}, F_{1}\right]\right) .
$$

Prova. Sejam $e_{1}, \ldots, e_{j}$ as arestas de $T_{1}-F_{1}$ e $q$ a projeção da contração de $F_{1}$ em $T_{1}$ relativa a $G[R]$. Seja $M_{0}$ uma árvore geradora mínima de $(G[R], \dot{c})$ e $\dot{c}_{0}=\dot{c}$. Para 
$i=1, \ldots, j$, seja $\dot{c}_{i}$ a função custo definida da seguinte maneira

$$
\dot{c}_{i}(f)= \begin{cases}\dot{c}_{i-1}(f) & \text { se } f \neq q\left(e_{i}\right), \\ \min \left\{\dot{c}_{i-1}(f), c\left(e_{i}\right)\right\} & \text { se } f=q\left(e_{i}\right)\end{cases}
$$

e seja $M_{i}$ uma árvore geradora mínima de $\left(G[R], \dot{c}_{i}\right)$. Note que

$$
\dot{c}_{i-1}\left(M_{i-1}\right)-\dot{c}_{i}\left(M_{i}\right)=\max \left\{0, \operatorname{ind}\left(M_{i-1}, \dot{c}_{i-1}, q\left(e_{i}\right)\right)-c\left(e_{i}\right)\right\} .
$$

Portanto, como $\dot{c}_{j}=\dot{c}\left[T_{1}, F_{1}\right]$, temos que

$$
\operatorname{mst}(G[R], \dot{c})-\operatorname{mst}\left(G[R], \dot{c}\left[T_{1}, F_{1}\right]\right)=\sum_{i=1}^{j} \max \left\{0, \operatorname{ind}\left(M_{i-1}, \dot{c}_{i-1}, q\left(e_{i}\right)\right)-c\left(e_{i}\right)\right\} \text {. }
$$

Agora, seja $\dot{c}_{0}^{\prime}=\dot{c}^{\prime}$ e $\dot{c}_{i}^{\prime}$ uma função custo definida do seguinte modo

$$
\dot{c}_{i}^{\prime}(f)= \begin{cases}\dot{c}_{i}(f) & \text { se } f \neq e \\ \min \left\{\dot{c}_{i}(f), \dot{c}^{\prime}(e)\right\} & \text { se } f=e\end{cases}
$$

Seja

$$
M_{i}^{\prime}:= \begin{cases}M_{i} & \text { se } \dot{c}_{i}^{\prime}(e) \geq \operatorname{ind}\left(M_{i}, \dot{c}_{i}, e\right), \\ M_{i}-e^{\prime}+e & \text { se } \dot{c}_{i}^{\prime}(e)<i n d\left(M_{i}, \dot{c}_{i}, e\right) \text { e } e^{\prime} \text { é o índice de e em } M_{i} .\end{cases}
$$

Note que $M_{i}^{\prime}$ é uma árvore geradora mínima de $\left(G[R], \dot{c}_{i}^{\prime}\right)$. Para $i=1, \ldots, j$ temos, pelo lema 1.2 , que

$$
\operatorname{ind}\left(M_{i-1}, \dot{c}_{i-1}, e_{i}\right) \geq \operatorname{ind}\left(M_{i-1}^{\prime}, \dot{c}_{i-1}^{\prime}, e_{i}\right)
$$

Além disso,

$$
\dot{c}_{i-1}^{\prime}\left(M_{i-1}^{\prime}\right)-\dot{c}_{i}^{\prime}\left(M_{i}^{\prime}\right)=\max \left\{0, \operatorname{ind}\left(M_{i-1}^{\prime}, \dot{c}_{i-1}^{\prime}, q\left(e_{i}\right)\right)-c\left(e_{i}\right)\right\} .
$$

Portanto, como $\dot{c}_{j}^{\prime}=\dot{c}^{\prime}\left[T_{1}, F_{1}\right]$, temos que

$$
\operatorname{mst}\left(G[R], \dot{c}^{\prime}\right)-\operatorname{mst}\left(G[R], \dot{c}^{\prime}\left[T_{1}, F_{1}\right]\right)=\sum_{i=1}^{j} \max \left\{0, \operatorname{ind}\left(M_{i-1}^{\prime}, \dot{c}_{i-1}^{\prime}, q\left(e_{i}\right)\right)-c\left(e_{i}\right)\right\} \text {. }
$$

Por (6.1), (6.2) e (6.3) podemos concluir que

$$
\operatorname{mst}(G[R], \dot{c})-\operatorname{mst}\left(G[R], \dot{c}\left[T_{1}, F_{1}\right]\right) \geq \operatorname{mst}\left(G[R], \dot{c}^{\prime}\right)-\operatorname{mst}\left(G[R], \dot{c}^{\prime}\left[T_{1}, F_{1}\right]\right) \text {. }
$$


Corolário 6.2 Sejam $G$ um grafo, c uma função de $E_{G}$ em $Q_{>0}, R$ um conjunto de vértices de $G$ e $\dot{c}$ a restrição de c a $G[R]$. Respectivamente, sejam $\tau_{0}$ e $\tau_{1}$ conjuntos nãovazios de vértices de $R, T_{0}$ e $T_{1}$ árvores de Steiner de $\left(G, \tau_{0}\right)$ e $\left(G, \tau_{1}\right), F_{0}$ e $F_{1}$ florestas de terminais mínima de $\left(T_{0}, c, \tau_{0}\right)$ e $\left(T_{1}, c, \tau_{1}\right)$. Então

$$
\operatorname{mst}(G[R], \dot{c})-\operatorname{mst}\left(G[R], \dot{c}\left[T_{1}, F_{1}\right]\right) \geq \operatorname{mst}\left(G[R], \dot{c}\left[T_{0}, F_{0}\right]\right)-\operatorname{mst}\left(G[R], \dot{c}\left[T_{0}, F_{0}\right]\left[T_{1}, F_{1}\right]\right) .
$$

Prova. Basta aplicar sucessivamente o lema 6.1 com cada aresta de $Q\left[T_{0}, F_{0}\right]$ no papel de $e$.

O ganho relativo à perda de $\tau$ em $\dot{c}$, denotado por $g_{l}(\tau, \dot{c})$, é dado por

$$
g_{l}(\tau, \dot{c}):=\min _{T_{\tau}, F}\left\{\frac{\operatorname{perda}\left(T_{\tau}, c, \tau\right)}{\operatorname{mst}(G[R], \dot{c})-\operatorname{mst}\left(G[R], \dot{c}\left[T_{\tau}, F\right]\right)}\right\},
$$

onde o mínimo é tomado dentre todas as árvores $k$-restritas $T_{\tau}$ de $(G, \tau)$ e todas as florestas de terminais mínimas $F$ de $\left(T_{\tau}, c, \tau\right)$. Se o denominador for nulo, considere $g_{l}(\tau, \dot{c}):=+\infty$.

Note que o denominador do ganho relativo à perda é uma variante do custo de um conjunto remoção, que utiliza a contração de florestas de terminais no lugar da função redução.

\subsection{Descrição do algoritmo}

O algoritmo depende de um parâmetro fixo $k$. A entrada do algoritmo é um grafo completo $G$, uma função $c$ de $E_{G}$ em $Q_{>0}$ satisfazendo a desigualdade triangular e um conjunto $R$ de vértices de $G$. Como saída, o algoritmo produz uma árvore de Steiner de $(G, R)$ cujo custo dividido pelo custo de uma solução ótima do PSG para $(G, c, R)$, para $k$ suficientemente grande, é no máximo 1,55.

O algoritmo começa com uma função $\dot{c}$ que inicialmente é a restrição de $c$ a $G[R]$ e um conjunto $W$ de vértices inicialmente vazio. Em cada iteração, o algoritmo escolhe $\tau \subseteq R$ com $3 \leq|\tau| \leq k$, uma árvore $k$-restrita $T_{\tau}$ de $(G, \tau)$ e uma floresta de terminais $F$ de $\left(T_{\tau}, \tau\right)$ que minimiza $g_{l}(\tau, \dot{c})$. O algoritmo armazena os vértices de Steiner de $T_{\tau}$ em $W$ e começa uma nova iteração com a contração de $F$ em $\dot{c}$ no lugar de $\dot{c}$.

O algoritmo pára quando não existe $\tau$ tal que $3 \leq|\tau| \leq k$ e $g_{l}(\tau, \dot{c})<1$. Neste ponto, o algoritmo constrói uma árvore geradora mínima de $(G[R \cup W], c)$ como saída do algoritmo. Na figura 6.2, está a descrição precisa do algoritmo de Robins e Zelikovsky.

Note que o algoritmo de Robins e Zelikovsky pode ser implementado de forma que seu consumo de tempo seja polinomial.

É importante reforçar que em uma iteração, ao escolhermos uma árvore $k$-restrita, existe a possibilidade de que pares de terminais desta árvore $k$-restrita possam ser compartilhados por uma outra árvore $k$-restrita escolhida futuramente pelo algoritmo. Assim, por causa da contração de florestas de terminais, o algoritmo está descartando menos árvores k-restritas do que se fosse utilizada a operação de redução. 
Algoritmo de Robins e Zelikovsky $(G, c, R)$

$1 \quad$ Seja $\dot{c}_{0}$ a restrição de $c$ a $G[R]$

$2 \quad W_{0}:=\emptyset$

$3 \quad i:=1$;

4 Enquanto existir $\tau \subseteq R$ com $3 \leq|\tau| \leq k$ tal que $g_{l}\left(\tau, \dot{c}_{i-1}\right)<1$ faça

$5 \quad$ Seja $\tau_{i} \subseteq R$ com $3 \leq\left|\tau_{i}\right| \leq k$ e $g_{l}\left(\tau_{i}, \dot{c}_{i-1}\right)$ mínimo;

$6 \quad$ Sejam $T_{i}$ uma árvore $k$-restrita de $\left(G, c, \tau_{i}\right)$

e $F_{i}$ uma floresta de terminais mínima de $\left(T_{i}, c, \tau_{i}\right)$ para as quais

$g_{l}\left(\tau_{i}, \dot{c}_{i-1}\right)=\frac{p e r d a\left(T_{i}, c_{i-1}, \tau_{i}\right)}{m s t\left(G[R], \dot{c}_{i-1}\right)-m s t\left(G[R], \dot{c}_{i-1}\left[F_{i}, \tau_{i}\right]\right)}$

$7 \quad W_{i}:=W_{i-1} \cup V S\left(T_{i}\right)$;

$8 \quad \dot{c}_{i}:=\dot{c}_{i-1}\left[T_{i}, F_{i}\right]$

$9 \quad i:=i+1$

$10 f:=i-1$;

11 Seja $T$ uma árvore geradora mínima de $\left(G\left[R \cup W_{f}\right], c\right)$;

12 Devolva $T$.

Figura 6.2: Algoritmo de Robins e Zelikovsky

\subsection{Análise do algoritmo de Robins e Zelikovsky}

O lema abaixo mostra um limitante inferior para o custo de uma árvore $k$-restrita mínima que será muito útil na demonstração de uma razão de aproximação do algoritmo.

Lema 6.3 Sejam $G$ um grafo completo, c uma função de $E_{G}$ em $Q_{>0}$ satisfazendo a desigualdade triangular e $R$ um conjunto de vértices de $G$. Seja $T^{*}$ uma árvore k-restrita mínima arbitrária de $(G, c, R)$ e $t$ o número de componentes cheios de $T^{*}$. Para $j=$ $1, \ldots, t$ considere as triplas $\left(T_{j}^{*}, \tau_{j}^{*}, F_{j}^{*}\right)$ onde $T_{j}^{*}$ é um componente cheio de $T^{*}, \tau_{j}^{*}$ é o conjunto dos terminais de $T_{j}^{*}$ e $F_{j}^{*}$ é uma floresta de terminais minima de $\left(T_{j}^{*}, c, \tau_{j}^{*}\right)$. Então

$$
\operatorname{smt}_{k}(G, c, R)=c\left(T^{*}\right) \geq \operatorname{mst}\left(G[R], \dot{c}\left[T_{1}^{*} \ldots T_{t}^{*}, F_{1}^{*} \ldots F_{t}^{*}\right]\right)+\operatorname{perda}\left(T^{*}, c, R\right) .
$$

Prova. Seja $Q\left(T_{j}^{*}, F_{j}^{*}\right)$ a imagem da função projeção da contração de $T_{j}^{*}$ em $F_{j}^{*}$ relativa a $G[R]$. Note que este conjunto é um conjunto minimal de arestas que conecta $\tau_{j}^{*}$, ou seja, $Q\left(T_{j}^{*}, F_{j}^{*}\right)$ é uma árvore geradora de $G\left[\tau_{j}^{*}\right]$. Como $T_{j}^{*}$ são os componentes cheios de $T^{*}$, podemos concluir que $\bigcup_{j=1}^{t} Q\left(T_{j}^{*}, F_{j}^{*}\right)$ são as arestas de uma árvore geradora $M$ de $G[R]$ pois $\bigcup_{j=1}^{t} T_{j}^{*}=T^{*}$. De fato, um circuito em $M$ induziria um circuito em $T$.

Denotando $\dot{c}\left[T_{1}^{*} \ldots T_{t}^{*}, F_{1}^{*} \ldots F_{t}^{*}\right]$ por $\dot{c}_{*}$, vale que

$$
\begin{aligned}
\operatorname{smt}_{k}(G, c, R) & =c\left(T^{*}\right) \\
& =\sum_{j=1}^{t} c\left(T_{j}^{*}\right)
\end{aligned}
$$




$$
\begin{aligned}
& =\sum_{j=1}^{t}\left(c\left(T_{j}^{*}-F_{j}^{*}\right)+c\left(F_{j}^{*}\right)\right) \\
& =\sum_{j=1}^{t} \dot{c}_{*}\left(Q\left(T_{j}^{*}, F_{j}^{*}\right)\right)+\sum_{j=1}^{t} \operatorname{perda}\left(T_{j}^{*}, c, \tau_{j}^{*}\right) \\
& =\dot{c}_{*}(M)+\operatorname{perda}\left(T^{*}, c, R\right) \\
& \geq \operatorname{mst}\left(G[R], \dot{c}_{*}\right)+\operatorname{perda}\left(T^{*}, c, R\right),
\end{aligned}
$$

concluindo a prova do lema.

Baseando-se no lema 6.3 podemos definir um delimitador superior para o custo de uma solução produzida pelo algoritmo.

Lema 6.4 Sejam $G$ um grafo completo, c uma função de $E_{G}$ em $Q_{>0}$ satisfazendo a desigualdade triangular e $R$ um conjunto de vértices de $G$. No algoritmo de Robins e Zelikovsky, temos que

$$
\operatorname{mst}\left(G\left[R \cup W_{f}\right], c\right) \leq \operatorname{mst}\left(G[R], \dot{c}_{f}\right)+\sum_{j=1}^{f} \operatorname{perda}\left(T_{j}, c, \tau_{j}\right)
$$

Prova. Seja $M$ uma árvore geradora mínima de $G\left([R], \dot{c}_{f}\right)$. Considere o subgrafo $H$ de $G\left[R \cup W_{f}\right]$ obtido de $M$ pela substituição de cada aresta $e$ em $M \cap \bigcup_{j=1}^{f} Q\left(T_{j}, F_{j}\right)$ por uma aresta $f$ em $T_{j}-F_{j}$ tal que $\dot{c}_{f}(e)=c(f)$ e pela floresta de terminais $F_{j}$ para algum $j$. Assim,

$$
c(H) \leq \dot{c}_{f}(M)+\sum_{j=1}^{f} c\left(F_{j}\right)
$$

Além disso, $H$ é um subgrafo gerador conexo de $G\left[R \cup W_{f}\right]$. De fato, para cada substituição, $F_{j}+f$ conecta os extremos da aresta $e$ removida. Portanto

$$
\operatorname{mst}\left(G\left[R \cup W_{f}\right], c\right) \leq c(H) \leq \operatorname{mst}\left(G[R], \dot{c}_{f}\right)+\sum_{j=1}^{f} c\left(F_{j}\right) .
$$

Lema 6.5 Sejam $G$ um grafo completo, c uma função de $E_{G}$ em $Q_{>0}$ satisfazendo a desigualdade triangular e $R$ um conjunto de vértices de $G$. Sejam $\dot{c}$ a restrição de $c$ a $G[R], T^{*}$ uma árvore k-restrita mínima arbitrária de $(G, c, R)$ e $t$ o número de componentes cheios de $T^{*}$. Para $j=1, \ldots, t$, considere as triplas $\left(T_{j}^{*}, \tau_{j}^{*}, F_{j}^{*}\right)$ onde $T_{j}^{*}$ é um componente cheio de $T^{*}, \tau_{j}^{*}$ é o conjunto dos terminais de $T_{j}^{*}$ e $F_{j}^{*}$ é uma floresta de terminais mínimas de $\left(T_{j}^{*}, c, \tau_{j}^{*}\right)$. No algoritmo de Robins e Zelikovsky temos que

$$
m s t\left(G[R], \dot{c}_{f}\right) \leq \operatorname{mst}\left(G[R], \dot{c}\left[T_{1}^{*} \ldots T_{t}^{*}, F_{1}^{*} \ldots F_{t}^{*}\right]\right)+\operatorname{perda}\left(T^{*}, c, R\right) .
$$


Prova. Denote $\dot{c}_{f}\left[T_{1}^{*} \ldots T_{j}^{*}, F_{1}^{*} \ldots F_{j}^{*}\right]$ por $c_{f}^{j}$ e $\dot{c}\left[T_{1}^{*} \ldots T_{j}^{*}, F_{1}^{*} \ldots F_{j}^{*}\right]$ por $c_{0}^{j}$. Pela definição de contração de florestas de terminais mínimas, temos que

$$
\operatorname{mst}\left(G[R], c_{0}^{t}\right) \geq \operatorname{mst}\left(G[R], c_{f}^{t}\right) \text {. }
$$

Pela desigualdade acima e pelo corolario 6.2, vale que

$$
\begin{aligned}
\operatorname{mst}\left(G[R], \dot{c}_{f}\right)-\operatorname{mst}\left(G[R], c^{t}\right) & \leq \operatorname{mst}\left(G[R], c_{f}^{0}\right)-\operatorname{mst}\left(G[R], c_{f}^{t}\right) \\
& =\sum_{j=1}^{t}\left(\operatorname{mst}\left(G[R], c_{f}^{j-1}\right)-\operatorname{mst}\left(G[R], c_{f}^{j}\right)\right) \\
& \leq \sum_{j=1}^{t}\left(\operatorname{mst}\left(G[R], \dot{c}_{f}\right)-\operatorname{mst}\left(G[R], \dot{c}_{f}\left[T_{j}^{*}, F_{j}^{*}\right]\right)\right) .
\end{aligned}
$$

A condição da linha 4 do algoritmo de Robins e Zelikovsky garante que $g_{l}\left(\tau_{j}^{*}, \dot{c}_{f}\right) \geq 1$, ou seja, que

$$
\operatorname{mst}\left(G[R], \dot{c}_{f}\right)-\operatorname{mst}\left(G[R], \dot{c}_{f}\left[T_{j}^{*}, F_{j}^{*}\right]\right) \leq \operatorname{perda}\left(T_{j}^{*}, c, \tau_{j}^{*}\right) .
$$

Combinando a desigualdade acima com (6.4) obtemos que

$$
\operatorname{mst}\left(G[R], \dot{c}_{f}\right)-\operatorname{mst}\left(G[R], c_{0}^{t}\right) \leq \sum_{j=1}^{t} \operatorname{perda}\left(T_{j}^{*}, c, \tau_{j}^{*}\right)=\operatorname{perda}\left(T^{*}, c, R\right)
$$

Finalmente, o próximo lema dá uma delimitação superior para o custo da solução produzida pelo algoritmo. Sua prova se assemelha muito à prova do lema 5.2.

Lema 6.6 Sejam $G$ um grafo completo, c uma função de $E_{G}$ em $Q_{>0}$ satisfazendo a desigualdade triangular e $R$ um conjunto de vértices de $G$. Seja $T^{*}$ uma árvore k-restrita minima arbitrária de $(G, c, R)$ e l o número de componentes cheios de $T^{*}$. O custo da solução produzida pelo algoritmo de Robins e Zelikovsky é delimitado por

$m s t\left(G\left[R \cup W_{f}\right], c\right) \leq\left\{\begin{array}{l}\operatorname{smt}_{k}(G, c, R) \quad \operatorname{se} \operatorname{perda}\left(T^{*}, c, R\right)=0, \\ \operatorname{smt} t_{k}(G, c, R)+\operatorname{perda}\left(T^{*}, c, R\right) \cdot \ln \left(1+\frac{m s t(G[R], c)-s m t_{k}(G, c, R)}{\operatorname{perda}\left(T^{*}, c, R\right)}\right) \\ \quad \operatorname{se} \operatorname{perda}\left(T^{*}, c, R\right)>0 .\end{array}\right.$

Prova. Considere a tripla $\left(T_{j}^{*}, \tau_{j}^{*}, F_{j}^{*}\right)$, para $j=1, \ldots, t$, tal que $T_{j}^{*}$ é um componente cheio de $T^{*}, \tau_{j}^{*}$ é o conjunto dos terminais de $T_{j}^{*}$ e $F_{j}^{*}$ é uma floresta de terminais mínima de $\left(T_{j}^{*}, c, \tau_{j}^{*}\right)$.

Se perda $\left(T^{*}, c, R\right)=0$, então $T^{*}$ é uma árvore 2-restrita mínima de $(G, c, R)$, ou seja, $T^{*}$ é uma árvore geradora mínima de $(G[R], c)$ que, nesse caso, coincide com uma. árvore de Steiner mínima de $(G, c, R)$, isto é, o algoritmo devolve uma solução ótima. 
Supondo que perda $\left(T^{*}, c, R\right)>0$, em cada iteração o algoritmo escolhe um conjunto $\tau_{i}$ de terminais que minimiza o ganho relativo à perda. Assim,

$$
g_{l}\left(\tau_{i}, \dot{c}_{i-1}\right) \leq \min _{j}\left\{g_{l}\left(\tau_{j}^{*}, \dot{c}_{i-1}\right)\right\} .
$$

Sabemos que, para números não-negativos $a_{j}$ e $b_{j}, 1 \leq j \leq t$,

$$
\min _{j} \frac{a_{j}}{b_{j}} \leq \frac{\sum_{j} a_{j}}{\sum_{j} b_{j}}
$$

Tomando $a_{j}=\operatorname{perda}\left(T_{j}^{*}, c, \tau_{j}^{*}\right)$ e $b_{j}=\operatorname{mst}\left(G[R], \dot{c}_{i-1}\right)-\operatorname{mst}\left(G[R], \dot{c}_{i-1}\left[T_{j}^{*}, F_{j}^{*}\right]\right)$ e, utilizando a definição do ganho relativo à perda, temos que

$$
\begin{aligned}
g_{l}\left(\tau_{i}, \dot{c}_{i-1}\right) & \leq \min _{j}\left\{\frac{\operatorname{perda}\left(T_{j}^{*}, c, \tau_{j}^{*}\right)}{\operatorname{mst}\left(G[R], \dot{c}_{i-1}\right)-\operatorname{mst}\left(G[R], \dot{c}_{i-1}\left[T_{j}^{*}, F_{j}^{*}\right]\right)}\right\} \\
& \leq \frac{\sum_{j} \operatorname{perda}\left(T_{j}^{*}, c, \tau_{j}^{*}\right)}{\sum_{j}\left(\operatorname{mst}\left(G[R], \dot{c}_{i-1}\right)-\operatorname{mst}\left(G[R], \dot{c}_{i-1}\left[T_{j}^{*}, F_{j}^{*}\right]\right)\right)} .
\end{aligned}
$$

Pelo corolário 6.2, o denominador acima pode ser substituído por

$$
\sum_{j=1}^{t}\left(m s t\left(G[R], \dot{c}_{i-1}\left[T_{1}^{*} \ldots T_{j-1}^{*}, F_{1}^{*} \ldots F_{j-1}^{*}\right]\right)-\operatorname{mst}\left(G[R], \dot{c}_{i-1}\left[T_{1}^{*} \ldots T_{j}^{*}, F_{1}^{*} \ldots F_{j}^{*}\right]\right)\right)
$$

que é uma soma telescópica. Assim obtemos que

$$
\begin{aligned}
g_{l}\left(\tau_{i}, \dot{c}_{i-1}\right) & \leq \frac{\operatorname{perda}\left(T^{*}, c, R\right)}{m s t\left(G[R], \dot{c}_{i-1}\right)-\operatorname{mst}\left(G[R], \dot{c}_{i-1}\left[T_{1}^{*} \ldots T_{t}^{*}, F_{1}^{*} \ldots F_{t}^{*}\right]\right)} \\
& \leq \frac{\operatorname{perda}\left(T^{*}, c, R\right)}{m s t\left(G[R], \dot{c}_{i-1}\right)-\operatorname{mst}\left(G[R], \dot{c}\left[T_{1}^{*} \ldots T_{t}^{*}, F_{1}^{*} \ldots F_{t}^{*}\right]\right)}
\end{aligned}
$$

já que, pela definição de contração de florestas de terminais, é fácil ver que $\operatorname{mst}\left(G[R], \dot{c}_{i-1}\left[T_{1}^{*} \ldots T_{t}^{*}, F_{1}^{*} \ldots F_{t}^{*}\right]\right) \leq \operatorname{mst}\left(G[R], \dot{c}\left[T_{1}^{*} \ldots T_{t}^{*}, F_{1}^{*} \ldots F_{t}^{*}\right]\right)$.

Para simplificar as fórmulas, sejam $m^{*}:=\operatorname{mst}\left(G[R], \dot{c}\left[T_{1}^{*} \ldots T_{t}^{*}, F_{1}^{*} \ldots F_{t}^{*}\right]\right)$, perda $a_{i}:=$ $\operatorname{perda}\left(T_{i}, c, \tau_{i}\right), \operatorname{perda} a^{*}:=\operatorname{perda}\left(T^{*}, c, R\right), m_{i}:=\operatorname{mst}\left(G[R], \dot{c}_{i}\right)$ e $\operatorname{smt}_{k}:=\operatorname{smt}_{k}(G, c, R)$. Pela escolha de $\tau_{i}, T_{i}$ e $F_{i}$, temos que

$$
\sum_{i=1}^{f} \operatorname{perd}_{i}=\sum_{i=1}^{f} g_{l}\left(\tau_{i}, \dot{c}_{i-1}\right)\left(m_{i-1}-m_{i}\right)
$$

Substituindo na expressão acima $g_{l}\left(\tau_{i}, \dot{c}_{i-1}\right)$ pela melhor delimitação entre as dadas por (6.6) e pela condição de parada do algoritmo, temos que

$$
\sum_{i=1}^{f} \operatorname{perd} a_{i} \leq \sum_{i=1}^{f} \min \left\{1, \frac{\operatorname{perd} a^{*}}{m_{i-1}-m^{*}}\right\}\left(m_{i-1}-m_{i}\right) .
$$


Note que $m_{0}=\operatorname{mst}\left(G[R], \dot{c}_{0}\right)$ e $m_{f}=\operatorname{mst}\left(G[R], \dot{c}_{f}\right)$. Assim,

$$
\begin{aligned}
\sum_{i=1}^{f} \operatorname{perda}_{i} & \leq \int_{m_{f}-m^{*}}^{m_{0}-m^{*}} \min \left\{1, \frac{\operatorname{perd} a^{*}}{x}\right\} d x \\
& \leq \int_{m_{f}-m^{*}}^{\text {perda* }} d x+\text { perd } d a^{*} \int_{\text {perda }}^{m_{0}-m^{*}} \frac{d x}{x} \\
& =\operatorname{perda}^{*}+m^{*}-m_{f}+\text { perda } a^{*} \cdot \ln \left(\frac{m_{0}-m^{*}}{\operatorname{perda}^{*}}\right) \\
& \leq s m t_{k}-m_{f}+\text { perda } a^{*} \cdot \ln \left(1+\frac{m_{0}-s m t_{k}}{\operatorname{perda}^{*}}\right) .
\end{aligned}
$$

Primeiramente observe em (6.7) que $m_{0}-m^{*} \geq$ perda $a^{*} \geq m_{f}-m^{*}$. De fato, $m_{0} \geq s m t_{k} \geq$ perd $d a^{*}+m^{*}$ pelo lema 6.3 e que $m_{f} \leq m^{*}+$ perd $a^{*}$ pelo lema 6.5 . Portanto a quebra da integral é válida. A desigualdade (6.8) vale pois a derivada da função

$$
f(x)=x+\operatorname{perd} a^{*} \cdot \ln \left(\frac{m_{0}-x}{p e r d a^{*}}\right)
$$

é não-negativa para $x \leq s m t_{k}-$ perda* e portanto $f\left(m^{*}\right) \leq f\left(s m t_{k}-\right.$ perda* $)$ já que $m^{*} \leq s m t_{k}-$ perda* pelo lema 6.3.

Pelo lema 6.4 sabemos que $\operatorname{mst}\left(G\left[R \cup W_{f}\right], c\right) \leq m_{f}+\sum_{i=1}^{f} p e r d a_{i}$. Logo

$$
m s t\left(G\left[R \cup W_{f}\right], c\right) \leq s m t_{k}+\operatorname{perda}^{*} \cdot \ln \left(1+\frac{m_{0}-s m t_{k}}{p e r d a^{*}}\right),
$$

e com isso concluímos a prova do lema.

O teorema abaixo mostra uma razão de aproximação para o algoritmo de Robins e Zelikovsky.

Teorema 6.7 Sejam $G$ um grafo completo, c uma função de $E_{G}$ em $Q_{>0}$ satisfazendo a desigualdade triangular e $R$ um conjunto de vértices de $G$. A razão entre o custo da solução produzida pelo algoritmo de Robins e Zelikovsky e o custo de uma árvore de Steiner mínima de $(G, c, R)$ é, no máximo,

$$
r_{k}\left(1+\frac{\ln 3}{2}\right)
$$

Prova. Para simplificar, denotemos $\operatorname{mst}(G[R], c)$ por $\operatorname{mst}, \operatorname{smt}(G, c, R)$ por $s m t, s m t_{k}(G, c, R)$ por $s m t_{k}$ e $\operatorname{perda}\left(T^{*}, c, R\right)$ por perda*, onde $T^{*}$ é uma árvore $k$-restrita mínima arbitrária de $(G, c, R)$. Se perd $a^{*}=0$, o teorema vale trivialmente pelo lema 6.6. Podemos supor que perda* $>0$. Lembre-se que $m s t \leq 2 s m t \leq 2 s m t_{k}$. Se $T$ 
é uma árvore de Steiner de $(G, R)$ devolvida pelo algoritmo de Robins e Zelikovsky, o lema 6.6 e a desigualdade anterior garante que

$$
c(T) \leq s m t_{k}\left(1+\frac{p e r d a^{*}}{s m t_{k}} \cdot \ln \left(1+\frac{s m t_{k}}{p e r d a^{*}}\right)\right) .
$$

Pelo lema 5.1 temos que perda $\leq \frac{1}{2}$ smt $_{k}$. Como perda* $>0$ e $s m t_{k}>0$ podemos concluir que $0<\frac{\text { perda }^{*}}{s m t_{k}} \leq \frac{1}{2}$. A função $f(x)=x \cdot \ln \left(1+\frac{1}{x}\right)$ tem um máximo no intervalo $[0,1 / 2]$ quando $x=\frac{1}{2}$. Portanto o máximo de (6.9) é alcançado quando $\frac{p e r d a^{*}}{s m t_{k}}=\frac{1}{2}$. Assim

$$
\frac{c(T)}{s m t} \leq \frac{s m t_{k}}{s m t}\left(1+\frac{1}{2} \cdot \ln 3\right)=r_{k}\left(1+\frac{\ln 3}{2}\right),
$$

que conclui a demonstração do teorema.

Para $k$ suficientemente grande, a delimitação acima se aproxima de $1+\frac{\ln 3}{2}<1,55$.

Gröpl, Hougardy, Nierhoff e Prömel [22] mostraram uma instância onde a razão de aproximação do algoritmo de Robins e Zelikovsky quando aplicado a essa instância é de 1,2 para $k$ suficientemente grande. Não se conhece outra instância do PSG que resulte em uma razão de aproximação maior quando aplicado ao algoritmo de Robins e Zelikovsky. 


\section{Considerações finais}

Os algoritmos que apresentamos são simples se comparados com as suas demonstrações de uma razão de aproximação. Na medida do possível, tentamos estabelecer um padrão para a apresentação dos algoritmos e de suas demonstrações. Como dissemos na introdução deste trabalho, o foco principal no estudo desses algoritmos é a demonstração de uma razão de aproximação e não na determinação precisa do tempo de execução dos algoritmos. A tabela abaixo resume, em ordem cronológica, as principais inovações de um algoritmo para o outro e as respectivas razões de aproximação de cada um deles.

\begin{tabular}{||ll|l|r||}
\hline Algoritmo & & Características & Razão \\
\hline MST & 1968 & solução intuitiva & 2 \\
árvores 3-restritas & 1990 & árvores 3-restritas & 1,834 \\
Berman e Ramayer & 1991 & árvores $k$-restritas & 1,734 \\
ganho relativo & 1995 & função ganho relativo & 1,694 \\
pré-processamento & 1996 & florestas de terminais e ganho ponderado & 1,644 \\
Hougardy e Prömel & 1999 & generalização do pré-processamento & 1,598 \\
Robins e Zelikovsky & 2000 & contração de florestas de terminais & 1,55 \\
\hline
\end{tabular}

Razão de aproximação dos algoritmos apresentados.

Embora as dúvidas iniciais sobre esses algoritmos de aproximação baseados em árvores $k$-restritas para o PSG já não existam, no decorrer da elaboração deste trabalho foram surgindo outras que gostaríamos de partilhar com os leitores.

A partir do algoritmo das árvores 3-restritas, não existe comprovação que a razão de aproximação demonstrada é a razão real do algoritmo. Assim pode ser possível melhorar a análise ou pode ser possível que exista uma instância que resulte na razão de aproximação demonstrada.

Será que podemos aplicar os algoritmos para o PSG em variantes do problema encontrando, para estes, razões de aproximação melhores do que as conhecidas na literatura? Um exemplo concreto de problema onde árvores $k$-restritas podem trazer razões de aproximações melhores que a melhor razão conhecida até o momento é o Problema de Steiner com Penalidades (veja detalhes em [20] e no capítulo 4 de [26]). 
Será que a idéia de fazer um pré-processamento nos terminais utilizando como base o algoritmo de Robins e Zelikovsky resulta em uma razão de aproximação menor do que 1,55 ?

Uma outra questão interessante é como os algoritmos descritos nesta dissertação se comportam na prática. Ou seja, quando executados em instâncias construídas aleatoriamente ou obtidas de problemas reais, qual deles obtém a melhor árvore de Steiner? Qual deles consome menos tempo?

Deixamos estas perguntas para leitores interessados em prosseguir no estudo de aproximações para o Problema de Steiner em Grafos e nas suas variantes. 


\section{Referências Bibliográficas}

[1] Y. P. Aneja, An integer linear programming approach to the Steiner Problem in Graphs, Networks 10 (1980), 167-178.

[2] S. Arora, C. Lund, R. Motwani, M. Sudan e M. Szegedy, Proof verification and hardness of approximation problems, Proceedings of $33^{\text {rd }}$ Annual Symposium on Foundations of Computer Science (1992), 14-23.

[3] J. E. Beasley, An algorithm for the Steiner problems in graphs, Networks 14 (1984), 147-159.

[4] J. E. Beasley, An SST algorithm for the Steiner problems in graphs, Networks 19 (1989), 1-16.

[5] P. Berman e M. Karpinski, On some tighter inapproximability results, Eletronic Colloquium on Computational Complexity, TR98-065 (1998).

[6] P. Berman e V. Ramayer, Improved approximations for the Steiner tree problem, Journal of Algorithms 17 (1994), 381-408.

[7] M. Bern e P. Plassmann, The Steiner problems with lengths 1 and 2, Information Processing Letters 32 (1989) 171-176.

[8] J. A. Bondy e U. S. R. Murty, Graph theory with applications, Macmillan, Londres (1976).

[9] A. Borchers e D. Du, The k-Steiner ratio in graphs, SIAM Journal of Computing 26 (1997), 857-869.

[10] T. H. Cormen, C. E. Rivest e R. L. Rivest, Introduction to algorithms, MIT-Press (1998).

[11] R. Courant e H. Robbins, What's mathematics?, Oxford University Press (1977).

[12] R. Diestel, Graph Theory, Springer-Verlag, New York (1997). 
[13] E. W. Dijkstra, A note on two problems in connection with graphs, Numerical Math. 1 (1959), 269-271.

[14] S. E. Dreyfus e R. A. Wagner, The Steiner problem in graphs, Networks 1 (1971), 195-207.

[15] C. E. Ferreira, O problema de Steiner em grafos: uma abordagem poliédrica, Dissertação de Mestrado, IME-USP (1989).

[16] C. E. Ferreira, C. G. Fernandes, F. K. Miyazawa, J. A. R. Soares, J. C. Pina Jr.,K. S. Guimarães, M. H. Carvalho, M. R. Cerioli, P. Feofiloff, R. Dahab e Y. Wakabayashi, Uma introdução sucinta a algoritmos de aproximação, Livro do XXIII Colóquio Brasileiro de Matemática, IMPA (2001).

[17] M. R. Garey e D. S. Johnson, Computers and intractability: A guide to the theory of $\mathcal{N} \mathcal{P}$-completeness, Freeman, San Francisco (1979).

[18] M. R. Garey, R. L. Graham e D. S. Johnson, The complexity of computing Steiner minimal trees, SIAM Journal of Applied Mathematics 32 (1977), 835-859.

[19] E. N. Gilbert e H. O. Pollack, Steiner minimal trees, SIAM Journal of Applied Mathematics 16 (1968) 1-29.

[20] M. X. Goemans e D. P. Williamson, Improved approximation algorithms for maximum cut and satisfiability problems using semidefinite programming, Journal of the ACM 42 (1995), 1115-1145.

[21] C. Gröpl, S. Hougardy, T. Nierhoff e H. J. Prömel, Aproximation algorithms for the Steiner tree problem in graphs, Technical report, Humboldt-Universität zu Berlim (2001).

[22] C. Gröpl, S. Hougardy, T. Nierhoff e H. J. Prömel, Lower bounds for the approximation algorithms for the Steiner tree problem, Technical report, Humboldt-Universität zu Berlim (2001).

[23] M. Grötschel e C. L. Monma, Integer polyhedra arising from certain network design problems with connectivity constraints, Report No. 104, Universität Augsburg (1988).

[24] S. L. Hakimi, Steiner's problem in graphs and its implications, Networks 1 (1971), 113-133.

[25] J. Håstad, Some optimal inapproximability results, Proceedings of the $29^{\text {th }}$ Annual ACM Symposium on the Theory of Computing (1997), 1-10.

[26] D. S. Hochbaum, Approximation Algorithms for $\mathcal{N} \mathcal{P}$-Hard Problems, PWS Publishing (1997). 
[27] S. Hougardy e H. J. Prömel, A 1.598 approximation algorithm for the Steiner problem in graphs, Proceedings of the $10^{\text {th }}$ Annual ACM-SIAM Symposium on Discrete Algorithms (1999), 448-453.

[28] F. K. Hwang, D. S. Richards e P. Winter, The Steiner tree problem, North-Holland, (1992).

[29] A. B. Kahng e G. Robins, On optimal interconnections for VLSI, Kluwer Publishers, (1995).

[30] R. M. Karp, Reducibility among combinatorial problems, Miller e Thatcher (Eds.), Complexity of Computer Computations, Plenum Press, Nova York (1972), 85-103.

[31] M. Karpinski e A. Z. Zelikovsky, New approximation algorithms for the Steiner tree problems, Journal of Combinatorial Optimization 1 (1997), 47-65.

[32] L. Kou, G. Markowsky e L. Berman, A fast algorithm for Steiner trees, Acta Informatica 15 (1981), 141-145.

[33] J. B. Kruskal, On the shortest spanning tree of a graph and the travelling salesman problem, Proceedings Amererican Mathematical Socciety 7 (1956), 48-50.

[34] E. L. Lawler, Combinatorial optimization: networks and matroids, Holt, Rinehart and Winston, New York (1976).

[35] N. Maculan, The Steiner problem in graphs, Annals of Discrete Math. 31 (1987).

[36] K. Mehlhorn, A faster approximation algorithm for the Steiner problem in graphs, Information Processing Letters 27 (1988), 125-128.

[37] M. Minoux, Mathematical programming theory and algorithms, Jonh Wiley and Sons, New York (1986).

[38] C. H. Papadimitriou e K. Steiglitz, Combinatorial optimization, Prentice Hall, Englewood Cliffs, New Jersey (1982).

[39] H. J. Prim, Shortest connection networks and some generalizations, Bell System Technical Journal 36 (1957), 1389-1401.

[40] R. C. Prömel e A. Steger, A new approximation algorithm for the Steiner tree problem with performance ratio $\frac{5}{3}$, Journal of Algorithms 36 (2000), 89-101.

[41] H. Takahashi e A. Matsuyama, An approximate solution for the Steiner problem in graphs, Math. Japonica 24 (1980), 573-577. 
[42] G. Robins e A. Z. Zelikovsky, Improved Steiner tree approximation in graphs, Proceedings of the $11^{\text {th }}$ Annual ACM-SIAM Symposium on Discrete Algorithms (2000), 770-779.

[43] M. L. Shore, L. R. Foulds e P. B. Gibbons, An algorithm for the Steiner problem in graphs, Networks 12 (1982), 323-333.

[44] M. Thimm, On the approximability of the Steiner tree problem, Proceedings of the $26^{\text {th }}$ International Symposium on Mathematical Foundations of Computes Science (2001), Springer LNCS.

[45] V .V. Vazirani, Approximation algorithms, Springer-Verlag, New York (2001).

[46] D. P. Williamson, M. X. Goemans, M. Mihail e V. V. Vazirani, A primal-dual approximation algorithm for generalized Steiner network problem, Combinatorica 15 (1995), 435-454.

[47] P. Winter, Steiner problem in networks: a survey, Networks 17 (1987), 129-167.

[48] A. Z. Zelikovsky, An $\frac{11}{6}$-aproximation algorithm for the network Steiner problem, Algorithmica 9 (1993), 463-470.

[49] A. Z. Zelikovsky, Better approximation bounds for the network and Euclidean Steiner tree problems, Technical report CS-96-06, University of Virginia (1996). 
algoritmo

da árvore geradora, 14

das árvores 3-restritas, 20

de aproximação, 15

de Berman e Ramayer, 32, 34

de Robins e Zelikovsky, 57

do ganho relativo, 41

do pré-processamento, 48

guloso, 17

MST, 15

aproximação

algoritmo de, 15

$\alpha, 15$

probabilística, 17

razão de, 15

aresta

laço, 5

paralela, 5

Arora, 17

árvore, 6

2-restrita, 8

de Steiner, 7

enraizada, 6

geradora, 6

$k$-árvore, 8

$k$-restrita, 8

raiz, 6

Berman, 4, 18

Bern, 17

Bondy, 5

Borchers, 16

busca exaustiva, 14 $c[],$.

$\dot{c}[., .,],$.

caminho, 6

circuito, 6

hamiltoniano, 6

componente, 6

cheio, 8

conectado, 6

conjunto remoção, 9

contração

de florestas de terminais, 54

Cormen, 2

corte, 5

Courant, 3

custo, 6

desigualdade triangular, 6

Diestel, 5

Dreyfus, 13, 14

Du, 16

enumeração explícita, 14

fase

de avaliação, 31

de construção, 34

fecho métrico, 13

Fermat, 2

Ferreira, 12, 14

floresta, 6

de terminais, 45

Foulds, 14

função projeção, 9

$g(.,),$. 
$g_{l}(.,),$.54
$g_{p}(.,),$.46
$g_{r}(.,),$.40
ganho, 12

ponderado, 45

relativo, 40

relativo à perda, 54

Gibbons, 14

Gilber, 15

Goemans, 17

grafo

completo, 5

conexo, 6

definição, 5

euleriano, 6

simples, 5

subgrafo, 5

grau, 5

Hakimi, 13, 14

Håstad, 18

Hougardy, 53

$\operatorname{ind}(., .,),$.

inaproximabilidade, 17

IIndice, 9

Karpinski, 4

Kruskal, 14

Lawler, 14

Leiserson, 2

limite de aproximabilidade, 17

Lund, 17

mst(.,.), 6

Maculan, 2, 3

Mihail, 17

Moore, 15

Motwani, 17

Murty, 5

$\mathcal{N P}$ completo, 14

difícil, 2

otimização combinatória, 2

passeio, 6

Plassman, 17

Pollack, 15

Prömel, 53

Prim, 14

primal-dual, 17

problema

da cobertura exata, 14

de decisão, 14

Problema de Steiner, 3 complexidade computacional, 14 definição, 13

instância, 14

soluções, 14

programação

dinâmica, 14

inteira, 14

projeção, 9

da contração, 55

$Q(.,),$.

$r_{k}, 16$

Ramayer, 4

redução, 6

restrição, 7

Rivest, 2

Robbins, 3

Robins, 54

$\operatorname{smt}(., .,),$.

$\operatorname{smt}_{k}(., .,),$.

separa, 5

separador, 8

Shore, 14

Steiner, Jacob, 3

subgrafo

definição, 5 
gerador, 5

induzido, 5

Sud an, 17

Szegedy, 17

Thimm, 18

trilha, 6

euleriana, 6

trip 1 a, 20

$V S(-), 8$

$\operatorname{VT}(),$.

Vazirani, 15, 17

vértice

adjacente, 5

de Steiner, 7

extremos, 5

terminais, 7

Wagner, 13, 14

Williamson, 17

Zelikovsky, 4, 20 